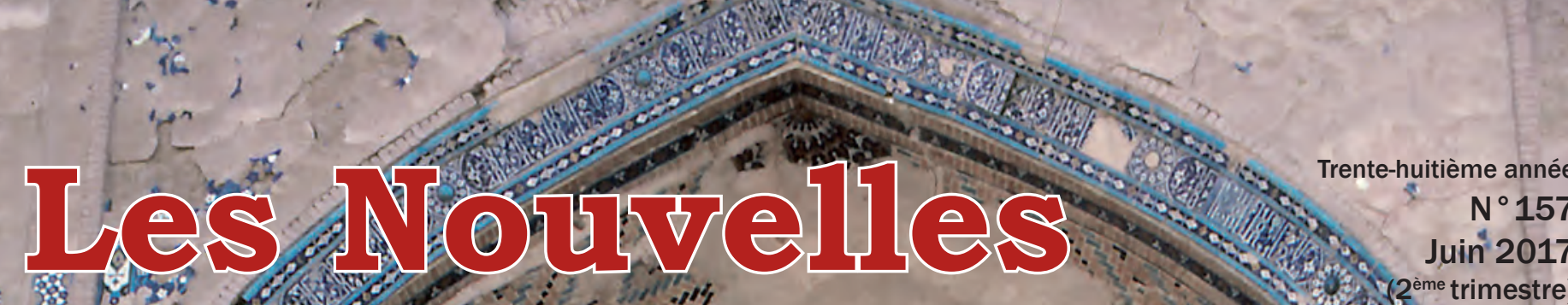

屋

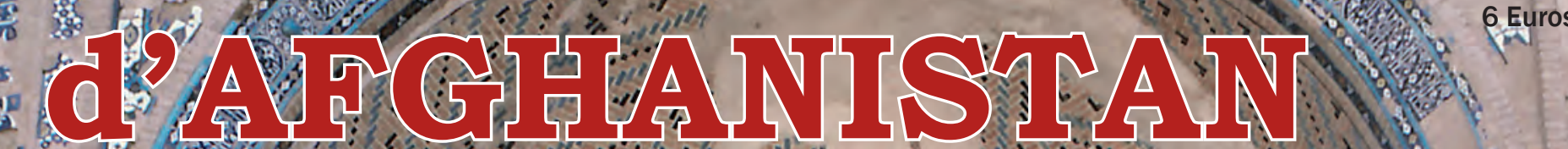

Fis

का

:

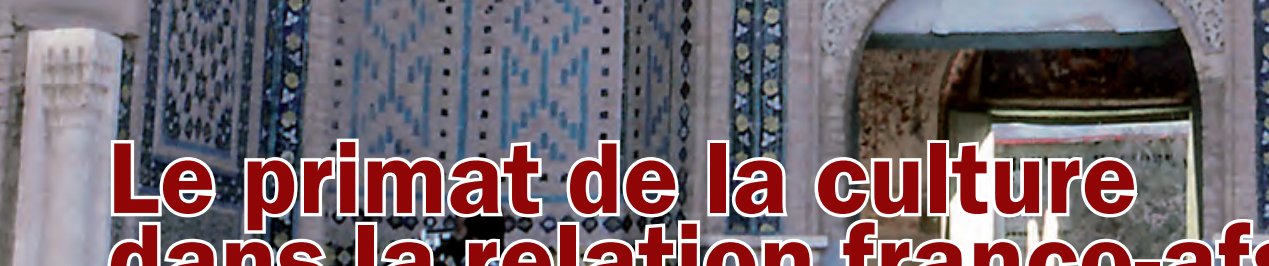
dans larelation franco-afghaine

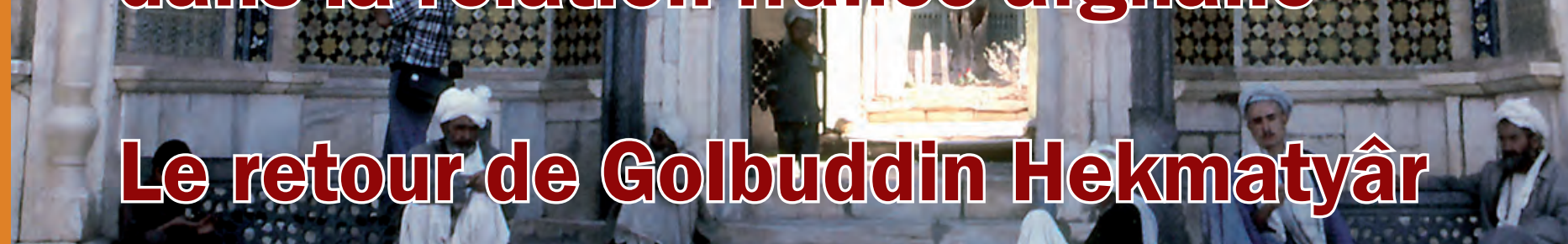

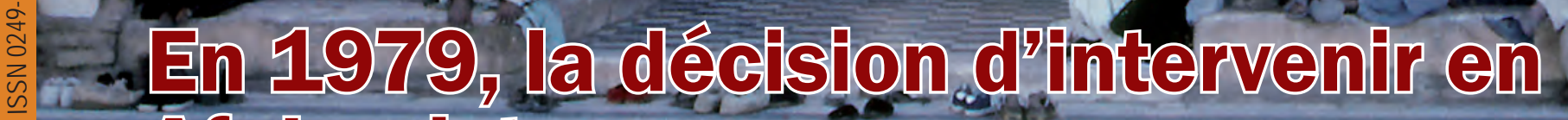
Alfighanistan 


\section{Violence et culture}

I apparaîtra peut-être paradoxal que ce numéro donne la part belle à des questions culturelles ou historiques alors que la violence s'accroît en Afghanistan. Le dernier attentat en plein Kaboul, d'une intensité extrême (le cratère creusé par l'explosion d'un camion bourré d'une tonne et demie d'explosifs mesurait quatre mètres de profondeur), a secoué autant les esprits que les habitations qui ont tremblé jusqu'à trois kilomètres à la ronde. Les auteurs de l'attentat ont ainsi réussi au-delà de ce qu'ils pouvaient espérer. Ils ont non seulement traumatisé la population mais ils ont également créé des divisions en son sein et par là-même fait ressortir encore davantage la fragilité du pouvoir. «A tous les coups que nous portons, nous gagnons » doivent penser les cyniques concepteurs de ces attaques aveugles.

Dans le même temps, les tractations diplomatiques se succèdent. Chaque puissance régionale essaie de se placer pour pouvoir jouer un rôle dans l'Afghanistan de demain. Les Chinois, les Russes, les Indiens, les Pakistanais bien sûr, les Iraniens, tous veulent participer à ce nouveau grand jeu. Ce nouveau grand double jeu, allant parfois jusqu'à armer en sous-main les insurgés. Les Américains quant à eux semblent hésiter entre la tentation de se désengager et celle de renforcer leurs effectifs. L'absence de politique claire, la maladresse des interventions effectuées, l'ambiguiité de la diplomatie des Américains, le soutien apporté apparemment aux Tâlebân par leur allié pakistanais, tout cela entraîne dans la population afghane un scepticisme prononcé à l'égard de la pureté des intentions américaines. Quant à l'Europe, elle a disparu des écrans, à l'exception de l'Allemagne.

Dans ces conditions, évoquer les liens culturels entre la France et l'Afghanistan, revenir sur des événements historiques déjà anciens n'est-il pas un peu décalé ? Nous sommes pourtant très honorés de publier les contributions d'un haut niveau que nous avons rassemblées dans ce numéro. Outre leur intérêt propre, elles permettent de toucher à l'essentiel.

Le questionnaire envoyé par AFRANE aux candidats à l'élection présidentielle n'a obtenu aucune réponse des « grands » candidats. Cela pourrait laisser croire que l'Afghanistan n'intéresse plus nos politiques. Pourtant, livres, documentaires, films sur l'Afghanistan continuent de paraître, montrant que le lien que l'on pourrait qualifier de spirituel entre nos deux pays continue d'être fort. De même l'action des ONG françaises reste importante. II convient donc de redoubler d'efforts pour convaincre les responsables politiques de l'importance qu'a l'Afghanistan dans la construction de la paix mondiale. Beaucoup de Français perçoivent la nécessité d'aider les Afghans à relever les multiples défis humains, culturels et sociaux devant lesquels ils se trouvent. Un leader afghan a évoqué récemment le risque que l'Afghanistan devienne une nouvelle Syrie. Nous devons avoir conscience des dangers que représenterait, y compris pour la France, un nouvel embrasement afghan.

\section{Etienne GILLE}

18 juin 2017

Site internet : www.afrane.org

ACTUALITÉ

Le retour d'Hekmatyâr par Valérie DOCHER

. Le jeu diplomatique de la Russie et de l'Iran

par Chahir ZAHINE

TÉMOIGNAGE

Trente images pour trois années par Jean-Michel MARLAUD

HISTOIRE

. Mohammad Moussa Chafiq par Zia FARHANG

- L'intervention militaire soviétique vue de Moscou

par Gilles ROSSIGNOL

CULTURE

Le primat de la culture dans la relation franco-afghane par Régis KOETSCHET

SOLIDARITÉ

Afghanistan Info 1980-2017

par Micheline CENTLIVRES

DERNIERES NOUVELLES

Chronologie, brèves, bibliographie

Photo de couverture: Porche d'entrée du mausolée d'Ansari à Gozargâh, près de Hérat, 1972. (Jean-Michel Marlaud l'évoque dans son article en $p$. 9). Photo Véra Marigo.

Les Nouvelles d'Afghanistan bénéficient d'une aide financière de l'ambassade de France en Afghanistan Adresse E-mail

afrane.paris@gmail.com

Les Nouvelles d'Afghanistan 16, passage de la Main d'Or -75011 Paris 


\title{
Le retour d'Hekmatyâr
}

\author{
par Valérie DOCHER*
}

\begin{abstract}
Golbuddin Hekmatyâr est un personnage pour le moins controversé de la scène politique afghane. Dans un précédent numéro ${ }^{1}$ nous avons rappelé l'histoire de son parti, le Hezb-e Islâmi. Agé de 70 ans, responsable avec d'autres du bombardement de Kaboul dans les années de la guerre civile, suspecté d'avoir commandité des assassinats politiques, classé terroriste par les instances internationales, G. Hekmatyâr a choisi finalement de négocier avec le gouvernement sa réintégration dans un jeu politique pacifique. Ralliement sincère ou mesure tactique?
\end{abstract}

Lorsque je suis arrivée en 2010 à Kaboul, celui qui allait devenir mon mentor afghan, un médecin respectable me disait : « le seul qui peut ramener la paix dans ce pays c’est Hekmatyâr ».

La maladie l'a emporté il y a quelques mois et il n'aura pas vu le retour officiel d'Hekmatyâr dans le Laghmân, dans le Nangarhâr puis à Kaboul, lors d'un des plus grands rassemblements connus dans le Ghâzi Stadium, ce 4 mai 2017. Des personnes étaient venues de toutes les provinces pour revoir Hekmatyâr à Kaboul. Les chiffres varient en fonction des sources et ne sont pas assez fiables pour donner une idée précise du nombre mais le Ghâzi Stadium était rempli de drapeaux verts. Ce même Stadium qui aura vu passer les concerts de Gougouch², les Tâlebân et les exécutions. Il faudra un jour écrire les mémoires de ce stade.

Hekmatyâr a rassemblé ses partisans qui s’étaient souvent tenus dans l'ombre pendant des années attendant son grand retour avec impatience. Des jeunes, des moins jeunes et quelques centaines de femmes étaient présents. Hekmatyâr était venu avec sa femme et sa fille, Amin Karim, le porte-parole de son parti (le Hezbe Islâmi) avec la sienne. Est-ce un tournant dans la vi-

* Secrétaire Générale de MRCA. A séjourné en Afghanistan de 2010 à 2015 et s'y est rendu depuis tous les trimestres.

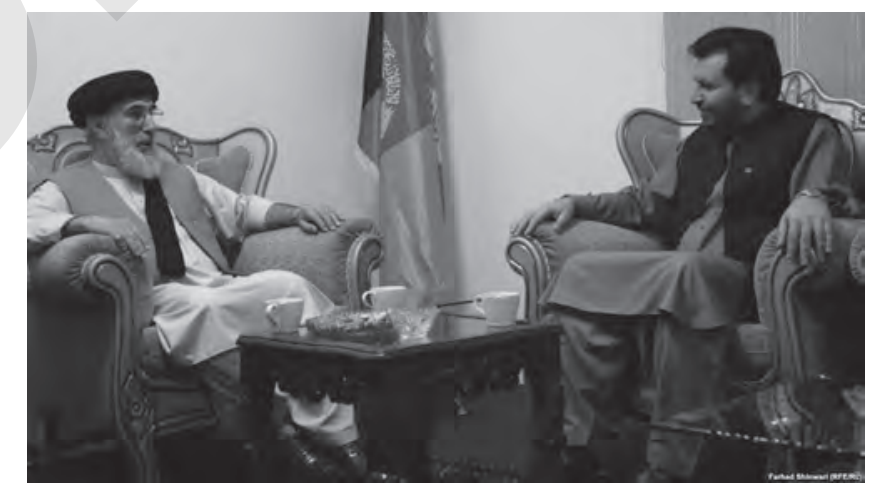

Golbuddin Hekmatyâr reçu par le gouverneur du Laghmân. Photo DR

sibilité des femmes dans le Hezb-e Islâmi Afghanistan (HIA) ou un clin d'œil à certains dirigeants afghans dont les familles sont à Dubaï ou en Inde ? Lors des réceptions officielles, la femme d'Hekmatyâr était présente comme pour indiquer qu'il était venu en famille et pour rester.

Pendant que des milliers d'Afghans accueillaient Hekmatyâr, des milliers d'autres voyaient dans cet accord un premier pas pour la paix dans le pays et d'autres enfin, demandaient justice pour les crimes commis par Hekmatyâr dans le passé. Avec l'accord de paix signé entre le gouvernement d'union nationale et le HIA, 


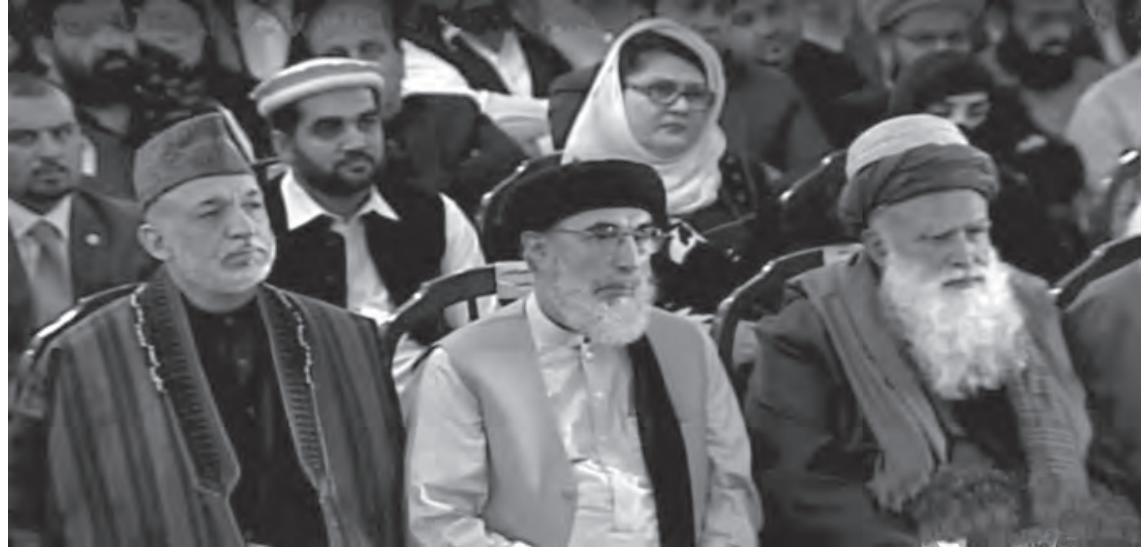

Hekmatyâr Golbuddin, entre Hâmed Karzaï et Sayyâf au Palais présidentiel de Kaboul. Photo DR

Hekmatyâr enrichit la longue liste des chefs de guerre réhabilités sans être passés devant la justice. Les prisonniers du HIA ont également été libérés.

\section{L'accord de paix}

L'accord de paix qui a permis le retour d'Hekmatyâr est le résultat d'années de discussions entre le gouvernement, les États-Unis, l’Union Européenne et les émissaires d'Hekmatyâr. Les premiers contacts datent de mars 2010, avec, entre autres, Karzaï, Rabbani et Sayyâf. Ces contacts irréguliers étaient maintenus par l'intermédiaire de courriers officiels et d'envois d'émissaires et se sont poursuivis jusqu'en 2012 avec une rencontre majeure pour le HIA avec des généraux américains. La poursuite des contacts a permis au HIA de soutenir officiellement des candidats aux élections de 2014, se repositionnant ainsi dans une démarche plus officielle et politique. Un accord de paix était prêt à être signé en 2014 mais le gouvernement à deux têtes souhaité par les Américains a remis en cause cet accord potentiel. En février 2015, sur la base des documents du premier accord, le président Achraf Ghani renvoie

Kaboul. Arrivée officielle au Palais présidentiel de Golbuddin Hekmatyâr, entouré de Hâmed Karzaï, Achraf Ghani et Sayyâf. Photo DR

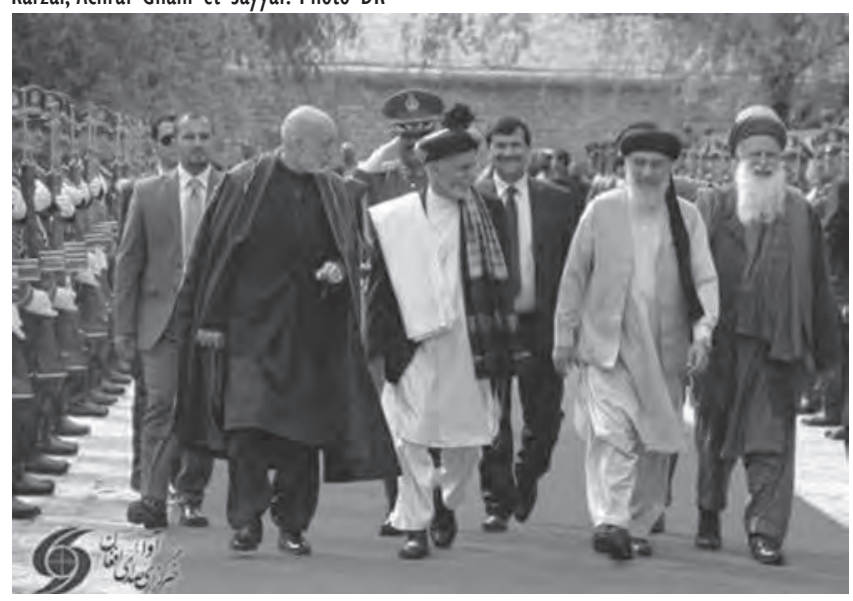

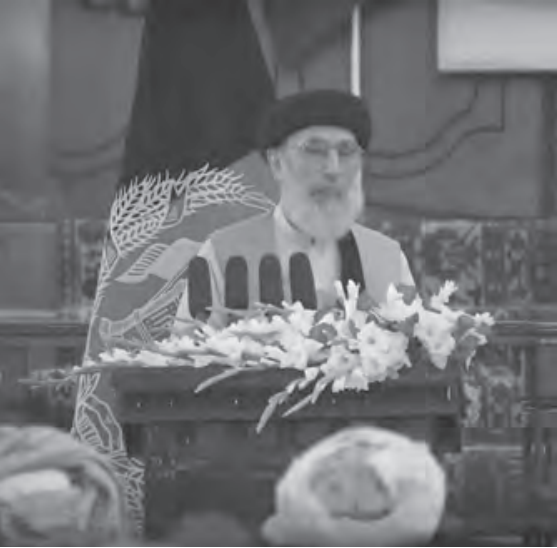

Hekmatyâr Golbuddin, premier discours officiel au Palais présidentiel de Kaboul. Photo DR les négociations devant le Haut Conseil de la Paix. De nouveaux longs mois de négociations s'entament alors avec en fond, le parti politique Djamiat, plutôt défavorable à toute avancée. Soixante et une versions de cet accord seront faites et les articles rediscutés inlassablement de part et d'autre, jusqu’à sa signature le 22 septembre 2016.

Alors qu'Amin Karim est le porte-parole du HIA dans les discussions, les ambassades occidentales n'apportent que peu de soutien au processus de paix, restant en retrait et sceptiques : elles considèrent que le HIA n’a qu'une faible présence en province, un faible poids politique et n'est pas en mesure de changer la donne dans le pays. De leur côté, les Américains ne veulent pas apparaître en première ligne, et l'ambassadeur américain ne souhaite pas de rencontre directe avec les représentants du HIA. La France quant à elle, reçoit le négociateur officiel, apprécie la condamnation officielle faite par le HIA concernant les attentats sur l'Institut français d'Afghanistan. L'ambassadeur de l'Union Européenne apporte son soutien politique à cet accord, mais c'est surtout la volonté du président Ghani de signer qui a permis l'avancée des négociations jusqu'à sa signature.

Ainsi, le meilleur allié du HIA dans ces négociations aura sans doute été le président Ghani, soucieux de montrer des avancées dans le processus de paix et de garantir un partenariat avec ce groupe capable de revenir comme une force politique dans le pays. Les Américains, sans doute pour montrer enfin un accord de paix dans un pays où ils sont présents militairement depuis quinze ans, ont aussi soutenu l'accord. La force de l'exemple pour amener les Tâlebân à la table des négociations est une des stratégies poursuivies.

\section{L'organisation du retour}

La signature de l'accord est une victoire pour le HIA. Ses responsables sont invités dans des pays étrangers, pour rencontrer les gouvernements et d'anciens 


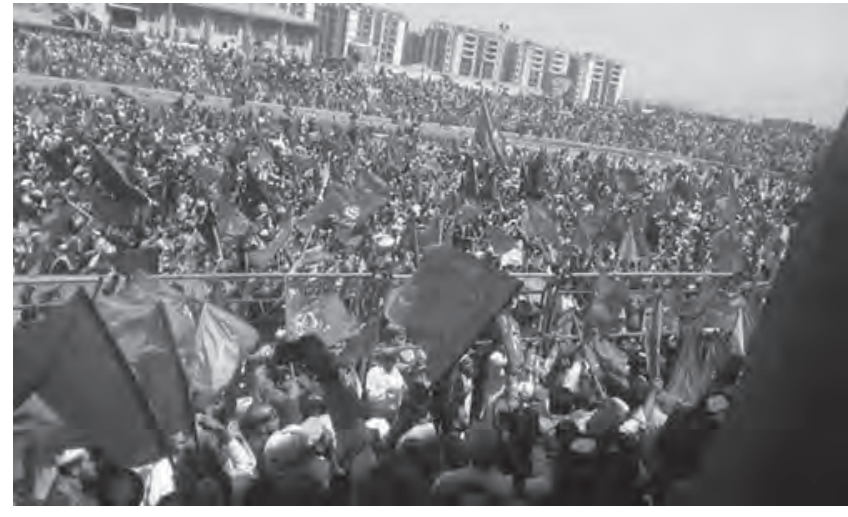

Kaboul, Ghâzi Stadium. «Des personnes étaient venues de toutes les provinces pour revoir Hekmatyân). Photo DR

groupes terroristes ayant réintégré la vie politique. Réunions auxquelles ils ne peuvent pas toujours participer car la tâche en interne en Afghanistan est très lourde et ils sont peu nombreux à pouvoir tenir un rôle politique international. Dès la date de la signature de l'accord, un des points majeurs pour le HIA est de préparer le retour en Afghanistan d'Hekmatyâr (resté " caché » quelque part au Pakistan) quand les mesures de sécurité seront assurées. Un groupe de travail interne est constitué en 2017 pour préparer non seulement son retour à Kaboul mais aussi la communication autour de « l'évènement », avec le souhait de faire une démonstration de présence et de marquer les esprits par ce retour. Un mois de travail pour ce groupe pour préparer l’arrivée, informer les équipes en interne sans ébruiter trop d'informations, en préparant un passage par le Laghmân, puis le Nangarhâr et l'arrivée à Kaboul. Les modalités initiales du retour ne sont pas acceptées par le président Ghani et la date du retour est repoussée de quelques semaines. Ils auront eu au final quelques jours pour rassembler la foule dans le stadium.

\section{Le HIA en 2015}

Depuis des années, les membres du HIA ne se revendiquent plus explicitement de leur parti, même s'ils restent présents et organisés dans les provinces. Le HIA est présent officieusement au Parlement avec 34 parlementaires (33 aujourd'hui, un d'entre eux ayant été tué) et plusieurs gouverneurs en poste issus de ce groupe. Les ministres liés au HIA sont au nombre de 3, plus un se revendiquant du HIA en fonction du jour, du sujet et de l'intérêt. Ils représentent une force politique capable de jouer un rôle dans les nominations et dans les décisions politiques internes. La plupart de ses partisans sont éduqués. Parmi eux, de nombreux ingénieurs et médecins en poste dans le pays. Ils sont également près de 2000 familles à l'étranger, en Allemagne, en Angleterre, aux Etats-Unis et en France, prêtes en prin- cipe à revenir si les conditions économiques proposées en Afghanistan le leur permettent, afin de prendre des postes à responsabilité.

Le groupe est lui-même éclaté entre plusieurs branches internes faites de conservateurs et de modérés. La communication est devenue un axe stratégique du HIA. Avec un porte-parole connaissant parfaitement les codes afghans et occidentaux, de par son installation en Europe depuis quarante ans, le HIA a une stratégie de communication modérée, intégrant le droit des femmes. Des groupes de femmes sont créés au sein du parti avec des rencontres régulières. Trois femmes participent maintenant au conseil de décision élargi. Elles rencontrent des délégations occidentales. Il sera important de regarder de près leurs actions et de comprendre leurs rôles dans le parti. Il reste encore que le conseil suprême, constitué de sept personnes, est uniquement tenu par des hommes. Il sera intéressant de voir si la branche conservatrice du parti finit par accepter l'intégration des femmes dans l'organe central de décision et si ce travail actuel avec les femmes perdure et est efficace.

Lors d'une de mes rencontres avec une femme politique afghane, elle confiait que les discussions avec les membres du HIA étaient plus aisées car plusieurs de leurs membres avaient vécu en Europe et avaient intégré une vision différente du rôle des femmes. Elle n'était bien sûr pas en mesure de dire si cela pourrait s’inscrire dans la durée.

\section{Et maintenant}

Hekmatyâr est à Kaboul et veut renforcer son rôle de faiseur de paix en entamant des discussions avec ses « frères » Tâlebân. Quelles sont les avancées connues ?

Deux stratégies ont été mises en place :

- Des commandants tâlebân ont été approchés directement pour se joindre au HIA. Une centaine d'entre eux seraient prêts à le faire selon les dire du HIA ; ce sont pour la plupart des anciens du parti qu'un ralliement direct au gouvernement ne semble pas séduire : passer par le HIA pourrait être une option, option qui renforcerait aussi les rangs du HIA.

- Hekmatyâr rencontre les Tâlebân de Doha pour des négociations. Mais ces rencontres sont-elles représentatives du pouvoir central tâleb ? Tous ne sont pas prêts à rentrer dans des négociations de paix. Le processus est et sera lent.

Actuellement, Hekmatyâr rencontre également les officiels afghans et internationaux, les Nations unies, et travaille sur son positionnement d'homme politique qui compte. Sa proposition en cours est de mettre autour d'une même table les Afghans importants de la vie politique actuelle afghane, de Sayyâf à Ghani en passant par Qânouni pour parler d'une seule voix dans 
les accords de paix avec les Tâlebân. Mais cette grande consultation et unification des différents partis fait craindre à certains, proches du pouvoir, que se mette ainsi en place dans les prochains mois une coalition contre le gouvernement.

Le HIA ne demande à ce jour aucun rôle officiel dans le gouvernement bien que plusieurs postes lui aient été proposés. L’article 7 de l'accord les autorise à se présenter à toutes les élections : ils présenteront donc des candidats, peut-être pas à la prochaine élection présidentielle car trop peu de choses auront pu être achevées d'ici là pour accéder au pouvoir, mais ils seront suffisamment importants pour représenter une force politique dans les négociations pour constituer le gouvernement.

L'autre interrogation légitime reste le rôle d'Hekmatyâr de par le soutien dont il a bénéficié au Pakistan ? Y aurait-il un souhait de déstabilisation politique du gouvernement afghan par l'intervention du Pakistan et l'intermédiaire d’Hekmatyâr ? Cela rejoint donc la question posée plus haut sur cette stratégie de coalition multi-partis et son utilisation à terme.

\section{Les réfugiés de Shamshato}

Le gouvernement pakistanais pousse les réfugiés afghans à rentrer dans leur pays. Les camps gérés par le HIA à Peshawar sont concernés. Shamshato est le plus grand d'entre eux et est connu pour abriter depuis de nombreuses années le quartier général du HIA. Le HIA tente de négocier avec le gouvernement afghan des terres, la construction de villes en périphérie de Kaboul pour permettre aux réfugiés de rentrer dans de bonnes conditions.

A ce jour, il y a peu d'avancées malgré les promesses initiales. Les réfugiés représentent pour le HIA une force de soutien et des adeptes importants et le parti tente de trouver des solutions pour leur assurer un retour correct.

\section{Le rôle de la France}

Comme noté plus haut, la France s’intéresse à ces accords de paix, sans pour autant jouer un rôle majeur. Le représentant du HIA représente-t-il l'ensemble des branches du HIA ? Ces négociations seront elle remises en cause ? La France est présente, avec des discussions diplomatiques plus que des interventions politiques. Pas de prise de position ferme et officielle sur le sujet, aucune reconnaissance jusqu'à la signature de l'accord. Les forces françaises présentes en Kapisa jusqu'à la fin 2014 étaient en conflit direct avec les combattants du HIA à Tagâb ou en Alasây. Les Français étaient au combat et la bataille d'Ouzbin en août 2008 avec les soldats français morts au combat est encore très présente dans les mémoires. Il est difficile politiquement de soutenir un groupe qui a participé, avec les Tâlebân, à la mort de dix soldats français et a blessé 21 autres quelques années plus tôt.

A ce jour, les questions centrales restent donc de savoir

1) si la nouvelle stratégie de positionnement du HIA sera pérenne et si les modérés qui sont actuellement en position forte de par les succès des négociations et du retour d'Hekmatyâr auront les moyens financiers de continuer à soutenir leurs approches et propositions,

2) si cette nouvelle approche est un nouveau positionnement ou seulement une stratégie pour revenir dans la vie politique.

1- Les Nouvelles d'Afghanistan, $n^{\circ} 153$, juin 2016.

2- Célèbre chanteuse iranienne.

\section{Les Nouvelles d'Afghanistan}

La revue LES NOUVELLES D'AFGHANISTAN est une revue trimestrielle éditée par AFRANE (Amitié Franco-Afghane). Les opinions émises dans les articles n'engagent que leurs auteurs. Titres et soustitres sont de la responsabilité de la rédaction.

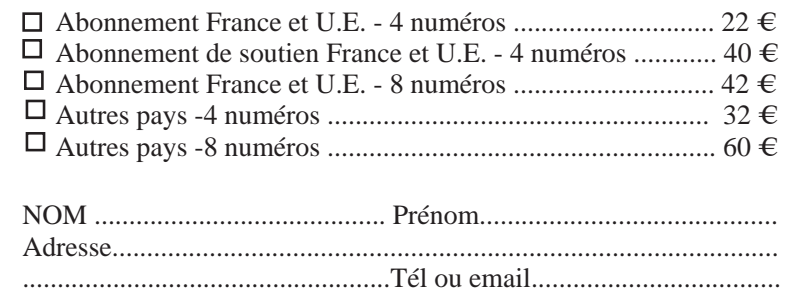

\section{Afrane}

Permanence: 16 passage de la Main d’Or -75011 Paris Tel. : (33) 01.43.55.63.50

L'association Amitié Franco-Afghane (Afrane) a été fondée au début de 1980, en réponse à l'occupation militaire de l'Afghanistan par les Soviétiques. Organisme d'aide humanitaire, Afrane ne souhaite qu'aider les Afghans et ne se situe dans la mouvance d'aucun pati politique.

Elle soutient à présent prinicpalement des projets éducatifs.

$\square$ Adhésion avec abonnement à la revue

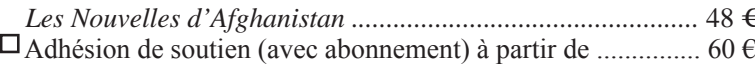

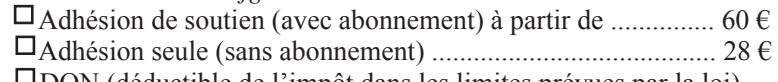

$\square$ DON (déductible de l'impôt dans les limites prévues par la loi)

(un reçu sera adressé sur demande) ............................................ $€$

NOM .

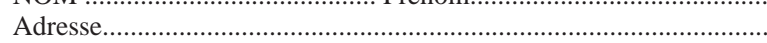

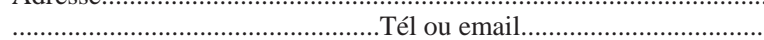




\title{
Le jeu diplomatique de la Russie et de l'Iran
}

\author{
par Chahir ZAHINE*
}

\begin{abstract}
Des rumeurs de plus en plus fortes font état de soutiens russes aux Tâlebân. De son côté l'Iran aurait aussi plusieurs fers au feu. Chahir Zahin, journaliste, fait le point des différentes informations portant sur les diplomaties russe et iranienne et propose son analyse.
\end{abstract}

Le 22 et le 23 mai 2017 l'Ambassade de Russie à Kaboul a envoyé deux communiqués aux médias afghans et autres pour démentir les dires de différents éléments du gouvernement afghan concernant la présence de conseillers russes et le support militaire de ce pays aux cotés des Tâlebân. En effet, les jours et les semaines précédant ces deux communiqués, différents membres des forces de sécurité afghanes et de l'administration (les commandants de police des provinces d'Ourouzgân, de Baghlân et du Helmand, et les gouverneurs de Balkh et de Koundouz) ainsi que des parlementaires avaient accusé la Russie d'apporter un soutien en argent, en armement et en logistique aux Tâlebân.

La Russie par le passé avait reconnu qu’elle était en contact avec les Tâlebân pour aider le processus de paix en Afghanistan. Mais depuis quelques mois des membres du gouvernement afghan ainsi que des militaires américains ont mentionné la Russie parmi les soutiens actifs des Tâlebân. En mai 2016, quand le leader des Tâlebân, Moullah Akhtar Mohammad Mansour, a été tué dans une attaque de drone américain en territoire pakistanais, il était supposé revenir d’un entretien avec les Russes en territoire iranien.

\section{Un axe Russie-Iran-Tâlebân?}

Le soutien des Iraniens aux Tâlebân est beaucoup plus ancien. C'est en septembre 2008 que pour la pre-

* Responsable d'organes de presse. mière fois une journaliste de la BBC, Kate Clark, avait fait un reportage où les Tâlebân parlaient ouvertement du soutien reçu de l'Iran. L'armée afghane et les forces de l'OTAN étaient alors victimes de mines antichars très puissantes et presque indétectables. Le reportage de la BBC faisait état de ces mines d'origine iranienne et de leur puissance admirée par les Tâlebân ${ }^{1}$.

Mais, c'est seulement depuis quelque temps qu'on évoque l'axe Russie, Iran, Tâlebân. En effet, aussi bien les officiels afghans que les généraux américains commencent à, explicitement, impliquer les Iraniens et les Russes dans l'approvisionnement en armes et munitions des Tâlebân. Le 23 mars 2017, le général américain, Curtis Scaparrotti, commandant suprême des Forces Alliées de l'OTAN en Europe, a averti que la Russie a approfondi ses liens avec les Tâlebân. En février dernier quand le général Scaparrotti a témoigné devant le comité des forces armées du Sénat américain il a déclaré : «j’ai constaté dernièrement que la Russie a augmenté son influence en termes de coopération et même en approvisionnement auprès des Tâlebân ". Dans la même veine le Commandant américain en Afghanistan, le géneral Nicholson a dit que la Russie et l'Iran soutenaient les Tâlebân pour saper la mission de l'OTAN et des Etats Unis en Afghanistan.

\section{De nouvelles alliances}

Depuis quelques années nous sommes témoins d'une redéfinition des alliances autour de l'Afghanistan. Le Pakistan qui était, lors de la guerre froide et même 
après, un allié des Etats-Unis et de l’Arabie Saoudite est en train de se rapprocher de la Russie et de l'Iran. L'Inde qui était proche de Moscou est de plus en plus en coordination avec les Américains.

La relation très tendue et conflictuelle entre la Russie et le Pakistan est en train de changer en une coopération stable. Ce changement s'est opéré en une dizaine d'années, depuis la première visite, en 2003, du Président Pervez Musharraf à Moscou, puis la visite en 2007 du Premier Ministre russe, Mikhael Fradkov, au Pakistan et finalement la levée par la Russie, en 2014, de l'embargo sur les achats d'armements russes par le Pakistan et la livraison en conséquence d’hélicoptères MI35 au Pakistan. Depuis ce temps les deux pays ont fait des manœuvres militaires maritimes conjointes en 2014 et 2015 et viennent d'annoncer d'autres manœuvres conjointes pour 2017.

N'oublions pas que le Pakistan a toujours eu une relation privilégiée avec la Chine qui n’a pas arrêté de soutenir le Pakistan y compris dans le domaine nucléaire (le premier test nucléaire du Pakistan le 28/05/1998 n’a été possible que grâce à l'aide et la technologie chinoises.

Depuis les sanctions internationales contre la Russie, après l'annexion de la Crimée, la Russie a tenté des ouvertures vers la Chine qui de son côté trouve une aubaine, parce qu'en aidant le Pakistan, elle peut consolider sa suprématie régionale et son assise internationale. Du coup un axe Russie, Pakistan, Chine se profile qui signifie la redéfinition des alliances régionales.

\section{Le jeu iranien}

Depuis le départ des Soviétiques de l'Afghanistan (15/02/1989), l'Iran et le Pakistan ont souvent collaboré pour poursuivre des intérêts concordants. Durant les années de guerre civile (1992-1996) et le règne des Tâlebân (1996-2001), plusieurs fois les services iraniens et pakistanais ont travaillé ensemble pour poursuivre leurs agendas. Les plus fameuses de ces coopérations sont les différentes réunions de « paix », vers la fin des années Tâlebân, à Chypre², dont les Iraniens étaient les financeurs et les Pakistanais les organisateurs. Cette coordination a eu des hauts et des bas, mais, elle n’a jamais cessé.

Depuis le départ, fin 2014, de la plupart des forces internationales de sécurité de l'Afghanistan, il y a une nouvelle occasion pour ces voisins et les pouvoirs régionaux de vouloir s’immiscer davantage dans les affaires de ce pays. Pour l'instant le dicton ; «l'ennemi de mon ennemi est mon ami » prévaut dans leurs démarches face à l'Afghanistan. L'Iran et la Russie n'étant pas dans les meilleurs termes avec les Etats Unis/OTAN, du coup ils se mettent ensemble pour saboter les efforts de ces derniers en soutenant les Tâlebân. Les Iraniens et les Russes ont tous les deux utilisé une même raison officielle, pour justifier leurs relations avec les Tâlebân en la justifiant comme une nécessité pour combattre le soi-disant Etat Islamique. Mais en réalité les enjeux sont beaucoup plus importants et complexes

L'Iran veut profiter des divisions au sein de l'OTAN (Trump/les Européens) et au sein du gouvernement afghan (Ghani/Abdullah) pour se placer en meilleure posture et surtout pour pouvoir négocier ultérieurement au niveau de la région comme un joueur important. Les nouvelles tournées de négociations sur son deal nucléaire vont revenir rapidement. Par ailleurs dans son bras de fer avec l'Arabie Saoudite sur le Yémen, il veut montrer sa capacité à se battre sur plusieurs fronts, surtout à des coûts dérisoires.

La Russie joue sur plusieurs tableaux ; avoir une monnaie d'échange dans son bras de fer sur l'Ukraine, réorganiser ses alliances régionales, mais aussi saper le moral des Occidentaux en Afghanistan. Pour la Russie et l'Iran les Tâlebân représentent un instrument facile pour contenir l'expansion de Daech, pour mettre la pression sur le gouvernement afghan et ses alliées US/ OTAN et pour étendre leur influence régionale.

Pour les Tâlebân, qui ont traditionnellement eu comme base arrière le Pakistan où l'on suppose que la majorité de leurs leaders résident toujours, ce soutien international est une bonne affaire. Car en plus de sources supplémentaires d'approvisionnement, ils ont surtout acquis une reconnaissance extérieure qui semble légitimer leur cause et leur combat. Déjà depuis de longues années, au niveau local, dans une bonne partie des villages afghans, les Tâlebân font régner l’ordre et la loi quand l'Etat afghan s'en montre incapable. Maintenant, c'est pour les pouvoirs régionaux que les Tâlebân peuvent jouer le rôle d'alternative qui assure la sécurité, quand l'Etat afghan ne peut la délivrer. En effet la Russie et l'Iran reconnaissent avoir des contacts avec les Tâlebân pour s’assurer que l'expansion du soidisant Etat Islamique est contenue...

Dans ce contexte l'Afghanistan se retrouve au milieu d'un bras de fer continuel dont les joueurs et les mains changent mais c'est toujours sur le sol afghan que sont les dégâts et c'est surtout les Afghans qui sont les principales victimes.

L'emplacement géographique de l'Afghanistan en tant que couloir entre l'Asie Centrale et le sous-continent aussi bien qu'entre le Moyen-Orient et le souscontinent ne peut être ignoré. Cette région, dans l'ensemble, ne peut prospérer tant que l'Afghanistan n'est pas un lieu de passage sûr. La situation de l'Afghanistan ne peut changer que s'il y a un consensus régional et international pour garantir la paix dans ce pays et permettre à la région de prospérer.

1- http://news.bbc.co.uk/2/hi/south_asia/7623496.stm

2- Le processus de Chypre était initié par des anciens du gouvernement de Kaboul, aussi bien que par des modjahedin proches des services iraniens et pakistanais qui voulaient créer un groupe pour faire contrepoids au processus de Rome qui était centré autour de l’ancien Roi Zâher Châh. 


\title{
Trente images pour trois années
}

\author{
par Jean-Michel MARLAUD*
}

\begin{abstract}
Monsieur Jean-Michel Marlaud a accepté de communiquer aux lecteurs des Nouvelles d'Afghanistan les impressions, souvenirs essentiellement visuels, que lui a laissé son séjour en Afghanistan. Ces images, à la fois fortes et simples, disent bien des choses de la situation de ce pays aujourd'hui.
\end{abstract}

Lors de son Assemblée Générale AFRANE m’a proposé de partager les images qui me restent de mon séjour de trois ans en Afghanistan. J'ai joué le jeu, en les mettant noir sur blanc au fur et à mesure qu'elles me venaient à l'esprit, sans chercher à leur donner un sens. J'ai néanmoins vite constaté qu'elles pouvaient être réunies sous quatre thèmes et que le choix de leur ordre de présentation n’était pas neutre.

La rencontre à AFRANE au cours de laquelle j'ai partagé mes souvenirs s'est tenue le 21 avril, au lendemain de l'attaque meurtrière de Mazar-e Charif. La première image qui s'est présentée à moi était donc celle de la base militaire où s’était déroulée cette attaque et que j’avais visitée lors de mon premier séjour dans cette ville. Après un hôpital, c'est une assemblée d'hommes désarmés et en prière qui a été la cible des terroristes. Au-delà même de la barbarie de tels actes, quel peut être le modèle de société que proposent leurs auteurs ?

Nous sommes tous influencés par notre passé, et plus encore lorsqu'il s'agit de l'Afghanistan, pays qui en appelle à notre imaginaire. J'y suis arrivé nourri de mes souvenirs d'adolescent : les Cavaliers de Kessel, l'autochenille Citroën devant les Bouddhas de Bâmyân pendant la Croisière Jaune. Rien d'étonnant donc si mes premières évocations sont liées à l'Afghanistan millénaire.

\footnotetext{
* Diplomate, ancien ambassadeur de France en Afghanistan de 2013 à 2016.
}

\section{Hauts lieux}

Je commencerai donc par une visite à Balkh avec le directeur de la DAFA. Après avoir vu les chantiers de fouilles et les monuments de l'oasis, Philippe Marquis nous a emmenés à sa lisière pour nous faire grimper jusqu'à un faux plat d'où la vue était à couper le souffle. Toutefois, il ne fallait pas regarder au loin mais à nos pieds, où reposait une pierre à vrai dire peu spectaculaire. Une pierre ? Non : un autel du feu, dont la taille exceptionnelle, combinée à l'étude des textes anciens, permettait de penser qu'elle marquait l'emplacement du principal temple zoroastrien de l'époque... autrement dit, le lieu où Alexandre avait épousé Roxane et uni l’Occident à l’Orient !

Mes souvenirs m'envoient ensuite à Hérat, lors de ma première visite au mausolée d'Ansari, lieu inoubliable par sa beauté comme pour l'atmosphère qui s'en dégage. A l'entrée du sanctuaire, un chien veille, accroupi. Un chien devant un lieu saint musulman ?

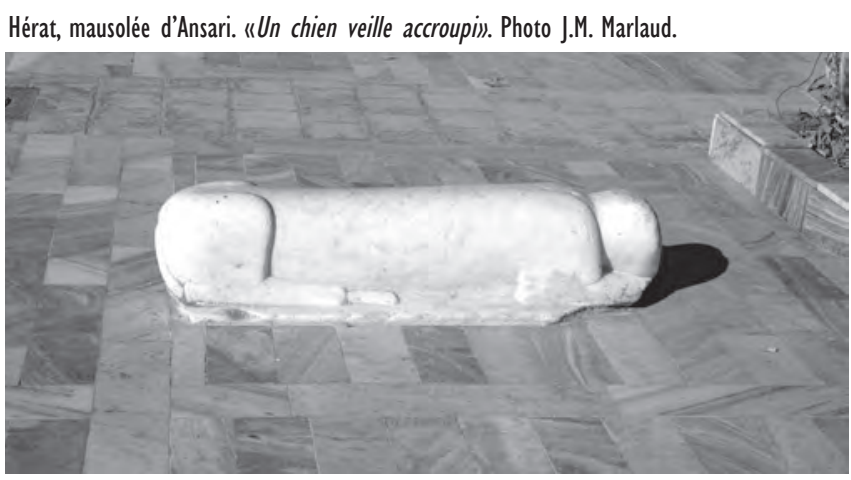




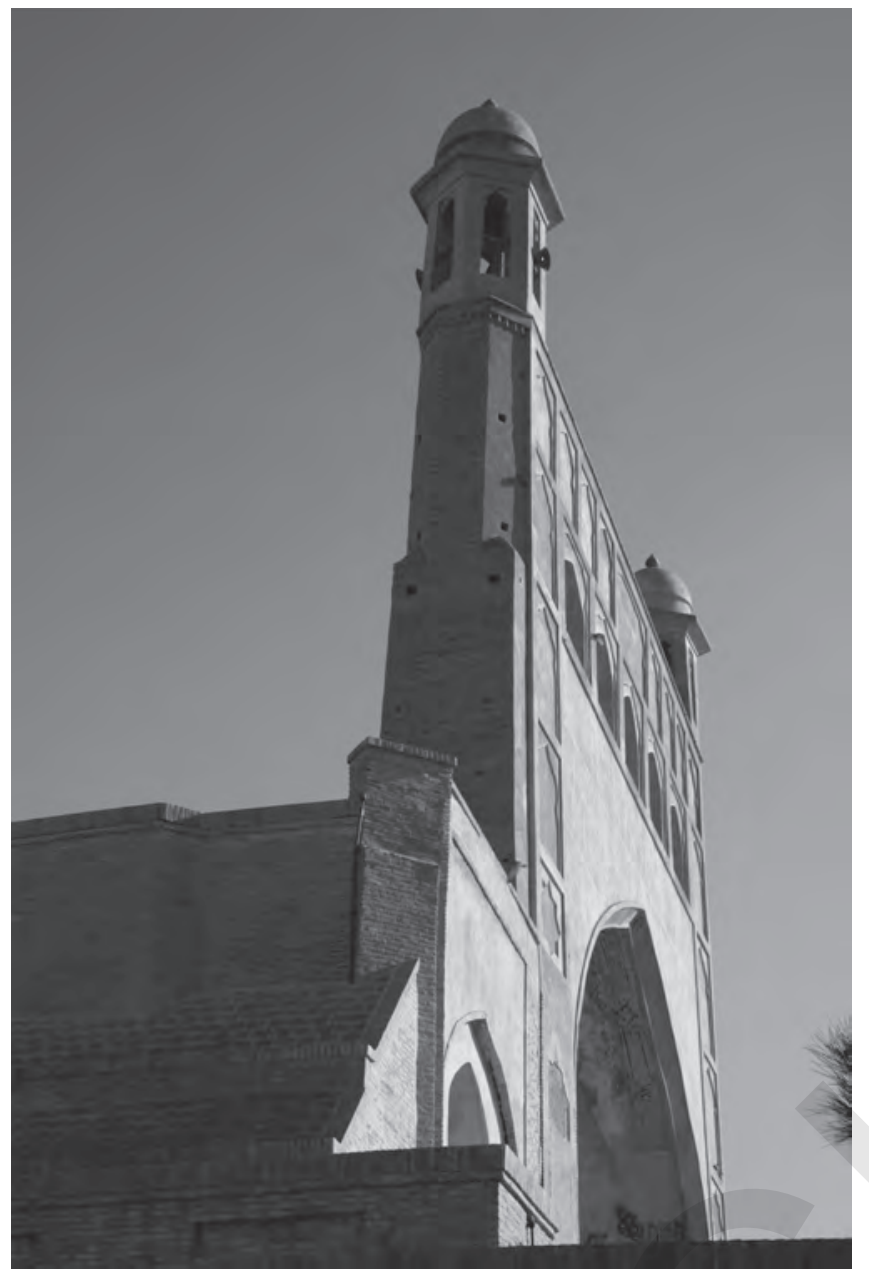

Hérat. «Ma première visite au mausolée d'Ansariı. Photo J.M. Marlaud.

Allusion, nous explique Michael Barry, à l'histoire des Sept dormants d'Ephèse, cachés dans une grotte pour se protéger de leurs persécuteurs, endormis plusieurs siècles sous la garde vigilante de leur chien, une histoire commune à l'islam et au christianisme. Un chien qui a passé toute la période des Tâlebân caché par le gardien sous une estrade de planches couvertes de plantes vertes et n'a retrouvé l'air libre que lorsqu'il ne risquait

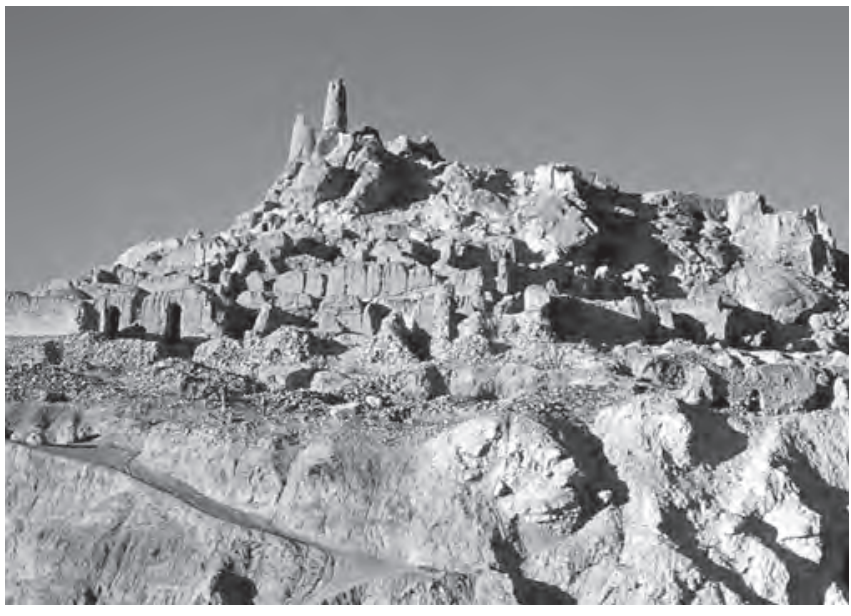

Bâmyân, la Cité des Murmures. «L'on n'entendrait plus que le murmure du vent». Photo DR.

plus d'être détruit.

Plus tard, j'ai eu la chance de visiter un autre site, Mes-e Aynak, proche de Kaboul mais difficile d'accès pour des raisons de sécurité. Un endroit que l'on n'oublie pas du fait de son immensité et de sa majesté, sous un ciel plombé, mais aussi par sa dimension symbolique car des religions différentes y ont cohabité pacifiquement : les restes d'un grand temple zoroastrien surplombent les monastères bouddhistes, au pied d'une des plus grandes réserves de cuivre du monde, déjà exploitée dans l'Antiquité. Emblématique aussi des risques que font courir l'exploitation effrénée des ressources naturelles : la ville disparut lorsque, toutes les forêts ayant été détruites, il devint impossible de produire le cuivre. Ses restes archéologiques exceptionnels partiront en poussière lorsque les Chinois, qui ont obtenu la concession, mettront la mine en exploitation.

De Bâmyân, je retiendrai la Cité des Murmures, détruite en 1221 par Gengis Khan. Les ruines, face aux grottes des Bouddhas, font revivre les récits du siège : le petit-fils de Gengis Khan frappé à mort par une flèche

Vallée du Pandjchir, tombeau de Massoud. Photo DR

Mes-e Aynak. "Ses restes archéologiques exceptionnels partiront en poussière». Photo DR

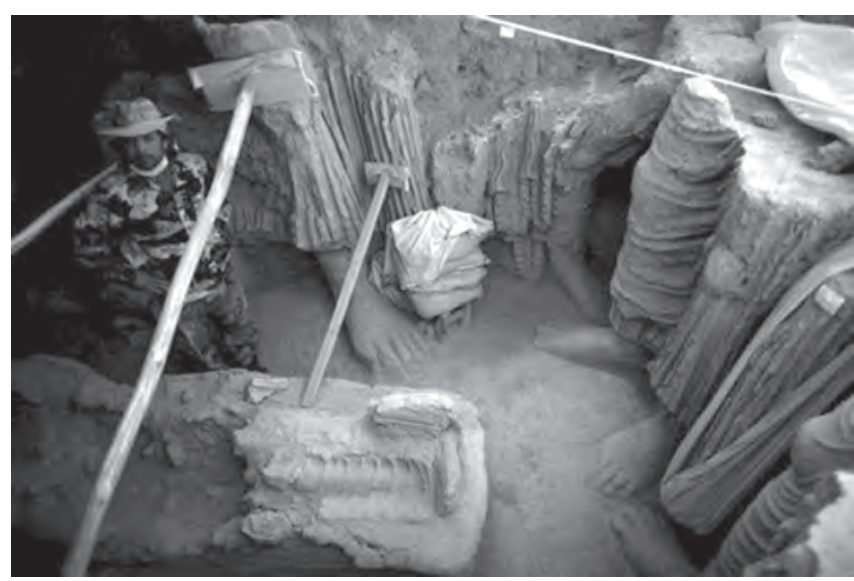

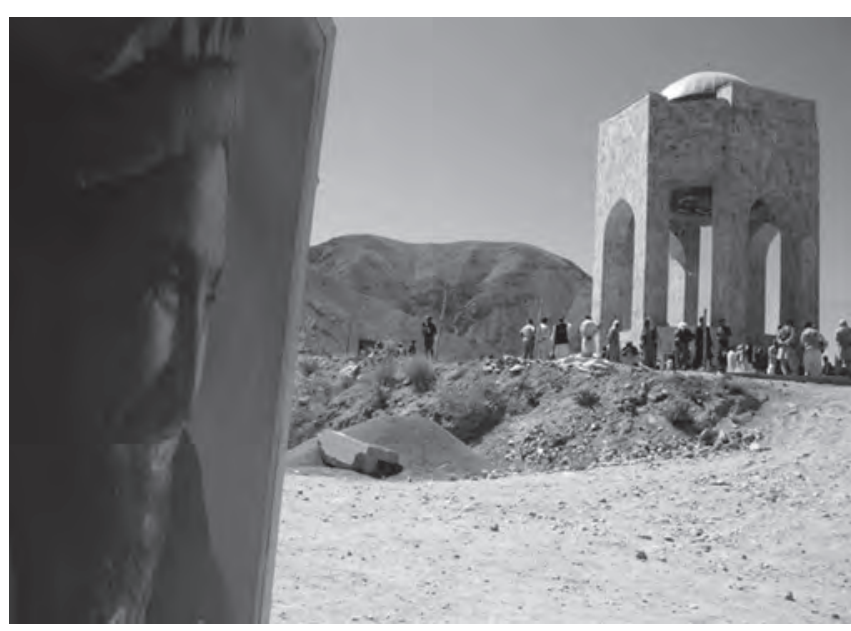

Les Nouvelles d'Afghanistan ${ }^{\circ} 157$ 


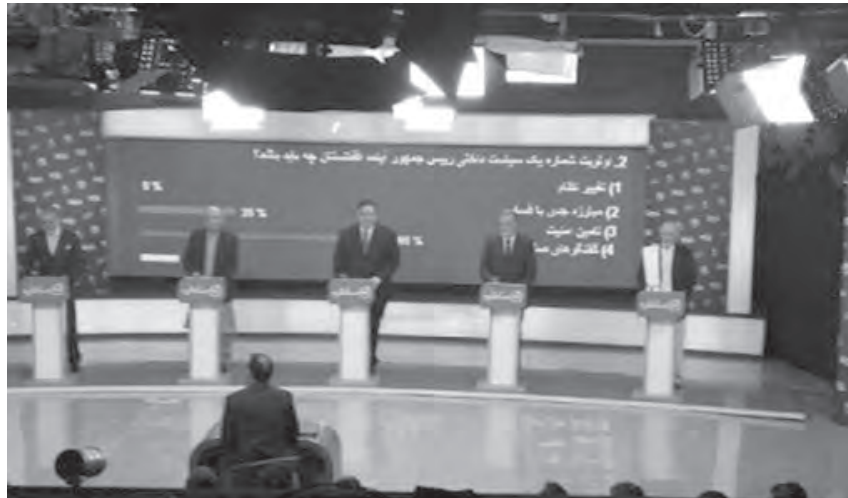

Election présidentielle, débat télévisé. «Pouvoir échanger sur leurs programmes et leur vision de l'avenir du paysı. Photo TN

qui l'atteint à l'œil, le serment de son grand-père de se venger en massacrant tous les êtres vivants, au point que l'on n'entendrait plus que le murmure du vent dans les ruines, la trahison de la fille du roi montrant aux Mongols où couper l'approvisionnement en eau de la cité... La DAFA y effectue des fouilles à la demande de l'UNESCO, démontrant ainsi sa capacité à s'intéresser au passé musulman et pas seulement bouddhiste du pays.

D’autres lieux renvoient à l'histoire plus récente : les restaurants de poissons sur les berges de l'AmouDaria, dont la tranquillité évoque l'Afghanistan des années 60, la maison de Massoud dans le Pandjchir, dominant le torrent au fond de la vallée. Car la nature est inséparable de ces évocations : comment oublier ma visite à Istalif, pour voir des écoles, à un moment qui coïncidait avec la brève floraison des arbres de Judée, nous renvoyant aux mémoires de l'Empereur moghol Babour, qui a écrit des pages si émouvantes sur ce village à cette saison?

Une dernière image : la soirée offerte à Kaboul pour mon départ par le principal think tank du pays, dans la forteresse aux neuf tours parfaitement restaurée, illuminée par des lanternes, au son d'un orchestre de musique traditionnelle.

\section{Du côté de la politique}

Mes trois années afghanes ont coïncidé avec ce qui devait être la clôture de la période post-11 septembre, avec le retrait des forces combattantes de l'OTAN et des élections présidentielles permettant le premier changement pacifique et démocratique du chef de l'Etat dans l'histoire du pays. Bien des images sont liées à ces évènements, à commencer par le Noël pour le moins inhabituel que mon épouse et moi-même avons passé le 24 décembre 2013 avec le Ministre de la Défense et tous nos militaires à KAIA, l'aéroport militaire de Kaboul, sous les immenses tentes dressées sur les pistes.

Quelque temps auparavant, j'avais effectué une visite à Bagram, principale base américaine et de l'OTAN après avoir joué le même rôle pendant l'occupation soviétique. Si elle est restée gravée dans mon esprit, c'est d'abord en raison des conditions de son déroulement : un déplacement en hélicoptère, avec gilet pare-balles, casque et bouchons d'oreilles, sous la garde vigilante d'un mitrailleur qui scrutait les collines dans l'attente d'un tir ennemi. Bien des fois je suis retourné à Bagram par la suite, non pour y visiter la base mais l'exploitation agricole qui se trouve à côté et où travaillent des compatriotes : des trajets par la route, sans ostentation, pour arriver dans une sorte de miniature persane idyllique, au milieu d'arbres en fleurs et d'abeilles rapportant dans leurs ruches tout le suc butiné. Un même lieu peut décidément se prêter à des récits bien différents !

Une dernière image est associée à Bagram, celle d’un général américain qui conclut son exposé en me remettant un dossier avec, sur la couverture, en lettres énormes : ce que vaincre signifie. Même convaincu de la défaite inéluctable des Tâlebân, cette expression m’avait semblé un peu décalée...

A la veille du premier tour des élections présidentielles, j’avais invité à la résidence de Kaboul les trois principaux candidats, afin de pouvoir échanger sur leurs programmes et leur vision de l'avenir du pays. Au-delà des propos, ces trois rencontres m’ont frappé parce qu'elles montraient les différences de personnalité entre les trois hommes qui se présentaient aux suffrages de leurs compatriotes, sans qu'il soit bien sûr question pour nous de porter des jugements de valeur sur les uns ou les autres. La diplomatie ne s'exerce pas seulement dans les bureaux mais aussi dans ces occasions plus intimes...

Les autres images liées aux élections sont plus brutales. Il y a, entre les deux tours, mon déplacement à Kandahar, où des rendez-vous avaient été pris par les Nations unies avec des personnalités locales : entretiens courtois où les arguments en faveur d'un candidat étaient présentés avec sagesse et modération, pour ne pas dire langue de bois. Mais, au milieu de nos échanges, une attaque contre les bureaux du gouverneur nous a obligés à quitter les bureaux pour nous installer dans la zone de repli, en fait un bunker sommairement aménagé où nous sommes restés de longs moments... assez en tout cas pour que, confiance et fatigue aidant, les masques tombent et les propos policés fassent place à des remarques politiquement beaucoup moins correctes !

Les tensions sont devenues très fortes au lendemain du 2ème tour, avec des accusations de fraude et des risques sérieux de dérapage. La solution trouvée pour apaiser les esprits et garantir le bon déroulement du processus fut de proposer l'observation internationale du dépouillement : 200 observateurs, dont la moitié européens, à mobiliser en quelques jours en plein mois de juillet, alors qu'aucun budget n’avait été prévu pour cela ! Grâce à l'engagement des ambassades d'Al- 
lemagne, de France et du Royaume-Uni, nous avons réussi, et l’Union Européenne, si souvent critiquée, a démontré sa capacité de mobilisation. Mais il fallait quinze jours avant l'arrivée de ces observateurs et il était impossible d'attendre. Les ambassades ont donc décidé de mobiliser leurs propres agents pour commencer le dépouillement sans délai. C’est ainsi que je me suis trouvé à Daroulamân, au siège de la commission nationale électorale, rendant visite à mes collègues de l'ambassade volontaires pour l'exercice. Des collègues engagés et courageux : dans l'improvisation, rien n'avait été prévu pour leur confort, même pas de l'eau, alors que le soleil de juillet sur les toits de tôle rendait la chaleur insupportable. Et comment oublier la menace, alors que tous ces bulletins réunis en un seul lieu constituaient le meilleur démenti apporté par le peuple afghan aux prétentions des Tâlebân ?

La dernière image liée à la politique est aussi la dernière de mon séjour en Afghanistan : la réunion à Kaboul, au siège des Nations unies, entre les négociateurs du gouvernement et de Hekmatyâr, pour signer le document ouvrant la voie à un processus de réconciliation. Cette rencontre, le soir précédant mon départ définitif, marquait une étape importante d'un processus commencé plusieurs mois auparavant à l'ambassade de France. Une réconciliation controversée mais qui montrait un chemin aux Tâlebân qui seraient convaincus que la victoire militaire est impossible.

\section{La plainte du raseau}

Ecoute ce que raconte la flîte de roseau Des séparations elle se plaint

«Depuis que L'on m'a coupé de la roselière Hommes et femmes ont gémi par mon intermédiaire.

Je cherche une poitrine déchirée pour que je hi explique la douleur du désir.

Qui demeure loin de son origine cherche le jour oì il la retromnera

Je me suis plainte dans chaque assemblée, j'ai été unie aux gens heureux et aux malheurenx,

Chacun est devenu mon ami. mais personne n'a recherché mes secrets.

Mon secret n'est pas loin de ma plainte mais les yeux et les oreilles ne pervent le percevoir.

C'est du feu, le son de la flute, ce n'est pas du vent,

Qui n'a ce fen qu'il soit réduit à néant

C'est le feu de l'amour qui se troune dans la flute

C'est le bovillonnement de l'amour qui se troune dans le vin. 》

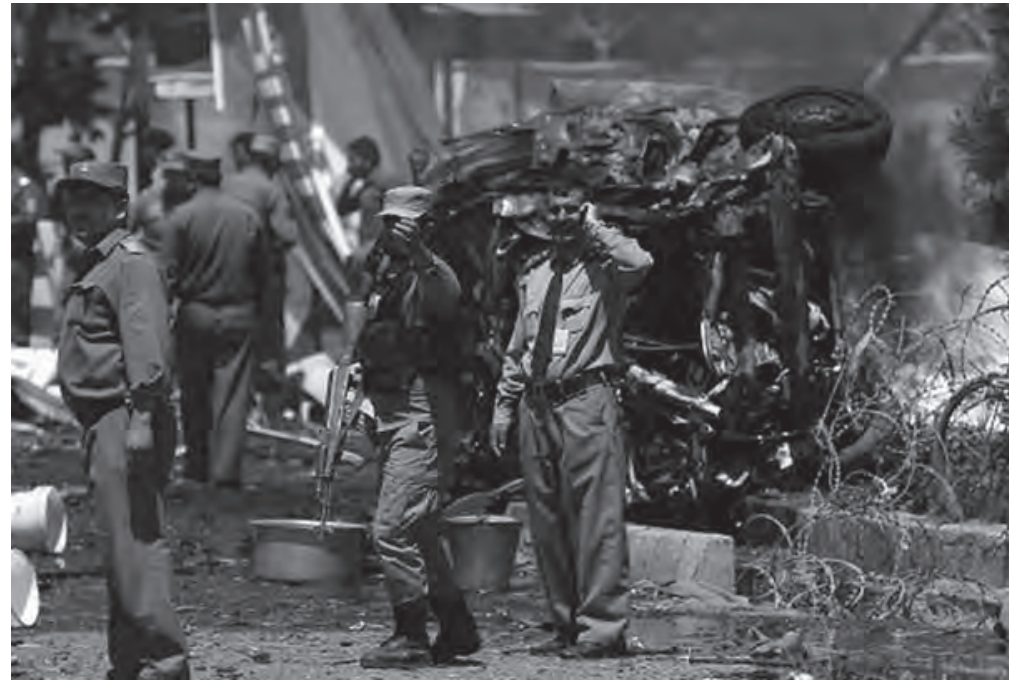

Attentat à Kaboul. «D'autres images sont liées à la violence». Photo DR

\section{La violence}

D’autres images, à Kaboul, sont liées à la violence : l'attentat à l'Institut français, fauteuils défoncés et parois éventrées avec, dans l'allée, le corps sans vie d'une victime tuée sur le coup. La mort, lors de l'attentat contre la Taverne du Liban, du représentant du FMI, lui-même Libanais, grand ami de notre pays - où il est d'ailleurs inhumé - mais aussi de l'Afghanistan où, année après année, il avait demandé à prolonger son séjour. La solidarité démontrée par les Afghans à notre égard après les attentats du 13 novembre, qui s'est manifestée par l'initiative d'organiser à la résidence un concert de musique soufie en mémoire des victimes. Quelle émotion en entendant le chanteur prononcer les vers si poignants de Roumi qui disent la douleur de la séparation que symbolise la flûte de roseau !

Comment oublier la visite d'un camp de déplacés cernés par les hautes tours d'un projet immobilier, à qui toute aide était refusée parce que le promoteur entendait récupérer son terrain, alors que l’hiver arrivait ? Ou encore, à la sortie du havre de beauté et de tradition que sont les maisons restaurées et les ateliers d'art de Turquoise Mountain, le monument à la mémoire de Farkhunda, jeune femme lynchée par une foule constituée de jeunes urbains, capables de tout filmer sur leurs smartphones et dont on se demande s'ils ne constituent pas une nouvelle espèce de monstres ?

\section{Projets}

Je l'ai dit, l'ordre de présentation n'est pas neutre. C'est donc délibérément que j'achèverai avec le souvenir de visites à des projets qui sont l'espoir de ce pays.

Mon souvenir le plus fort me ramène en Kapisa, lors de la cérémonie de remise de leurs diplômes à de jeunes infirmières. C'était mon premier déplacement dans 


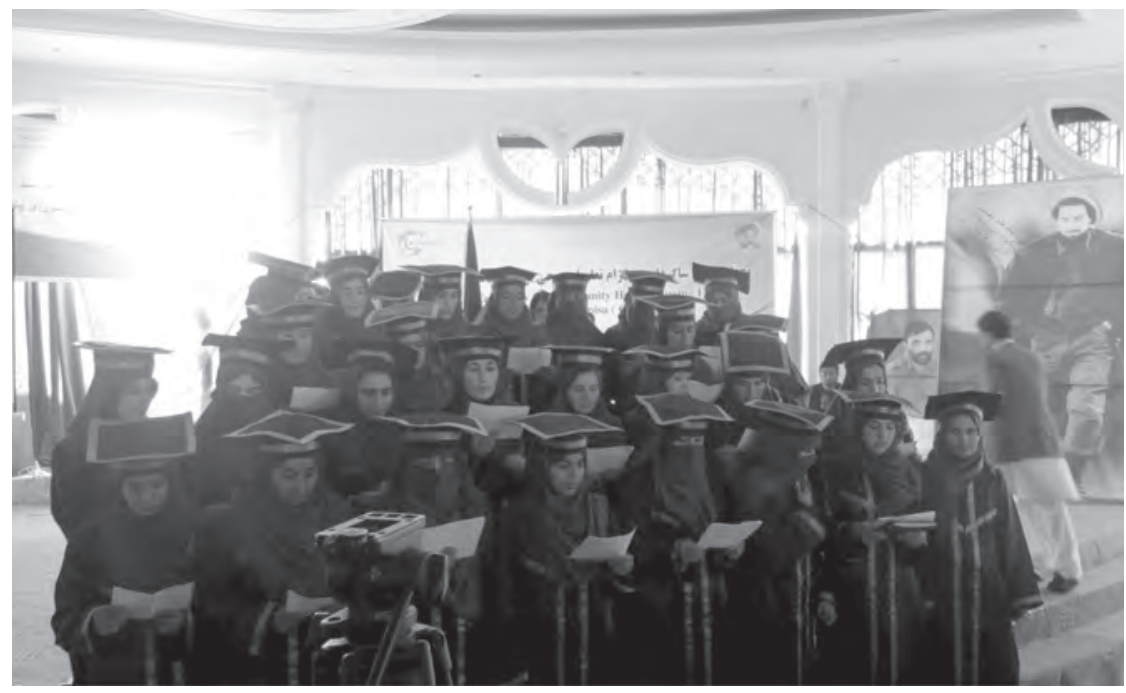

Remise de diplomes.

«Des petites toques copiées

sur les universités américaines».

Photo DR

sance d'expression propre à un peuple où l'art oratoire est développé au plus haut point?

A Kaboul, bien sûr, beaucoup de souvenirs ont trait à l'hôpital de la mère et de l'enfant, fleuron de notre

cette région où s'est longtemps concentrée l’aide française mais qui, aujourd'hui, est difficile d'accès. Après avoir visité le nouveau bâtiment de la direction de la Santé, construit avec notre aide, nous sommes allés dans les locaux du gouverneur où attendait un groupe d'une vingtaine de jeunes filles, vêtues de tenues particulièrement peu seyantes, d'une couleur étrange avec, perchées sur leur voile, des petites toques copiées sur les universités américaines. Je n’ai pu m’empêcher de ricaner, sentiment dont j'ai eu honte lorsqu'on m'a expliqué que la lauréate de la promotion venait, comme trois de ses compagnes, de la vallée de Tagâb. Une zone bien connue de nos militaires pour la pression que lui font subir les Tâlebân et où le conservatisme le plus étroit est la règle. Il avait fallu retarder de plusieurs mois le début de la formation, le temps de convaincre les notables de laisser les quatre candidates venir suivre leurs études en internat. Pour la première fois dans l'histoire, les femmes de la vallée auraient accès à des infirmières et des sages-femmes !

Comment ne pas évoquer les rencontres à Tcharikar avec les directrices d'écoles soutenues par Afrane, de fortes personnalités, très différentes les unes des autres, mais ayant en commun la clarté de leur vision et une ai- coopération, fruit d'une collaboration réussie entre deux Etats (l'Afghanistan et la France), l'Aga Khan et l'ONG la Chaîne de l'Espoir. Dans la Maison de l'enfant, où des jeunes attendent d'être opérés ou se remettent de l'intervention chirurgicale, c'est une Babel où se mêlent toutes les régions, toutes les langues du pays, dans une apparente bonne humeur permanente malgré les épreuves.

Tous les projets ne portent pas sur la santé ou l'éducation. A Djalalabad, j’ai été l’invité de la prodigieuse hospitalité afghane chez un entrepreneur qui a créé, avec un ami français, une entreprise d'huiles essentielles de fleurs d'oranger et d'essence de roses, exportées vers la France et le Canada. Il fait travailler en saison des centaines de familles paysannes et les immenses alambics présents dans son jardin témoignent de cette activité qui complète leurs revenus. A Yakaolang, dans la province de Bâmyân, trois ONG françaises contribuent au développement de ces zones de montagnes froides et rudes, sur financement de l'AFD. J'y ai été le discret témoin d'une séance de vulgarisation des méthodes d'élevage, dans une petite mosquée rurale, seul lieu de rencontre de la communauté où les femmes semblaient heureuses de se retrouver autant que d'apprendre.
Kaboul, Hôpital de la Mère et de l'Enfant. «Fleuron de notre coopération». Photo Chaïne de l'Espoir
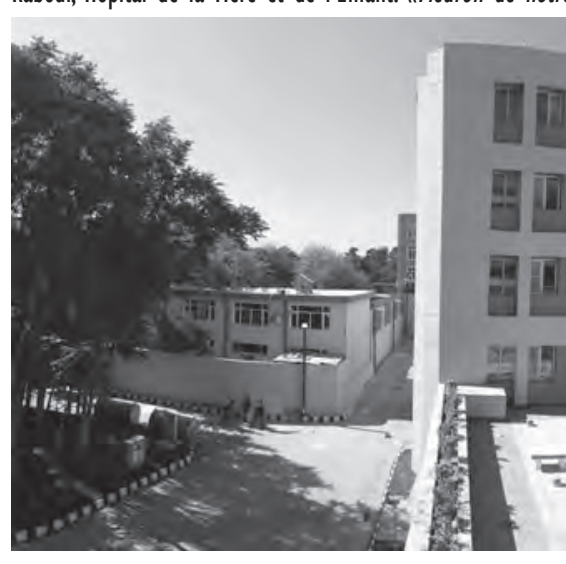

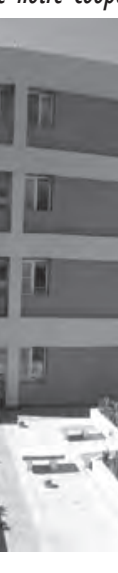

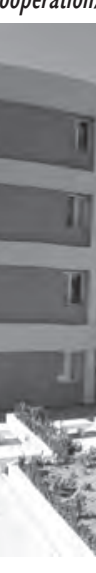

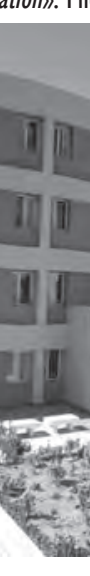

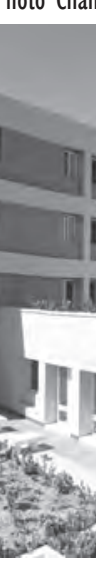

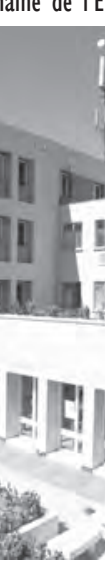

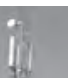

Pour achever ce parcours, je reviendrai à cette image de l’Afghanistan millénaire que nous portons au fond de nous et qui est aussi une réalité contemporaine, avec la vision de ces artisans qui, dans la mosquée bleue de Herat, sans relâche, réparent et refont carreaux de céramique ou sculptures et semblent tout droit sortis des miniatures de Behzad. 


\section{Mohamad Moussa Chafiq}

par Zia FARHANG*

Un colloque s'est tenu au début de l'année en Afghanistan pour rendre hommage à Moussa Chafiq qui participa à la rédaction de la Constitution de 1964, fut plus tard Premier ministre, puis fut éliminé sans procès par le régime communiste. Zia Farhang, qui le connut quand il était adolescent, retrace ici son itinéraire.

Parmi les figures politiques marquantes de la seconde moitié du 20e siècle de l'Afghanistan, Mohamad Moussa Chafiq occupe sans doute une des premières places.

Mohamad Moussa Chafiq naquit le 28 mai 1929 à Kaboul dans une famille religieuse et passa son enfance et son adolescence à Badri, son village d'origine, dans la province de Nangarhâr. Son père, Maolawi Mohamad Ibrahim, un théologien très respecté, exerçait la fonction de juge et jouissait d'une grande réputation d'intégrité et d'équité.

Le jeune Chafiq, esprit brillant, suivit tout naturellement le chemin de son père et dès la fin de ses études secondaires, qu'il accomplit dans une école religieuse, partit pour l'Egypte pour y étudier le droit musulman à l'université Al-Azhar. Après avoir terminé ses études en Egypte, il les poursuivit aux Etats-Unis et y étudia le droit international à l'université de Columbia.

\section{Le désir de réformes profondes}

A son retour en Afghanistan, Moussa Chafiq entama une carrière classique de jeune cadre afghan éduqué et fut nommé directeur de la section chargée de l'élaboration des lois au sein du ministère de la Justice. Par ailleurs, l'université de Kaboul le sollicita pour y enseigner le droit international. Ainsi, en apparence, la carrière professionnelle de ce jeune cadre semblait être

* Ingénieur civil.

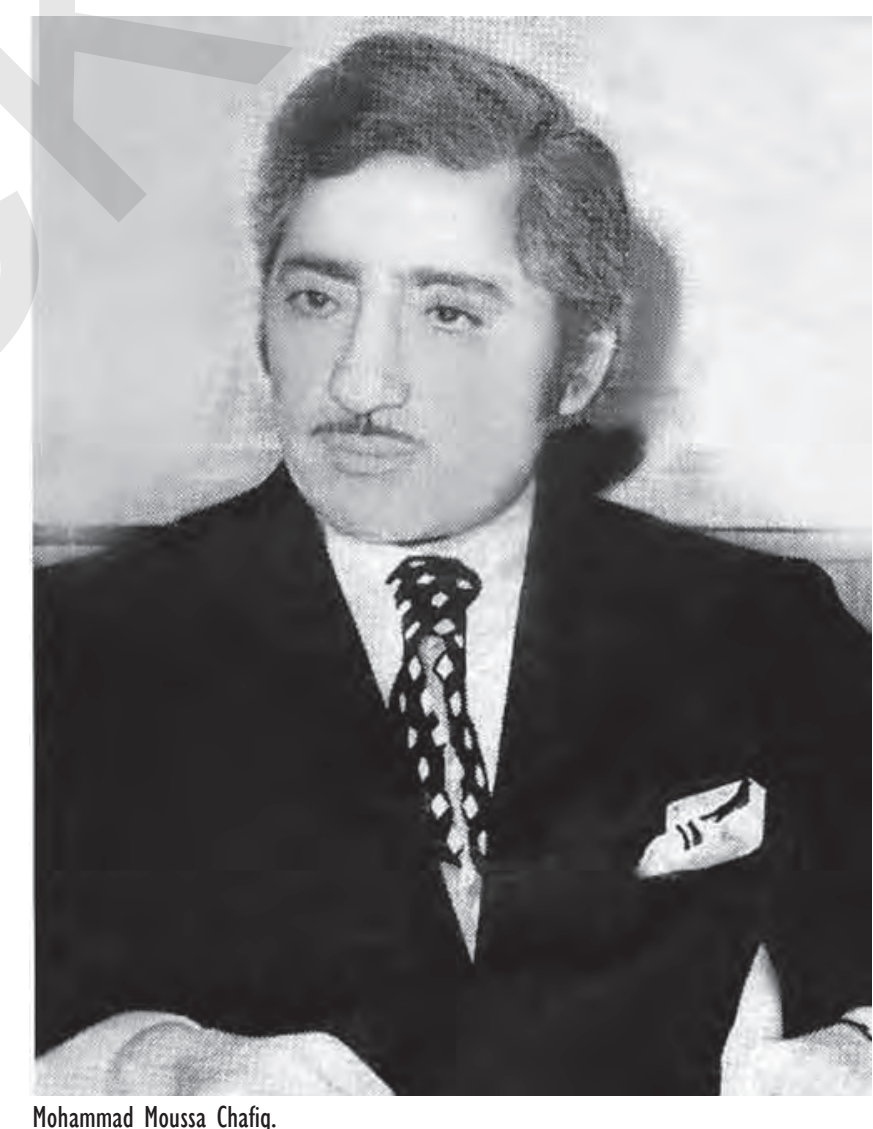

toute tracée et allait lui permettre de gravir les échelons administratifs un à un pour accéder peut être un jour à un poste ministériel. Il convient de noter ici que nous parlons d'une époque où le plus haut poste qu'une per- 


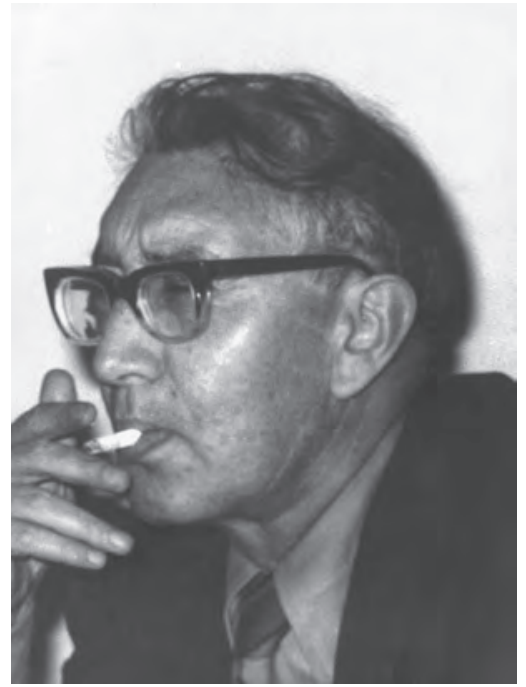

Sediq Farhang. «Chafiq dui demanda ce qu'il pensait d'un changement de régime en un régime démocratiquel). sonne non-membre de la famille régnante de l'Afghanistan pouvait occuper était précisément un poste ministériel ${ }^{1}$. Mais les apparences étaient trompeuses car le jeune Chafiq n'aspirait pas seulement à faire une brillante carrière administrative mais souhaitait aussi œuvrer pour introduire de profondes réformes dans le pays et essayer de démocratiser le poussiéreux régime royal, héritage du 19e siècle.

On sait qu’à cette même époque le monarque afghan, le roi Zâher Châh, évoquait et peut-être souhaitait un tournant démocratique à son régime mais on ignore s'il en avait parlé avec Chafiq. Connaissait-il Chafiq ? Vraisemblablement oui car Chafiq était très proche de l'influente famille Guélâni laquelle était très proche du roi. Toujours est-t-il que, bien que ce fût très dangereux - le premier ministre de l'époque, le prince Daoud, ne tolérait aucune activité politique dans le pays - Chafiq entreprit de consulter quelques intellectuels afghans afin d'étudier la possibilité de réformer le régime. Cela se passait à la fin des années 50, période où Moussa Chafiq, pour améliorer son ordinaire, travaillait de $17 \mathrm{~h}$ à 20h à la radio afghane pour y préparer le programme en langue arabe. A cette même époque M. Sediq Farhang travaillait aussi à la radio afghane pour la même raison et y préparait le texte persan des informations de 20h. Farhang, qui a fait la connaissance de Chafiq à cette occasion, se souvient dans ses mémoires ${ }^{2}$ qu'un soir, alors qu'ensemble ils quittaient à pied les bureaux de la radio, Chafiq lui demanda à brûle-pourpoint ce qu'il pensait d'un changement du régime en un régime démocratique du type de ce que les intellectuels - dont Farhang lui-même - avaient réclamé dix ans plus tôt au sein du parti Watan. Intéressé et très surpris, Farhang dit lui avoir répondu que, compte tenu de la situation politique et sociale du pays, l'éventualité d'une telle réforme lui paressait très faible. Ce à quoi Chafiq lui avait rétorqué qu’il serait intéressant qu’ils étudient ensemble cette éventualité aussi faible fût-elle. Ce témoignage démontre qu'encouragé ou non par le roi, Chafiq s'était résolu à travailler à la démocratisation de son pays et on peut dire qu'il y consacra le reste de sa vie.

\section{Une action pour la démocratie}

Moussa Chafiq fut un acteur éminent de la décennie constitutionnelle. Membre très actif du comité rédactionnel de la constitution de 1964, il défendit jusqu'au bout les acquis de ladite constitution. En automne 1972, alors qu'il était sur le point d'être nommé Premier ministre, il n’hésita pas à organiser une réunion secrète de travail à Paris en présence de MM. Fougère ${ }^{3}$ et Farhang où ils passèrent en revue les difficultés de la mise en application correcte de la constitution de 1964. A cette occasion il prit, entre autre, l'engagement d'obtenir du roi la promulgation des lois déjà approuvées par le Parlement - notamment la loi sur les partis politiques qui restaient bloquées dans le cabinet royal et d'édicter une nouvelle loi délimitant de manière plus précise les compétences des trois organes de l'Etat, afin de juguler les agissements anarchiques des membres de la Wolossi Djerga (Assemblée Nationale).

Mohamad Moussa Chafiq avait un profond sens

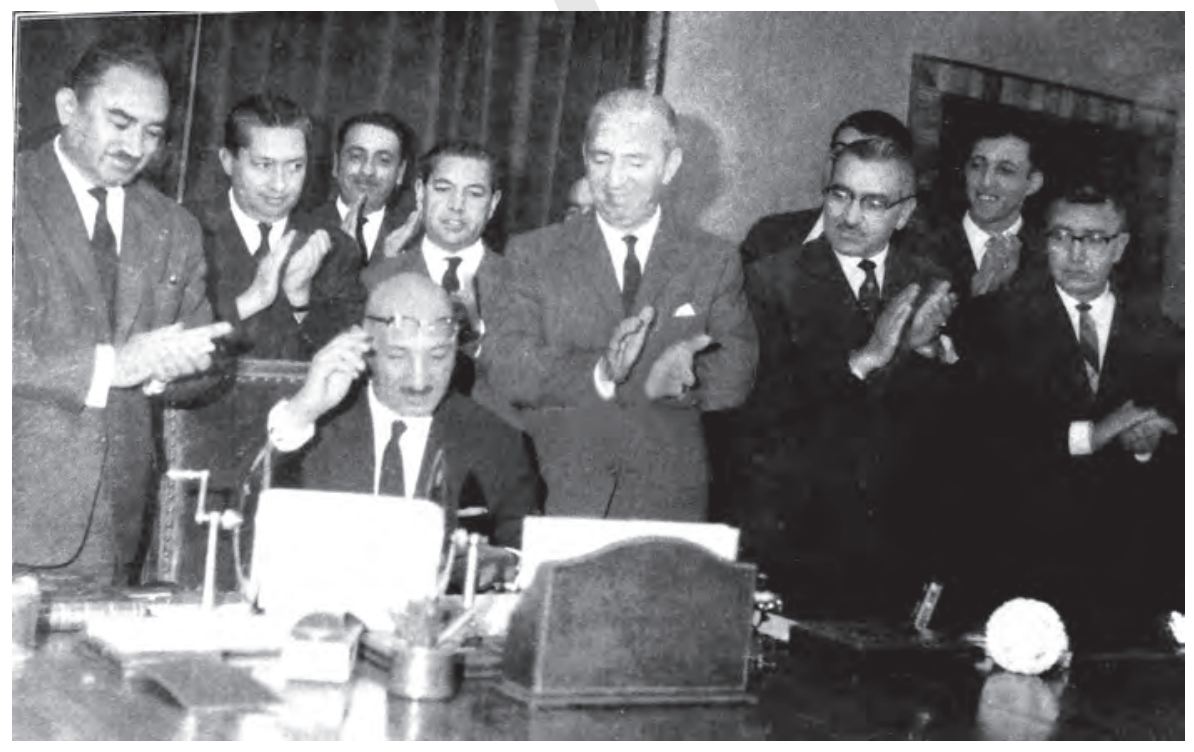


de l'Etat et une foi immense en la capacité du peuple afghan de trouver la place qui lui revenait au sein du concert des Nations. A l'occasion du discours inaugural de son mandat de Premier ministre il déclara croire fermement à la « sagesse innée du peuple afghan » et il y croyait vraiment.

Chafiq attachait également une grande importance au prestige dû au rang des responsables de l'Etat. Ainsi, selon M. Sayed Mohamad Maywand Ouloumi qui fut un de ses proches collaborateurs, un jour, alors que le Premier ministre était en réunion de travail, son directeur de cabinet l'informa que Sa Majesté la Reine était au téléphone et désirait s'entretenir avec lui. Chafiq, sans la moindre hésitation, lui dit d’informer Sa Majesté qu’il n’était pas en mesure de lui parler immédiatement et qu'il l'appellerait dès que possible. Il n'était pas aux ordres.

Durant la courte période où il occupa le poste de Premier ministre (un peu plus de sept mois en 1972-73) Moussa Chafiq fit la démonstration de sa très grande capacité à diriger le pays. Parmi ses plus grandes réalisations on peut, sans conteste, citer l'accord sur le partage des eaux du fleuve Helmand avec l'Iran. Les négociations concernant le partage des eaux du fleuve Helmand dataient du 19e siècle et avaient échoué à trois reprises. Lors de ces négociations, la meilleure proposition qu'on avait faite aux Afghans avait été formulée en 1872 par l'arbitre anglais Henry McMahon qui accordait $66.5 \%$ des eaux du fleuve à l'Afghanistan. Cette proposition avait été refusée par la partie iranienne. Le gouvernement Chafiq réussit à obtenir que $77.5 \%$ des eaux du fleuve soit attribuées à l'Afghanistan. De surcroît il obtint que l'Iran accorde une compensation financière à l'Afghanistan pour les eaux excédentaires que l'Afghanistan ne pouvait utiliser et qui tout naturellement se déversaient en Iran, ce qui contribuait à la création de richesses dans ce pays. Bien que passablement chahuté par les opposants à la constitution de 1964 - les partisans du Prince Daoud qui avait été définitivement écarté du pouvoir par l'article 24 de ladite constitution - et par les communistes qui étaient hostiles à l'éloignement d'avec l'URSS et le taxaient de trahison, le gouvernement Chafiq parvint à faire ratifier l'accord en question par le Parlement afghan.

\section{Un tournant diplomatique qui lui coûtera cher}

Un autre chantier très difficile et délicat auquel s'attaqua M. Chafiq fut le changement de l'orientation de la politique étrangère de l'Afghanistan. Ainsi, le gouvernement Chafiq, sans prendre ostensiblement ses distances avec l'URSS, commença à se rapprocher de l'Ouest et en particulier des pays arabo-musulmans.
Concernant le Pakistan, Chafiq fit montre d'un grand courage politique et déclara dans un entretien avec le journal «Afghan Mellat », organe du parti nationaliste pachtoune du même nom, que « Nous ne pouvons pas sacrifier l'Afghanistan sur l'autel du Pachtounistan!"». Il réussit également, comme indiqué plus haut, à éliminer le seul point d'achoppement qui ternissait les relations afghano-iraniennes.

On a parfois taxé Chafiq d'avoir eu de la sympathie pour les Frères Musulmans. Pour preuve ses détracteurs avancent le fait qu'il introduisit la diffusion de l'appel à la prière à la radio afghane et le fait qu'un certain nombre de jeunes activistes fondamentalistes musulmans dont M. Golbuddin Hekmatyâr, jetés en prison sans avoir été jugés, furent libérés sur son ordre. A bien $\mathrm{y}$ réfléchir force est de constater qu'à cette époque il y avait un grand déséquilibre entre l'Afghanistan des villes et l'Afghanistan profond, celui des campagnes. En ville la jeunesse se donnait du « camarade » et s'enorgueillissait d'être progressiste, voire communiste et sympathisant ou encore membre d'un des deux partis Partcham ou Khalq ouvertement marxistes-léninistes, alors que la campagne, c'est-à-dire l'immense majorité du peuple afghan, demeurait très pieuse et profondément traditionaliste. Dès lors il est permis de penser que les gestes du gouvernement Chafiq en faveur de la religion et des religieux avaient essentiellement pour but un nécessaire rééquilibrage social et politique et non l'introduction du fondamentalisme dans le pays.

Mohamad Moussa Chafiq était un musulman modéré et un intellectuel épris de littérature, notamment de littérature persane. Polyglotte (il parlait outre le pachto et le dari, l'arabe, l'anglais et le français) ${ }^{4}$, il aimait écrire. On connait de lui un livre intitulé « Abqaryan - Les gens cultivés » publié à Kaboul. Il nous laisse également des poèmes écrits dans les deux langues dari et pachto qui n’ont pas encore été publiés.

Mohamad Moussa Chafiq fut assassiné par le régime sanguinaire de Taraki. On ne connait pas la date exacte de son assassinat mais Il est de notoriété publique qu'au moment de sa présentation devant le peloton d'exécution il demanda à connaitre la raison pour laquelle on le tuait. Il lui fut répondu : « Pour avoir signé le traité du partage des eaux de Helmand !».

Il avait à peine 50 ans.

1- Le poste de Premier ministre était réservé jusqu'en 1964 aux membres de la famille royale.

2- Khâterât, M.S. Farhang, en dari, 2015, Edition Teesa - Téhéran - 623 pages. Disponible à AFRANE.

3- Membre du Conseil d'Etat qui fut associé à la rédaction de la Constitution afghane de 1964.

4- Il a séjourné à Paris durant six mois pour y apprendre le français. Cela se passait avant qu'il soit nommé ministre des Affaires étrangères durant une petite traversée du désert. Ce devait être en 1967/68. 


\title{
L'intervention militaire soviétique vue de Moscou
}

\author{
par Gilles ROSSIGNOL*
}

\section{L'intervention en Afghanistan ne faisait pas l'unanimité au sein du Politburo et de l'état-major de l'armée soviétique. L'état de déliquescence du parti commu- niste afghan et la nécessité de conserver l'Afghanistan dans le giron de l'URSS en furent la justification.}

L'article ci-après ne constitue pas une histoire de l'intervention soviétique en Afghanistan. À partir de neuf documents parmi ceux déclassifiés en 1992 sur ordre de Boris Eltsine, traduits par la National Security Agency ${ }^{1}$, il s'agit seulement d'analyser les principales étapes de l'évolution des positions du Politburo sur la question.

\section{L'affaire d'Hérat}

Le 9 mars 1979, un envoyé de Sebghatollah Modjaddedi, autorité religieuse très respectée, dont la famille avait été massacrée par les communistes après la " révolution de Saor », arrive à Hérat. Venu de Peshawar, il est porteur d'une missive invitant la population à un soulèvement général. La révolte éclate le 14 mars et s'étend rapidement. Des membres du parti communiste, des fonctionnaires du régime proches du parti, ainsi que des conseillers soviétiques et leurs familles sont exécutés. Les insurgés subissent de lourdes pertes, mais parviennent à rallier à leur cause des militaires. La révolte sera matée, noyée dans le sang, le 20 mars²$^{2}$.

Le 17 mars 1979, le Politburo, l'organe décisionnel du pouvoir soviétique, se réunit avec pour ordre du jour : « Détérioration de la situation en Afghanistan et les possibles réponses de notre part ». Son président, Léonid Brejnev, s'excuse de l'avoir convoqué à une heure inhabituelle et de ne pouvoir y assister ${ }^{3}$, mais il

* Ancien président d'AFRANE. sera présent le lendemain, déclare Kirilenko, ombre de Brejnev au sein du parti communiste de l'URSS, aux quatorze autres membres. Il leur dit que la situation est urgente, qu'ils ont devant eux un certain nombre de propositions d'action rédigées par Gromyko, ministre des Affaires étrangères, Andropov, chef du KGB, et Ustinov, ministre de la Défense.

Ustinov décrit une situation très critique à Hérat où la 17e division de l'armée afghane s'est désintégrée. Selon les informations de leur conseiller militaire en chef à Kaboul, Gorelov, et de leur chargé d'affaires, Alekseiev, un régiment d'artillerie et un autre d'infanterie se sont ralliés aux insurgés. Gromyko ajoute que « des bandes de terroristes et de saboteurs infiltrés depuis le Pakistan, entraînés et armés non seulement par le Pakistan, mais aussi par la Chine, les États-Unis et l'Iran, commettent des atrocités dans Hérat ». Ils ont joint leurs forces, précise-t-il, à celles de la contre-révolution provinciale, composée pour la plupart de « religieux fanatiques ». Ces insurgés, conclut-il sur un ton dramatique, sont « des milliers, littéralement des milliers ».

À onze heures du matin, explique Gromyko, il a eu une conversation avec Hafizoullah Amin, « l'adjoint de Taraki, ministre des Affaires étrangères ", qui l'a assuré, " avec un calme olympien » que la situation n'était pas si grave, que l'armée la contrôlait, « et ainsi de suite »... « En un mot, observe de façon acide Kirilenko, le gouvernement afghan ne manifeste pas la moindre inquiétude à propos de la situation ». 
« Exactement », approuve Gromyko, Amin lui ayant assuré que « pas un seul incident n'avait été rapporté par les gouverneurs provinciaux, tous fidèles au gouvernement ». Agacé par l'attitude rassurante d'Amin, Gromyko ajoute qu'il a obtenu deux ou trois heures plus tard confirmation que « le chaos régnait à Hérat » et que Taraki, qui se trouve alors à la conférence des pays non alignés à La Havane, avait demandé aux chefs de mission soviétiques à Kaboul des armes, des munitions, des rations, tout en indiquant que des soutiens aériens et au sol seraient les bienvenus. « En d'autres termes, conclut Gromyko, ce que l'on nous demande, c'est l'engagement de nos forces aériennes et terrestres ».

Évoquant ensuite le problème de l'aide à l'Afghanistan, il déclare sans ambiguïté qu' " en aucun cas, nous ne devons perdre l'Afghanistan ", car cela constituerait un sérieux revers pour la politique étrangère de l'URSS. Il ajoute enfin que l'état de déliquescence du gouvernement afghan est préoccupant. Les nombreux « conseillers soviétiques » présents à Kaboul le confirment.

Une discussion s'engage. Ustinov s'inquiète de la situation à Hérat, mais aussi dans le Paktia. Taraki a confirmé que la situation insurrectionnelle s'étendait et qu'il attendait une " réponse majeure de la part de l'URSS sous forme d'une intervention, terrestre et aérienne ». L'échange se poursuit. La première proposition concrète viendra de Kossyguine : il insiste pour que la livraison d'armes prévue pour avril soit immédiate et demande que le prix d'achat du gaz naturel à l'Afghanistan soit augmenté, en sorte que le gouvernement afghan puisse payer le blé. Il ajoute que l'URSS a promis d'en fournir 75000 tonnes. Il propose de porter ce chiffre à 100000 tonnes.

Kossyguine fait alors part de ses réserves sur l'attitude de Taraki et d'Amin. Il faut, dit-il, que l'on parle avec eux des fautes qui ont conduit à cette situation. " Ils ont continué à exécuter ceux qui n’étaient pas d'accord avec eux ; ils ont tué pratiquement tous les chefs, pas seulement les chefs, mais aussi les cadres moyens du parti Partcham ».

Tous les participants approuvent et conviennent qu'il ne faut pas intervenir militairement, mais se préparer à toute éventualité en déployant deux divisions sur la frontière afghane. Kirilenko ajoute qu'il faudra inviter Taraki à changer de tactique politique et d'arrêter "les exécutions les tortures sur une grande échelle ». L'échange se poursuit. Les participants demandent finalement à Kossyguine de s’entretenir avec Taraki.

Le lendemain, Kossyguine rend compte de deux entretiens téléphoniques avec Taraki qui se trouve tou-

jours à La Havane. Kossyguine s'est enquis de savoir s'il aurait les équipages compétents pour faire manœuvrer les chars et véhicules blindés demandés. Taraki a répondu par la négative et a suggéré que l'URSS envoie des équipages de soldats tadjiks revêtus d'uniformes afghans. Kossyguine, qui a repoussé cette éventualité, a interrogé son interlocuteur sur la possibilité de former à Kaboul, dont la population est alors de 1,2 million d'habitants, une division avec des hommes en âge de servir. Impossible, lui a répondu Taraki, car les Kaboulis sont surtout des commerçants et des artisans, pas des ouvriers...

Ustinov observe au passage que le gouvernement afghan n'a pas pris la mesure du poids des musulmans fondamentalistes. Ce que confirme avec autorité Andropov : " Il est parfaitement clair pour nous tous que l'Afghanistan n'est pas en état de résoudre tous les problèmes auxquels il doit faire face par le socialisme. L’économie est arriérée, la religion musulmane domine, et pratiquement toute la population rurale est illettrée ». Il conclut que dans tous les cas, l’URSS ne peut pas prendre le risque d'une intervention militaire. Gromyko s'associe à cette déclaration et précise en outre que l'Afghanistan n'a subi aucune agression, que c'est un conflit révolutionnaire interne, ce qui exclut la possibilité de mettre en oeuvre les accords de sécurité collective.

\section{Taraki à Moscou}

Kossyguine propose alors que l'on invite Taraki à faire escale à Moscou lors de son retour de La Havane, pour lui dire qu'on va l'aider par tous les moyens, mais que l'on n'enverra pas de troupes. Kirilenko surenchérit : « Le gouvernement afghan n’a rien fait pour normaliser la situation. Et il a une armée de 100000 hommes ! Qu'a-t-il fait ? Qu'a-t-il fait de bon ? Pratiquement rien. Et après tout, camarades, nous donnons une très très grosse aide à l'Afghanistan ». Le comité 


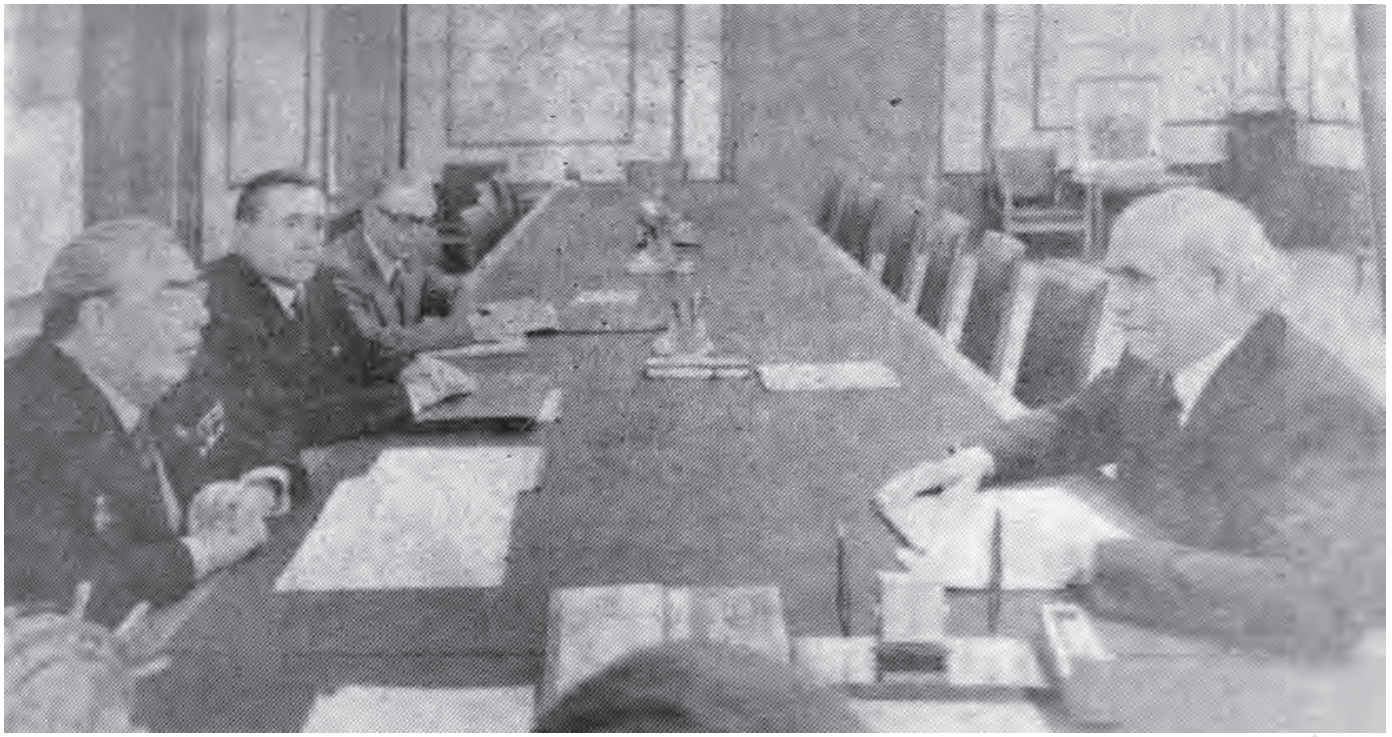

Taraki face à Léonid Brejnev et Kossyguine à Moscou. (extrait du Kabul Times).

décide in fine d'inviter Taraki.

Le lendemain, Brejnev explique qu'il a signé les documents nécessaires pour l'aide supplémentaire à l'Afghanistan et confirme la décision du Politburo de ne pas intervenir militairement. Gromyko ajoute qu'il paraît évident que les incidents de Hérat sont dus à l'action des gouvernements américain, chinois, pakistanais et iranien. Mais une intervention militaire placerait l'URSS en position d'agresseur, dit-il. Elle mettrait en péril les négociations SALT II, les rencontres prévues avec les présidents Carter et Giscard d'Estaing, ainsi que les relations avec les autres pays occidentaux.

Taraki arrive à Moscou le 20 mars. Une réunion est aussitôt organisée avec Kossyguine, Gromyko, Ustinov et Ponomarev. Kossyguine mène le débat. Il commence par rappeler que l'amitié afghano-soviétique est « faite pour durer dans le temps » avant de dresser un parallèle avec la guerre du Vietnam durant laquelle, souligne-til, nul n'a pu accuser le Vietnam de faire appel à des troupes étrangères.

Il informe Taraki, qui semblait l'ignorer, que selon des informations reçues à 11 heures le matin même la situation est en cours de " normalisation » à Hérat, grâce à l'action d'un bataillon de parachutistes appuyés par des blindés venus de Kandahar. Donc, poursuit-il, vous êtes capables de rétablir la situation ${ }^{4}$ et s’il vous faut de l'aide supplémentaire, nous vous la donnerons, mais il n'y aura pas d'intervention militaire.

Taraki remercie. Il explique que la conviction du gouvernement afghan est que les problèmes doivent d'abord être réglés politiquement avant de l'être militairement. Il se montre ensuite à nouveau un bon élève du marxisme-léninisme : « Notre révolution, dit-il, a provoqué une réaction des ennemis de classe. Elle a libéré les paysans de la domination des propriétaires et des féodaux, et d'autres mesures ont assuré l'autorité du gouvernement sur le peuple afghan ». Il se lance ensuite dans un long discours sur les forces réactionnaires et sur le soutien que reçoivent, selon lui, le gouvernement et sa personne de la part de la population. Il assure que le gouvernement afghan « ne veut pas gouverner par la force mais en gagnant la confiance du peuple par des réformes démocratiques en faveur des classes laborieuses ".

Ustinov, pragmatique, explique que des hélicoptères seront mis à la disposition du gouvernement afghan en juinjuillet, d'autres avant la fin de l'année. Taraki demande que des pilotes soviétiques les accompagnent. Kossyguine et Ustinov s’y opposent. Taraki les interroge alors sur la possibilité d'un « prêt » de pilotes vietnamiens ou cubains. Nouveau refus. Lorsque Taraki explique qu'il cherchera ailleurs dans le monde des pilotes, il s’attire les foudres de ses interlocuteurs. Ustinov souligne en effet que des pilotes afghans sont actuellement en cours de formation, 16 pour des avions, 13 pour des hélicoptères.

Kossyguine détaille ensuite les aides qui seront fournies : 100000 tonnes de blé, tandis que le prix du gaz afghan acheté par l'URSS passera de 23 à 37 dollars pour en assurer le paiement. Il énumère ensuite les matériels militaires qui feront l'objet de dons : 33 chars de combat BMP 1, 5 hélicoptères d'attaque MI 21, 8 MI 8T hélicoptères polyvalents, 50 transports de troupes BTR 60, 25 véhicules de reconnaissance, et des missiles sol air « Strela ». Ustinov interviendra plus tard pour préciser que des techniciens de l'armement accompagneront les livraisons d'armes.

Taraki remercie. Il demande toutefois des livraisons de blé supplémentaires. La récolte s'annonce en effet mauvaise « car les terres confisquées aux grands propriétaires n’ont pas été semées par eux et, dans certains endroits, des récoltes ont été détruites ».

Kossyguine répond que 15000 tonnes seront livrées chaque mois " pour autant que le gouvernement afghan soit en mesure d'en assurer le transport », souligne-til d'un ton grinçant. Taraki, dans l'embarras, explique alors que le Pakistan et la Turquie sont revenus sur leurs promesses de livrer respectivement 200000 tonnes et 70000 tonnes de blé. Il demande à l’URSS de lui fournir 300000 tonnes de blé. Il s’attire une réponse cinglante de Kossyguine qui lui dit que s'il avait commandé 200000 tonnes au Pakistan, c’est qu’il avait les moyens de les payer. « Trouvez ce que vous pourrez 
comme argent, et avec cette somme nous achèterons du blé aux Américains et nous le ferons acheminer ».

Après avoir tenté, sans succès, d'obtenir la construction d'une puissante radio à Kaboul pour muscler la propagande du régime, Taraki revient sur la possibilité d'obtenir des pilotes d'avions et d'hélicoptères venus d'autres pays socialistes. La question agace manifestement Kossyguine qui répète qu’il n’en est pas question.

Taraki, prend encore la parole. Il explique qu'il rendra compte à son propre Politburo de ces entretiens. Il espère une dernière fois arracher la promesse d'une intervention directe : «Devrai-je leur dire que l'URSS apportera seulement ${ }^{5}$ une aide politique et autre ? ». Kossyguine confirme et conclut en lui disant que tout ce qui a été promis lui sera confirmé par Léonid Brejnev avec lequel il va maintenant s’entretenir brièvement.

\section{L'élimination de Taraki}

Trois mois plus tard, Gromyko, Andropov, Ustinov et Ponomarev présentent un rapport au Politburo sur la dégradation de la situation en Afghanistan. Celle-ci, expliquent-ils, est due à une économie arriérée et à la faiblesse du parti communiste. Ils soulignent que le pouvoir se concentre entre les mains de Taraki et d'Amin, qui accumulent les erreurs et « violent la légalité ».

Pour aider l’armée afghane combattant les « contrerévolutionnaires », ils préconisent d'envoyer en Afghanistan, « un général expérimenté » et un groupe d’officiers soviétiques qui agiraient au sein des unités afghanes. Pour protéger les forces aériennes soviétiques de la base de Begram, ils proposent d'envoyer un bataillon de troupes parachutistes déguisées en personnel de maintenance. Enfin, pour la sécurité de l'ambassade d'URSS à Kaboul, ils envisagent d'y affecter un détachement de 125 à 150 hommes du KGB, présentés comme du personnel de l'ambassade. Ces propositions sont entérinées.

Le 14 septembre, Amin fait arrêter et assassiner Taraki, décrète l'arrestation de ses partisans au sein du gouvernement ${ }^{6}$, et s'installe au pouvoir.

Le 4 octobre, lors d'un entretien avec Erich Honecker, président de la RDA, Brejnev fait part de son inquiétude sur la situation en Afghanistan. Il décrit Amin comme un homme trop autoritaire usant de méthodes brutales : « Nous ne sommes pas satisfaits de ses méthodes et de son action ». La sanction s'annonce.

\section{Vers l'intervention militaire}

Le 29 octobre, le quatuor cité plus haut présente un nouveau rapport au Comité central. À la suite du coup d'État du 14 septembre, soulignent-ils, en dépit de la libération de prisonniers, la vague de répression s'est

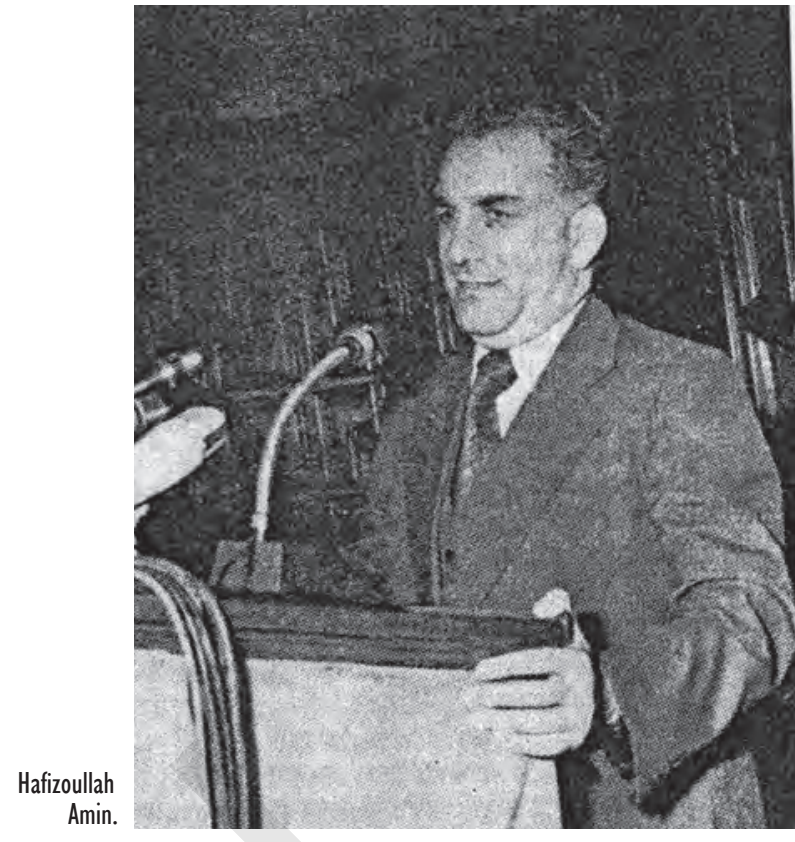

élargie. Ils révèlent que l'exécution de quatre membres du politburo afghan est programmée, qu’Amin place ses proches, y compris des membres de sa famille, dans les organes d'État. Ils ajoutent que des signes d'un changement de ligne politique sont perçus comme une « politique d'équilibre » qui « contente Washington ».

Face à ce qui est perçu comme une menace avec une possible victoire de la " contre révolution », le quatuor propose de continuer à aider Amin sans lui laisser penser qu'on ne le croit pas afin de s'efforcer de découvrir ses intentions réelles. Dans le cas où il apparaîtrait que la politique d'Amin évoluerait dans une direction antisoviétique, il conviendrait de «faire des propositions supplémentaires sur les mesures à prendre ».

Au début décembre 1979, Andropov adresse un mémorandum à Brejnev. Il souligne qu’ Amin se « tournerait vers l'Ouest » car il a des contacts secrets avec des Américains ${ }^{7}$. Il aurait assuré à des chefs tribaux qu’il se détacherait de l'URSS et établirait une politique de neutralité.

Andropov explique ensuite que Babrak Karmal, exilé comme ambassadeur en Tchécoslovaquie, et Assadollah Sarwari, réfugié à l'ambassade d'URSS à Kaboul, ont un plan pour s'opposer à Amin et créer un nouveau parti. Pressentant la menace, Amin, disent-ils, a procédé à des arrestations préventives et a fait exécuter 300 personnes. Dans ces conditions Karmal et Sarwari, sans changer leurs plans, ont posé la question d'une assistance - y compris militaire - à leur projet. Andropov dit qu'il y a deux bataillons soviétiques à Kaboul et qu'en cas de soutien au projet de Karmal, il conviendrait, par précaution, de masser des troupes à la frontière. Il conclut en assurant qu'il faut conserver les gains de la révolution d'avril 1978, donc renforcer les 


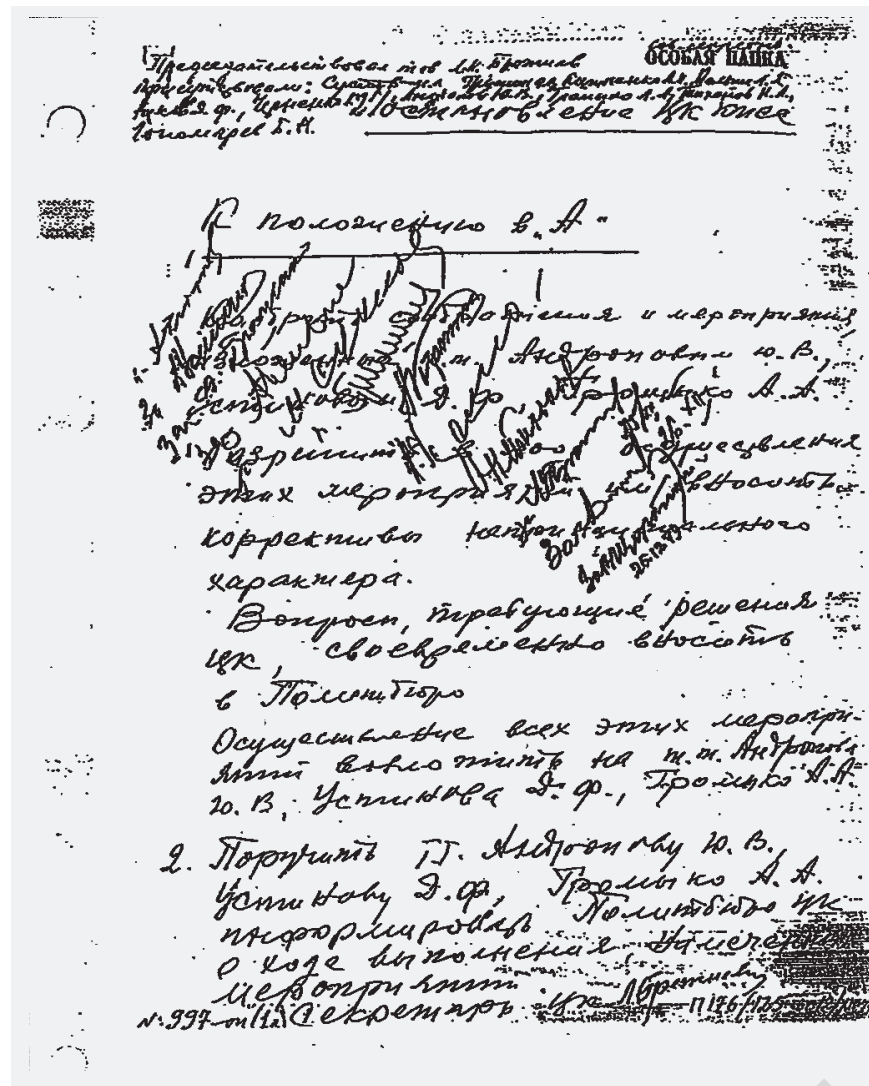

positions de l'URSS en Afghanistan.

Le 8 décembre, une réunion a lieu dans le bureau de Brejnev, en présence d'Alexandre Liakovski, major général, de l'état-major des armées. Y participent Andropov, Gromyko, Souslov et Ustinov. Les services de renseignement soviétiques ont acquis la certitude que le résident de la CIA à Ankara essaie de mettre sur pied un nouveau « grand empire ottoman » qui inclurait les républiques soviétiques d'Asie centrale. Selon eux, cela fait courir le risque de voir les États-Unis installer des fusées Pershing en Afghanistan dont des régions entières pourraient être annexées au Pakistan... La réunion se conclut sur la nécessité de destituer Amin et de déployer des troupes soviétiques en Afghanistan.

Le 10 décembre, le ministre de la Défense, Ustinov, informe Ogarkov, chef d'état-major des armées, de la décision d'introduire 75 à 80000 hommes sur le territoire afghan. Ogarkov assure que cela sera insuffisant pour normaliser la situation, qu'il s’y oppose. Ustinov réplique sèchement qu'Ogarkov n'est pas là pour discuter les décisions du Politburo mais pour exécuter ses ordres.

Le même jour, une réunion a lieu avec les membres du comité restreint dans le bureau de Brejnev en présence d'Ogarkov. Celui-ci tente de convaincre ses interlocuteurs qu'ils font fausse route. En vain. Le soir même, Ustinov convoque les principaux chefs militaires et leur annonce la décision prise. Dès le 10, des troupes sont dirigées vers le Turkestan.

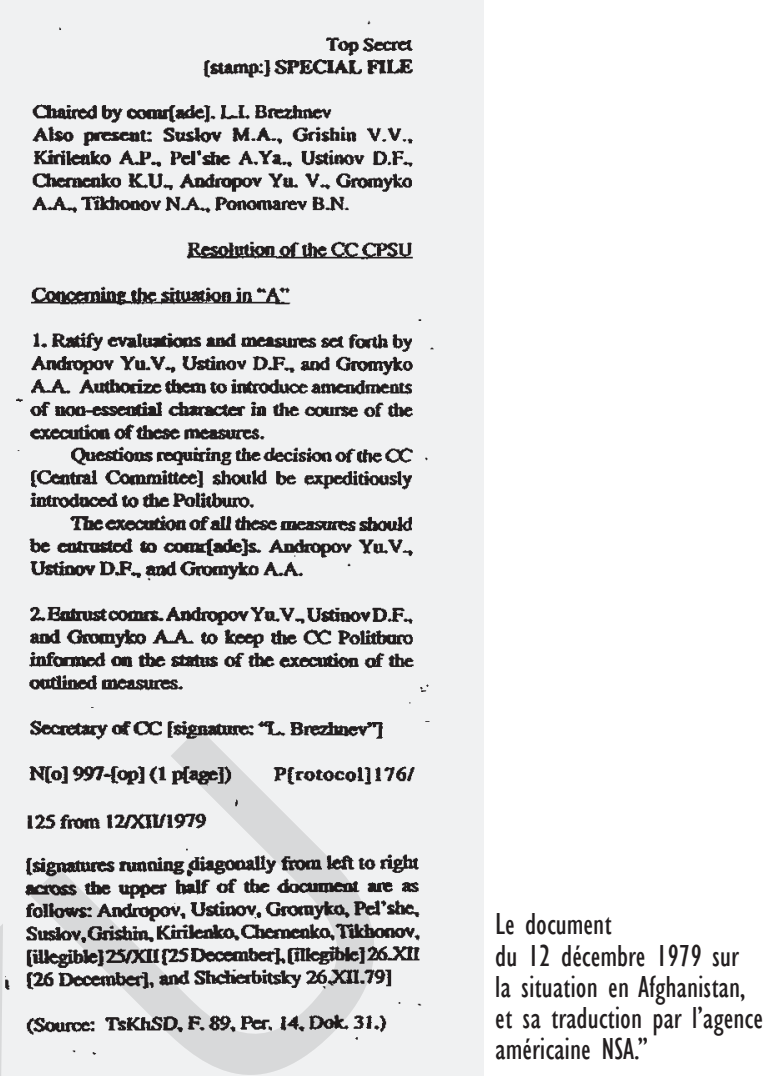

Au matin du 12 décembre, Ustinov reçoit un rapport du chef du KGB en Afghanistan. À l'initiative d'Ustinov, Andropov et Gromyko, son contenu lève les dernières réticences du Politburo, présidé par Brejnev, sur la nécessité d’une intervention. Kossyguine, défavorable à l'intervention militaire, n'assiste pas à cette réunion. Un procès verbal secret et manuscrit est rédigé par Tchernenko ; il est signé par les onze présents sur les seize membres du Politburo. Il sera ultérieurement conservé dans un coffre. Les ordres seront donnés oralement. Il n’y aura pas de décret du Præsidium.

La suite est connue.

1- The National Security Archive. The September 11th Sourcebooks, vol. II. Afghanistan : Lessons from the Last War. The Soviet experience in Afghanistan. Russian documents and Memoirs. (Documents 1 à 9 inclus).

2- Sur le soulèvement de Hérat, voir Mohammed Sediq Farhang, Afghanistan, les cinq derniers siècles, tome 2 (1919-1979), Ceredaf, pp. 324-328. Farhang évoque 5000 à 45000 tués, le « Conseil culturel du djihad de l'islam » avançant le chiffre de 25000 morts.

3- Léonid Brejnev était malade et affaibli. Il devait mourir le 10 novembre 1982. Youri Andropov lui succèdera.

4- Il est pourtant avéré que des avions soviétiques sont intervenus pour réduire la rébellion.

5- C'est nous qui soulignons.

6- Watandjar, Sarwari (qui se réfugiera à l'ambassade d'URSS), Golabzoï, Mazdouryar. En réalité Taraki n’aurait été exécuté que le 8 ou le 9 octobre (Farhang, ouvr. cité, p. 348).

7- Selon Mohammed Sediq Farhang (ouvr. cité, p. 354) les Américains n'avaient guère confiance dans Amin et n'accordèrent que peu d'attention à ses manœuvres d'approche. 


\title{
Le primat de la culture dans la relation franco-afghane
}

\author{
Par Régis KOETSCHET*
}

\begin{abstract}
Quelle est la vraie nature de la relation franco-afghane ? Certains ont tenté de déprécier la dimension culturelle du lien entre la France et l'Afghanistan. Cela ne rapportait rien. Régis Koetschet, à l'inverse, souligne le caractère fondamental de ce qu'on pourrait appeler la connivence spirituelle entre les deux pays.
\end{abstract}

Le 1er juin 1965, le général de Gaulle accueillait à Orly le roi Zaher Chah en visite officielle en France par ces mots : « Si la situation de l'Afghanistan a pu nous paraître lointaine, nous n'en étions pas moins proches de lui par la culture et le sentiment ». Le soir, lors du diner à l'Elysée, il prolongeait sa pensée : «Bien entendu, c’est par la culture que le mouvement a commencé, car tout procède de l'esprit. Aussi, sous la conduite d'Alfred Foucher, un groupe de savants archéologues de chez nous fut tout de suite attiré par ce pays rempli de vestiges du passé. (...) En même temps, nos universitaires, nos juristes, nos médecins prenaient contact avec les élites afghanes et celles-ci faisaient à la France l'honneur d’utiliser sa langue, de pénétrer son génie et d'accueillir son enseignement ».

En mars 1967, il y a juste cinquante ans, paraissait le livre de Joseph Kessel « les Cavaliers ». Ce fut un grand succès de librairie mais aussi une séquence importante dans la vie et l'œuvre de Jef qui souhaitait par ce roman « rendre » à l'Afghanistan tout ce que ce pays lui avait « donné ». Cette histoire de tchopendoz demeure emblématique de l'image que le public français s’est longtemps fait de l’Afghanistan. Elle a été récemment revivifiée par la pièce éponyme montée par Eric Bouvron et Anne Bourgeois (prix du public au festival off d’Avignon en 2014) et par le livre de Louis Meunier (« les cavaliers afghans »).

* Ancien ambassadeur de France en Afghanistan. Président de MADERA.
Ces deux balises, pour décalées qu'elles apparaissent alors que la relation bilatérale évoque surtout aujourd'hui l'obscurantisme et le terrorisme contre lesquels auront été engagées les forces françaises et qui, le 11 décembre 2014, dans un attentat, viseront l'Institut français de Kaboul, témoignent d'une spécificité prégnante et féconde. La culture, l'écriture, le patrimoine, la connaissance et l'image sont « au cœur » de la relation entre la France et l'Afghanistan. Ils en constituent l'inspirateur et le moteur, ayant souvent eu un temps d'avance sur la diplomatie.

\section{Le socle culturel}

Ce fondement résulte d'un choix afghan. Celui du roi Amânullâh désireux de confier à la France la formation d'une partie de ses élites puis la protection de son patrimoine. Paris répond avec prudence mais favorablement. Une mission d'identification est confiée en 1922 à Alfred Foucher, instruit de rallier Kaboul à partir de la Perse, sans doute pour ne pas froisser les Britanniques. Elle conduira à la mise en place d'une structure éducative, le collège Amaniyeh, et à la création de la Délégation archéologique française en Afghanistan (DAFA). Elle ouvrira la voie à la venue de Maurice Fouchet comme premier représentant diplomatique français. Ce dernier a relaté cette expérience dans un ouvrage posthume («Notes sur l'Afghanistan ») préfacé par Joseph Hackin, directeur de la DAFA qui rappelle que le chef de la légation, « et à son exemple, ses collaborateurs, 


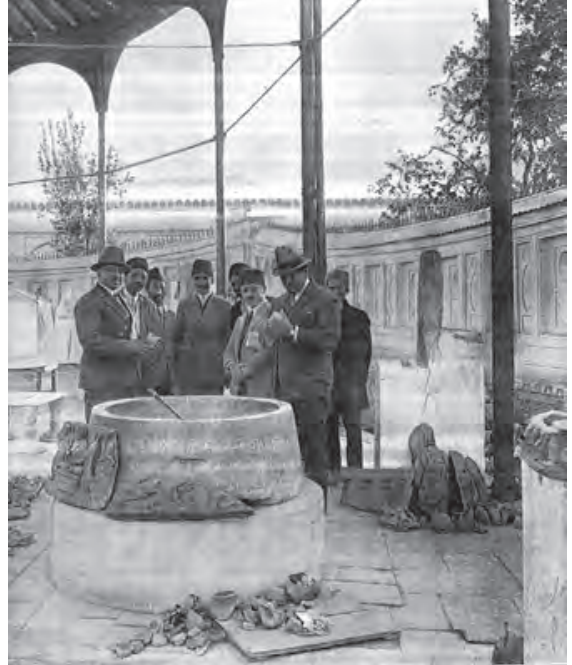

Le roi Amânullâh au musée de Kaboul en 1929 (extrait de L'lllustration)

savaient dispenser aux œuvres françaises le meilleur de leur activité ».

La littérature n'est jamais loin et à Paris, l'homologue de Fouchet, Mahmoud Tarzi, traduisait Jules Verne...

Comme l'a joliment écrit Gilles Rossignol, dans son avant-propos aux Actes du colloque du CEREDAF « 90 ans de relations France-Afghanistan 1922-2012 Histoire et perspectives ", il apparaît que l'archéologie a constitué la " pierre fondatrice » de cette relation, l'éducation (lycées Esteqlâl et Malâlaï) en formant son « architecture ». En investissant les secteurs du patrimoine et de la formation, la France montrait aux yeux des Afghans qu'elle n’avait pas d' " agenda caché ». «Il me paraît certain que notre éloignement géographique et la bienheureuse absence de tout intérêt politique dans ce pays nous valent la confiance générale » écrira Alfred Foucher. Cette perception, globalement, perdure.

Ce contenu culturel va " imprimer » la relation politique. La revue « l'Illustration » publiera deux photographies pleine page montrant le roi Amânullâh « prenant sa leçon de français » (22 novembre 1924) et « examinant, au musée de Kaboul, les objets recueillis par la mission française au cours de ses fouilles » (29 décembre 1928). En 1929, lors des troubles fomentés par le Batche saqao et qui vont conduire à la chute d'Amânullâh, la communauté diplomatique est évacuée. La gestion de la légation française est confiée à l'archéologue Joseph Hackin. La journaliste Andrée Viollis, arrivée à Kaboul début octobre, raconte dans son ouvrage « Tourmente sur l'Afghanistan » les premiers jours de la reprise en main par Nâder Chah. Elle conclut en soulignant la portée de l'« entente archéologique » franco-afghane qui a ajouté des « joyaux inconnus au patrimoine artistique du monde ». En Afghanistan, « la France, nous le répétons, a donc dans le domaine moral et spirituel qui est plus proprement le sien, un grand rôle à jouer, à poursuivre ».
En 1940, la culture rejoindra une nouvelle fois l'Histoire. Joseph Hackin, ayant eu connaissance de l'Appel du 18 juin, et devant un poste diplomatique français attentiste, se rend à la légation britannique. Il fait envoyer un message à de Gaulle marquant son ralliement ainsi que celui de son épouse Ria et de Jean Carl, architecte à la DAFA. Ils rejoignent immédiatement Londres tout comme André Beaudoin, professeur au lycée. Les Hackin périront en mer en février 1941 en route pour une mission en Asie et seront faits Compagnons de la libération. André Beaudoin dirigera l'Ecole des cadets de la France Libre au Royaume Uni.

D’autres personnalités culturelles liées à l'Afghanistan (Gabriel Monod Hertzen, professeur à l'université de Kaboul, Daniel Schlumberger futur directeur de la DAFA et ses collègues Raoul Curiel et Jean-Claude Gardin) rejoindront les comités de la France Libre ou radio Brazzaville. On peut aussi citer Charles Hinstin, jeune résistant, dont Kessel a raconté l'amitié dans « le zombie » (« Tous n'étaient pas des anges ») et qui repose au cimetière pour étrangers de Kaboul.

\section{Une amitié fondée sur la culture}

Une relation bilatérale, forte et bienveillante, va se développer en articulant des composantes politiques et de coopération mais aussi, ce qui est plus inhabituel, en se nourrissant de références culturelles et en puisant même dans le registre de l' " imaginaire ». C'est ce que relèvera Renaud Donnedieu de Vabres, le 24 mai 2004 à Kaboul, pour la première visite en Afghanistan d'un ministre français de la culture : « Votre pays fait partie de l'imaginaire de la France, qui a toujours rêvé de l'Afghanistan, grâce à ces passeurs de rêves que sont Joseph Kessel ou André Malraux (...). La solide amitié entre nos deux pays s'est elle-même construite sur la culture ».

\section{Avant 1950}

Jusqu’à la deuxième guerre mondiale, alors que la relation politique est peu étoffée (dans sa préface à la récente réédition du livre de René Dollot, Gilles Rossignol rappelle à cet égard la « maladresse » des autorités françaises à l'égard des ministres afghans en visite officielle), c'est clairement la coopération (éducation, agriculture, patrimoine) mais aussi la culture qui occupent tout l'espace bilatéral.

A cette époque, l'Afghanistan suscite une curiosité intellectuelle. « l’ Illustration », dans sa livraison 
du 9 février 1929 publie pas moins de quatre pages richement illustrées sur les fouilles de Hadda sous la signature de son promoteur, l’archéologue Barthoux. Le même mois, sous le titre « une grande découverte artistique », la revue novatrice « Vu » de Lucien Vogel publie une série de photographies de Germaine Krull sur cette statuaire. Ces deux articles correspondent à l'ouverture par le Président de la République d'une nouvelle salle au musée Guimet, dédiée au Gandhara. Joseph Hackin, à l'aide de grandes photographies, y fait déjà le rapprochement avec le sourire gothique de l'archange de Reims. C'est dans ce contexte qu'André et Clara Malraux se rendront à l'été 1930 en Afghanistan. Outre un matériau littéraire (« Les noyers de 1'Altenburg " qui s’inspirent en partie de ce voyage seront publiés en 1945), ils en ramènent des statues qui seront présentées lors de deux expositions à la galerie de la NRF de Gaston Gallimard.

L’année suivante a lieu la "Croisière jaune » qui du 19 mai au 20 juin traverse l'Afghanistan. Plusieurs hommes de culture (Joseph Hackin, le peintre Alexandre Iacovleff, le cinéaste André Sauvage et, pour la partie chinoise, le père Teilhard de Chardin et le naturaliste André Reymond) font partie de cette expédition automobile qui conjugue intérêts commerciaux, influence et découverte culturelle. S'agissant de l'Afghanistan, ses résultats seront présentés à Paris dans les salons Citroën par l'historien René Grousset.

Si les Malraux sont bien allés en Afghanistan, d'autres écrivains à fort tirage vont s'intéresser à ce pays en restant sur ses abords indiens. Francis de Croisset dans « Nous avons fait un beau voyage » (1925), Maurice Dekobra dans « les Tigres parfumés » (1929) et dans « le Sphinx a parlé » (1930), Pierre Benoit dans " Bethsabée » (1938) traitent de façon romanesque de la présence britannique face à l'Afghanistan. Une littérature qui véhicule de vigoureux clichés sur les Afghans. Ces romanciers s'inscrivent dans la lignée de prestigieux anciens qui, au siècle précédent, ont écrit sur l'Afghanistan ou ses habitants sans y avoir mis non plus les pieds. Arthur de Gobineau (les Nouvelles asiatiques, Trois ans en Asie) qui voit dans les Afghans un " peuple singulièrement romanesque ", Pierre Loti, Jules Verne. On réservera une mention particulière à Octave Mirbeau. Ses « lettres d’Inde » écrites depuis sa chaumière du Perche sont un régal.

La lettre $n^{\circ} 7$ datée de Péchaver 8 mars 1885 est intitulée « l’Afghan ». « Les Afghans ! Voilà un mot qui retentit dans l'Inde comme un cri de terreur ; ce peuple vaincu, il y a deux ans, fait peur à ses vainqueurs aujourd'hui, (...) ce peuple dont les destinées sont si importantes pour la paix dans le monde. »

A cette thématique de l'Afghan « à la grande barbe noire, à l'œil cruel et au nez d'épervier » (Mirbeau) va s'ajouter une veine onirique porteuse d'imaginaire, celle d’un pays magique et interdit, à la géographie de « bout du monde », dernière marche avant « les horizons perdus » pour reprendre le titre du best-seller de James Hilton.

Cette lecture très stimulante aura plusieurs déclinaisons. En 1908, Alfred Kubin installe son « Royaume rêvé » (« Die andere Seite ») à la frontière orientale de l’Afghanistan. Par la suite, d'aucuns situeront le Farghestan du « Rivage des Syrtes » dans cette même aire géographique. Le musée Guimet n’hésitera pas en 2002 à « sauter le pas » reproduisant un passage du livre de Julien Gracq dans le catalogue de l'exposition sur « une histoire millénaire ». «Les invasions qui l’ont balayé de façon presque continue depuis les temps antiques - en dernier lieu l'invasion mongole - font de sa population un sable mouvant où chaque vague à peine formée s'est vue recouverte et effacée par une autre, de sa civilisation une mosaïque barbare où le raffinement extrême de l'Orient côtoie la sauvagerie des nomades ».

Auteur de livres d'aventure, André Armandy publie en 1926 dans la collection des « romans mystérieux » « Terre de suspicion » qui se passe en Afghanistan et où une montagne magnétique attire au sol les avions.

Les surréalistes vont également jouer avec ce « mystère » afghan. Le lévrier, du fait de son allure excentrique, sera leur guide. Picasso en aura trois successivement (Kazbek, le plus célèbre, puis Kaboul et Sauterelle). On le retrouve dans des tableaux du maître ou sur des photos de Brassai et de Man Ray. Il inspirera « le chien » de Giacometti. Il figure dans le tableau de Salvador Dali « Afghan invisible avec apparition sur la plage du visage de Garcia Lorca en forme de compotier aux trois figues » (1938). Dans le premier numéro de la revue «Eléments » de Michel Leiris (1930) René Grousset publie un article intitulé « un cas de régression vers les arts «barbares » : la statuaire du Kafiristan ». Sur le plan de l'écriture, même inspiration afghane décalée. Max Jacob nomme Kaboul 1er son roi des Balbriges. Raymond Queneau, en 1936 dans son autoportrait fictif (« les derniers jours ») fait débarquer le personnage « rue de Caboul » - une adresse supposée éveiller son inspiration littéraire - (« Il tenta de s'exalter en pensant qu'il logeait rue de Caboul et que cette ville est la capitale de l'Afghanistan, mais sans y réussir »). L'un de ses disciples de l'OULIPO (OUvroir de LIttérature POtentielle), l’écrivain américain Harry Mathews écrira en 1978 « les verts champs de moutarde de l’Afghanistan » traduit par Georges Perec. L'écrivain hongrois Tibor Tardos, ami d'André Breton, publiera en 1982, c'est-à-dire pendant l'intervention soviétique, un roman dont le titre est « lévrier afghan », une folle histoire de réseau ferroviaire dans un cadre totalitaire.

Cette approche fantasmatique quelque part perdure comme en témoigne le très beau film « Ni le ciel ni la terre » de Clément Cogitore (2015). Des soldats français disparaissent comme aspirés par la terre afghane. 


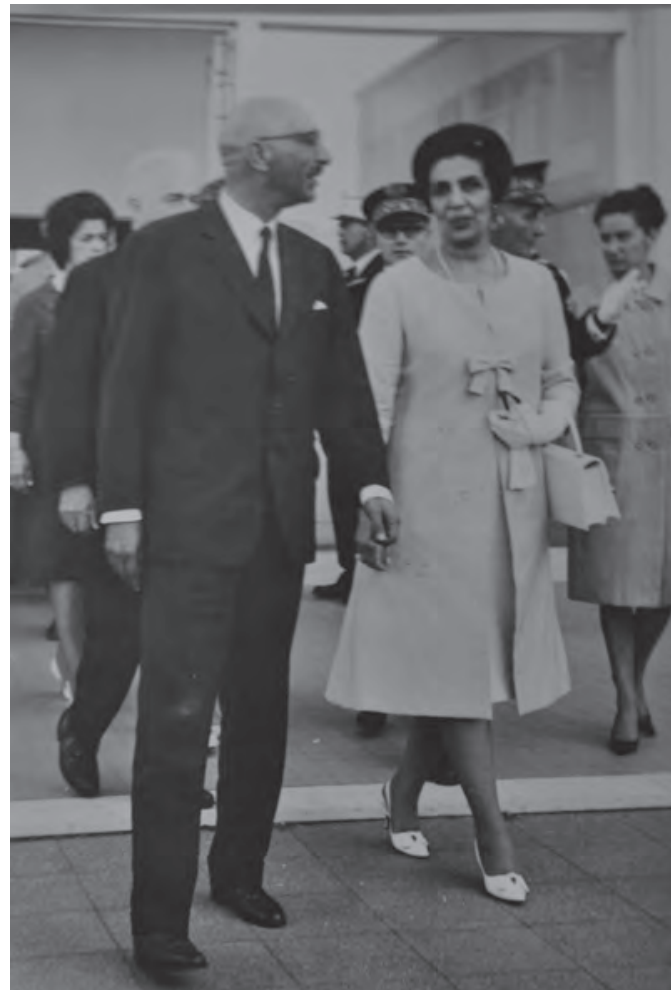

Le roi Zaher Chah et la reine Soraya en visite à Paris.

\section{Joseph Kessel Les cavaliers}

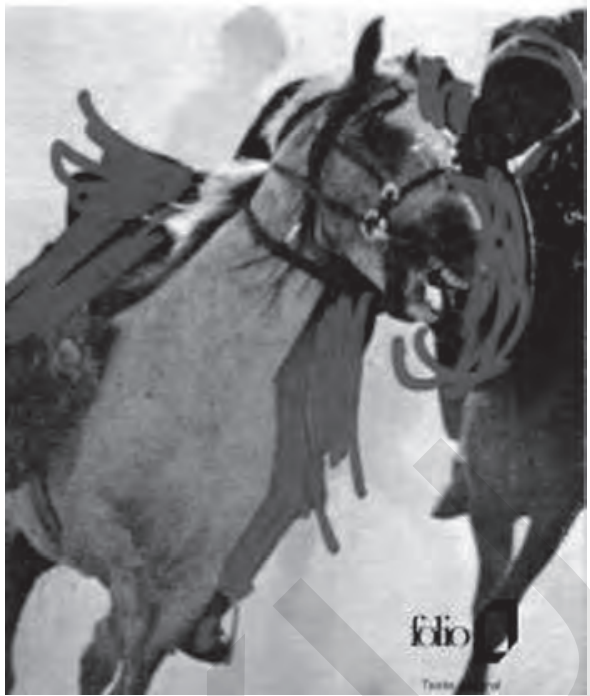

Les Cavaliers, de Joseph Kessel

dans ce contexte que s'inscrit la visite officielle du souverain afghan en 1965, celle à Paris en avril 1967 du premier Ministre Muhammad Hâchem Maiwandal et celle en Afghanistan, en mai 1968, de Georges Pompidou. Le contenu « culturel » de ces échanges sera important. Zaher Chah visitera le musée Guimet, Pompidou ira sur les sites archéologiques d’Äi Khanoum et Bâmyân et posera la première pierre du nouveau lycée Esteqlâl.

Trois artistes français vont littéralement « incarner » pendant cette période la relation entre la France et l'Afghanistan : le romancier Joseph Kessel et le couple de photographes Roland et Sabrina Michaud.

Enfin, dans les années 30, l'Afghanistan, pour les intellectuels communistes français comme Paul Nizan ou Paul Vaillant Couturier, apparaît ausscomme le parfait contre-exemple du progrès socialiste. L'un et l'autre se rendent au Tadjikistan sur la frontière afghane. « Nous étions au pied d'une des grandes lignes de partage des eaux du monde et elle ne séparait pas seulement des versants, des bassins de drainage tournés vers la mer d'Aral, la Sibérie et de l'autre côté des montagnes, vers l'Inde, l'Afghanistan, elle séparait aussi des mondes politiques, des mondes humains " écrit l'auteur d' " Aden-Arabie » dans « sindobod toçikiston ».

A la veille de la seconde guerre mondiale et alors que la relation diplomatique est modeste, l’Afghanistan fait, dans la perception française, le grand écart entre Gandhara (« la suprême sagesse par la suprême beauté » pour Malraux), mythique « berceau » himalayen et violence tribale.

\section{La période 50-80}

La deuxième phase, qui s’étend des années 50 à l'intervention soviétique de décembre 1979, va voir la relation d'amitié se renforcer tant sur le plan diplomatique que culturel.

Le 27 janvier 1964, la France reconnaît la Chine populaire. Dans sa conférence de presse du 31 janvier, le général de Gaulle citera tous les pays du voisinage de la Chine, dont l'Afghanistan, montrant bien que s'ouvre un nouveau champ pour la diplomatie française. C'est
Kessel a raconté dans « le Jeu du roi » son arrivée à Kaboul en 1956, en compagnie de Pierre Schoendorffer et de Raoul Coutard, pour y tourner « la Passe du diable », un film dont le scénario servira de trame dix ans plus tard au roman « les Cavaliers ». Jeff ressentira immédiatement une profonde empathie pour l'Afghanistan, son peuple et ses paysages. Il y retournera à plusieurs reprises (cf le beau reportage d'Igor Barrère de 1967 « Voyage en Afghanistan le désert-la montagne » diffusé par l'INA) pour, selon son biographe Yves Courrière, « boucler l'aventure d'une vie ». L'ami Georges Walter qui dans «le Livre interdit » raconte les derniers mois du romancier revient sur cette relation à l'Afghanistan, « un bain de Jouvence ». Chaque fin de semaine à Averne, Kessel retrouve sa mélancolie afghane. « Dans la maison normande, rustique et usagée, la grande pièce commune au sol carrelé avait une longue table en chêne, un vieux divan délabré, une table basse recouverte d'une douzaine de figurines de bronze - des cavaliers du bouzkachi offerts jadis par Zâher Châh ». Schoendorffer reviendra pour sa part à Kaboul en 2005, invité par les parachutistes français de l'ISAF.

En 1970, période de "summer of love » et de développement du tourisme en Afghanistan (les Français étant parmi les plus nombreux) est édité le premier « beau » livre sur l'Afghanistan dans la collection « Hachette rêves et réalités » (ça ne s'invente pas) texte de Kessel, photos des Michaud. En 1977, les Michaud publient « Caravanes de Tartarie », premier livre sous cette forme iconographique avec un tirage important qui sera 


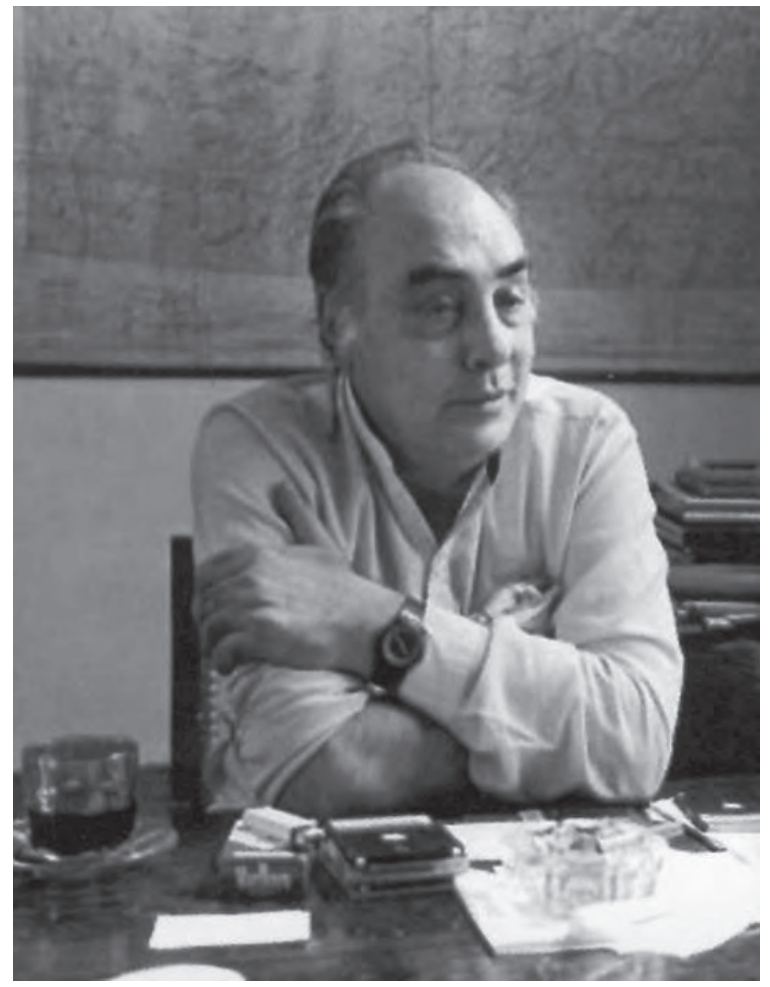

Sayed Bahoddin Madjrouh, philosophe et poète.

épuisé en trois mois. Suivront notamment « Mémoires de l'Afghanistan » (1980), « l'Orient dans un miroir » préfacé par Nadjm Oud dine Bammate, « les Cavaliers » (1988), « Faïences d'azur » avec un texte de Michael Barry (1995), «Afghanistan » dédié au « peuple afghan qui nous a ouvert l'œil du cœur », avec une préface d'André Velter (2002). Zaher Chah dira aux Michaud qu'ils ont fait redécouvrir aux Afghans leur culture.

La relation diplomatique se love avec aisance dans cet environnement esthétique, riche de mots (« Tout ce qui s’étendait entre la Perse et la Chine, entre les Arabes et les Mongols me faisait tourner la tête (...). Nous découvrions un monde dont nous n'avions aucune idée et dont seuls Gobineau, puis Malraux, et les Mille et Une Nuits, bien sûr, nous avaient dit quelques mots » relève Jean d'Ormesson dans « une Fête en larmes ») et d'images (la photographie de Marc Riboud prise en 1955 à la passe de Khyber - un cycliste enturbanné et un panneau montrant la piste pour les voitures et celle pour les chameaux - est devenue culte, Régis Debray vient de la choisir pour la couverture de ses « Carnets de route écrits littéraires » (2016).

\section{L'invasion soviétique}

L'indignation française à l'intervention soviétique de 1979 conjugue de manière assez fusionnelle l'humanitaire et la culture.

Parmi d'autres, plusieurs personnalités vont inspirer cet engagement.
Sayed Bahoddin Madjrouh, philosophe et poète (Ego-Monstre), ancien doyen de la faculté des lettres de Kaboul. En février 1980, il se réfugie à Peshawar et crée l'Afghan Information Centre. Jusqu'à son assassinat en février 1988, inlassablement il témoigne, recueille, conseille, diffuse, encourage avec son inoubliable « rire ».

Olivier Roy dans «En quête de l'Orient perdu » (2014) raconte son cheminement personnel, de la philosophie au terrain afghan et retour. Pendant toute cette période de la résistance, une réflexion majeure sur " l’islam et la modernité politique » avant de constater " l’échec de l’islam politique », et un écho porté vers les milieux décisionnels tant en Europe qu’outre atlantique.

Enfin, comment ne pas citer deux acteurs, disparus trop tôt, emblématiques de cette approche où l'action se conjugue avec des formes d'expression artistique : Christophe de Ponfilly fera littéralement connaître la résistance afghane avec son reportage réalisé dans le Pandjchir " une vallée contre un empire » ; il popularisera la figure du commandant Massoud ; jusqu'au bout il aura recours au livre (« Lettre ouverte à Joseph Kessel sur l'Afghanistan ») et à l'image (« l'Etoile du soldat ») pour exprimer son affection d'écorché aux Afghans. Didier Lefèvre est « le Photographe », l'auteur avec Emmanuel Guibert et Frédéric Lemercier, de cette bande dessinée/reportage photographique qui, en trois volumes, raconte d'une façon magistrale les tribulations de cet homme d'image dans une mission humanitaire de MSF. Sous le nom d'Ahmadjan, il en effectuera huit. Didier Lefèvre est par ailleurs l'auteur d'un très beau livre sur l’Afghanistan « le pays des citrons doux et des oranges amères » (2003).

La diplomatie suit le mouvement, rendant avec discrétion cet élan humanitaire possible. Il est d'ailleurs intéressant de noter que, comme si ça allait de soi, le politique mettra ses pas dans ceux d'hommes de culture. Régis Debray, alors conseiller à la Présidence de la République, viendra à Peshawar à la rencontre des partis de la Résistance. Jean-François Deniau, académicien et homme politique, passera symboliquement la fin d'année 1985 « à l’intérieur », dans la Kunar, pour marquer sa solidarité avec le peuple afghan (« Deux heures après minuit »).

\section{Sécurité et culture}

Avec la chute des Tâlebân débute une nouvelle phase dans laquelle la France entend prendre sa part à la sécurisation et la reconstruction de l'Afghanistan.

Elle s’ouvre symboliquement par un « Rapport » demandé par le Premier Ministre à Bernard-Henry Lévy comme si, là encore, le politique passait par l’’intellectuel.

En visite à Kaboul, le 3 septembre 2002, Dominique 


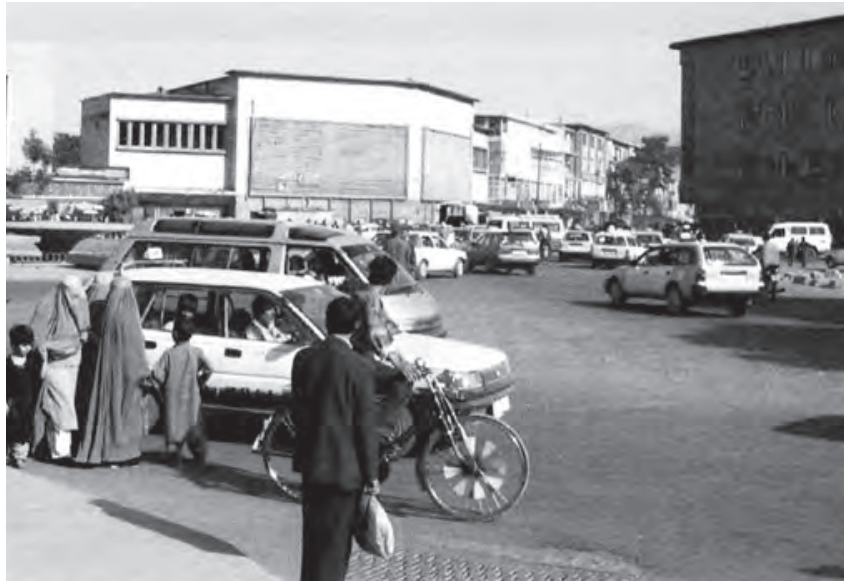

Kaboul, le cinéma Ariana, réhabilité à partir de 2002.

de Villepin rend hommage à la « vieille fraternité entre nos deux peuples ; vieux dialogue culturel qui a permis de maintenir, même aux heures les plus sombres du régime afghan, un lien précieux entre les partisans de la liberté de nos deux pays ».

Le monde de la culture sera fortement associé à la reprise de la coopération (France Culture lance en 2002 une opération « redonnons vie aux lycées francoafghans de Kaboul » qui aura un important impact ; sur une idée d' Hugues Dewavrin, le cinéma Ariana est réhabilité avec l'appui d'un collectif présidé par Claude Lelouch ; de 2002 à 2008, l' INA poursuit un programme de sauvegarde et de numérisation des archives audiovisuelles afghanes ; les Ateliers Varan, avec l'enthousiasme, la sensibilité et le savoir-faire de Séverin Blanchet, montent un projet de formation de documentaristes pour que les Afghans « se réapproprient leur histoire ». Séverin sera tué dans un attentat terroriste à Kaboul le 26 février 2010. Cet article est dédié à sa mémoire.

Culturelles, ces initiatives sont aussi très politiques car, comme le dit le président Hâmed Karzai, « il s’agit de reconstruire à la fois le présent et le passé ».

- La DAFA rouvre rapidement ; deux expositions sont organisées au musée Guimet respectivement en 2002 (une histoire millénaire) et 2006 (les trésors retrouvés). Elles portent un incontestable message politique. Les Tâlebân ont détruit les bouddhas de Bâmyân mais le patrimoine afghan reste "vivant ». S'agissant des «trésors retrouvés », il y a au moins deux enjeux politiques additionnels : accepter de « faire sortir » pour la première fois ces merveilles et ce faisant exprimer une confiance envers la France, être pour la France le premier pays à les accueillir comme les archéologues français furent les premiers à les découvrir.

- Le centre culturel français devenu l’Institut français est un lieu unique à Kaboul. Il y accueille, jusqu’à l'attaque meurtrière qui le vise, créations artistiques, expositions, débats citoyens, cours de langue.

- La France ne peut accepter de voir ce symbole de

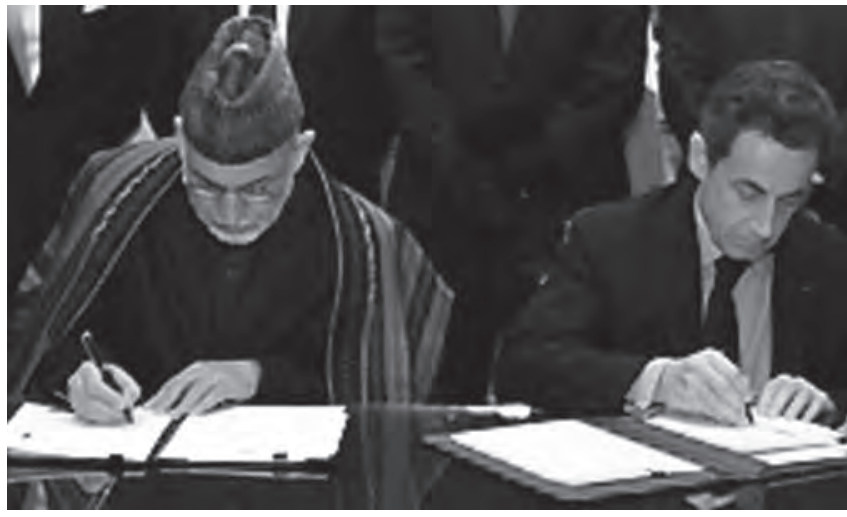

Paris, signature du Traité d'amitié et de coopération par Hâmed Karzaï et Nicolas Sarkosy.

l’amitié franco-afghane effacé. Au soir de l’attentat, le Premier Ministre publie un communiqué : «En s'attaquant à un symbole de la culture et de l'universalité des valeurs portées par la France, les terroristes montrent une nouvelle fois qu'ils véhiculent un message de haine et d'obscurantisme. (...) La France poursuivra sans faillir son action en faveur de la culture et de l'éducation en Afghanistan, condition essentielle du développement et de la sécurité de ce pays ».

- Le Traité d'amitié et de coopération signé au plus haut niveau le 27 janvier 2012 formalise dans son article 7 la place reconnue à la culture dans la relation bilatérale. "Les Parties coopèrent en vue d'encourager les échanges culturels entre les deux pays et la connaissance réciproque de leur culture. Des actions communes ont pour but de rendre la culture accessible à tous, hommes et femmes, de mettre en valeur le patrimoine culturel afghan et d'encourager la création culturelle. Les Parties coopèrent en vue de contribuer à protéger le patrimoine archéologique afghan, à renforcer l'enseignement et la recherche universitaire historiques et archéologiques et à mettre en valeur les œuvres d'art et les éléments patrimoniaux ». Le traité mentionne également le bon fonctionnement de l'IFA, de la DAFA et de l'Institut national d'archéologie.

$\mathrm{Si}$, comme le dit Karzai, en français et dans un sourire, la relation bilatérale est " romantique », on voit qu'elle s'attache aussi à faire de la culture une « arme » de progrès et de liberté.

\section{Paris-Kaboul et retour}

Depuis bientôt un siècle, la culture a donc fortement irrigué la relation bilatérale lui donnant mémoire et sens.

Dans un contexte de mondialisation, d'universalité et d'amitié sincère, cette spécificité ne saurait être à sens unique. L'attribution du prix Goncourt, en 2008, à Atiq Rahimi pour son roman écrit en français «Syngué Sabour terre de patience » est emblématique et significative du chemin parcouru depuis les premiers 


\section{CULTURE}

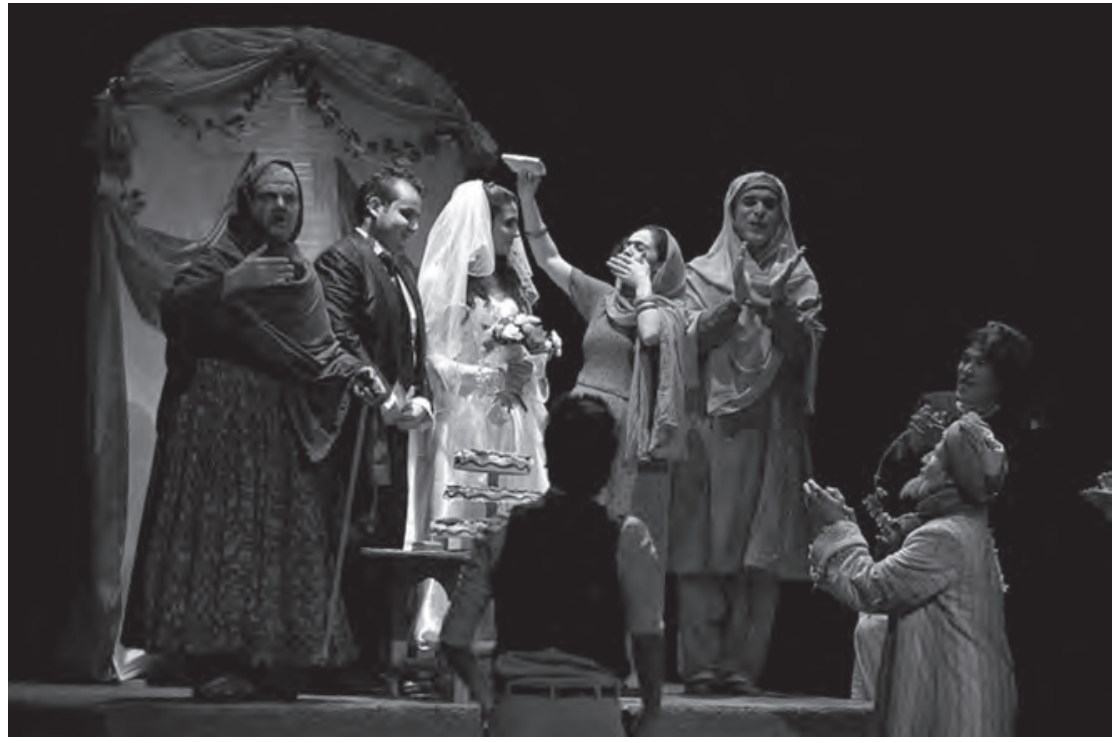

Paris, la troupe de comédiens du Théâtre Aftab.

pas d'Alfred Foucher en terre afghane. On souhaite que les créateurs afghans continuent à s’épanouir entre leur pays et la France.

Sous le titre « Kandahar », la revue « l'avant-scène cinéma » a publié en février 2016 un numéro dédié à l'Afghanistan (films qui concernent ce pays, réalisateurs qui en sont issus, liens avec la France etc...). Il y est bien sûr question de la famille Makhmalbaf mais aussi de Siddiq Barmak (Osama), d'Atiq Rahimi (Terre et cendres, Syngué Sabour) et de Barmak Akram (l'Enfant de Kaboul, Wajma). Shahrbanoo Sadat, formée au cinéma par Séverin Blanchet, vient d'obtenir un réel succès avec son premier long-métrage « Wolf and sheep ».

A la Cartoucherie de Vincennes, Ariane Mnouchkine accueille le " Théâtre Aftaab en voyage » dont elle est à l'origine depuis une mission à Kaboul en juin 2005. Cette troupe afghane joue avec un grand bonheur « la Ronde de nuit », une création collective inspirée par la migration et coproduite par le Théâtre du Soleil et celui des Amandiers avec le soutien du Ministère des Affaires étrangères et du Développement International, de deux collectivités territoriales (Région Rhône Alpes et Ville de Paris) et de la DRAC Ile de France, confirmant les articulations naturelles portées par la relation avec l’Afghanistan. Au-delà, le thème de la pièce renvoie quelque part aux propos du général de Gaulle en 1965 sur l' « honneur » qu'il y avait de voir les Afghans se pénétrer de la langue et du génie français.

Dans son message ouvrant le catalogue des « trésors retrouvés », Jacques Chirac rappelle qu' « un dialogue confiant entre les civilisations constitue pour le monde une ardente nécessité » et souligne " la place inestimable que la richesse culturelle afghane » peut avoir à cet effet.
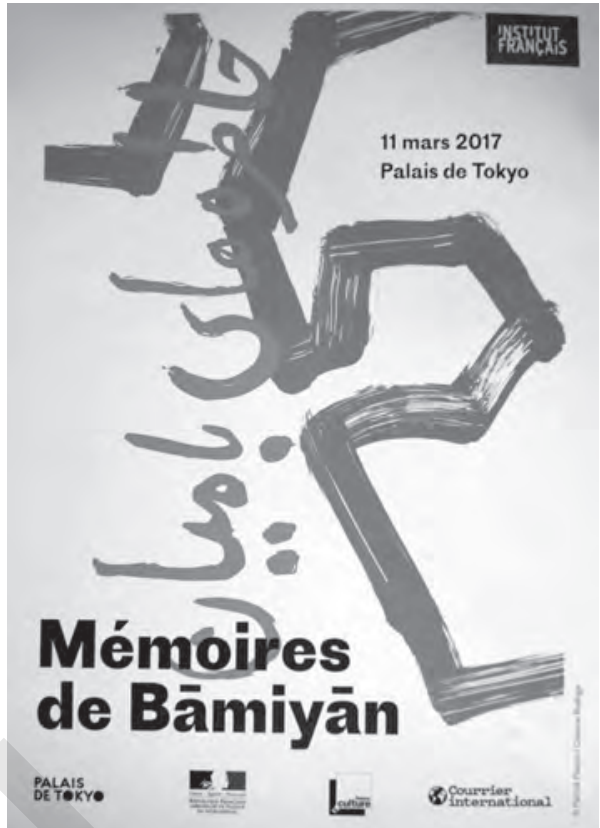

Paris, Journée de réflexion sur Bâmyân au Palais de Tokyo, le II mars 2017, affiche.

Alors que le dialogue politique semble s'être quelque peu distendu, la culture joue le rôle de vigie ardente qui lui est toujours revenu.

- le 3 décembre 2016, le président Achraf Ghani est au côté de François Hollande pour la Conférence d'Abou Dhabi sur la protection du patrimoine culturel en péril dont la France et les Emirats arabes unis ont pris l'initiative. L'Alliance internationale pour la protection du patrimoine dans les zones de conflit - fonds pour la protection et réseau de refuges pour une sauvegarde temporaire - est lancée le 20 mars 2017 dans la cour Khorsabad du Louvre. Dans son message, le président Ghani marque que « l'Afghanistan dont une partie représentative de son patrimoine - on pense souvent aux grands Bouddhas de Bâmyân et aux collections du musée de Kaboul - a été la première victime de la folie assassine et destructrice de l'obscurantisme commandité et imposé de l'extérieur, apporte son témoignage et tout son souhait de coopération pour bénéficier du soutien des nouveaux mécanismes mis en place ».

- Seize ans après la destruction des Bouddhas, l'ambassade de France à Kaboul et l'Institut français organisent en partenariat avec le Palais de Tokyo et France Culture une journée de réflexion à Paris, le 11 mars, sur Bâmyân, cet « hyper lieu » entre images et enjeux politiques.

Que « voient et nous disent les bouddhas » alors que s'ouvre en France un nouveau quinquennat que l'on espère fécond pour la relation d'amitié entre les deux pays?

1- Petit nom de Joseph Kessel (NDLR)

2- Nom afghan des cavaliers joueurs de bozkachi. 


\title{
Afghanistan Info 1980-2017
}

\author{
par Micheline CENTLIVRES-DEMONT*
}

Il faudra s'y habituer. Le "Bulletin » Afghanistan Info, publié depuis décembre 1980, ne paraîtra plus. C'était une source précieuse d'informations et surtout d'analyses, permettant de mieux percevoir les enjeux et les évolutions de la société et de la politique afghane. Micheline Centlivres-Demont, responsable de sa publication durant toutes ces années, rappelle l'histoire de ce journal et l'esprit dans lequel il était rédigé.

Le lundi 24 avril 2017 a paru le dernier numéro d'Afghanistan Info. Les 700 exemplaires sont partis pour la Suisse, la France, l’Allemagne et la GrandeBretagne, mais aussi pour l'Afghanistan, les États-Unis,

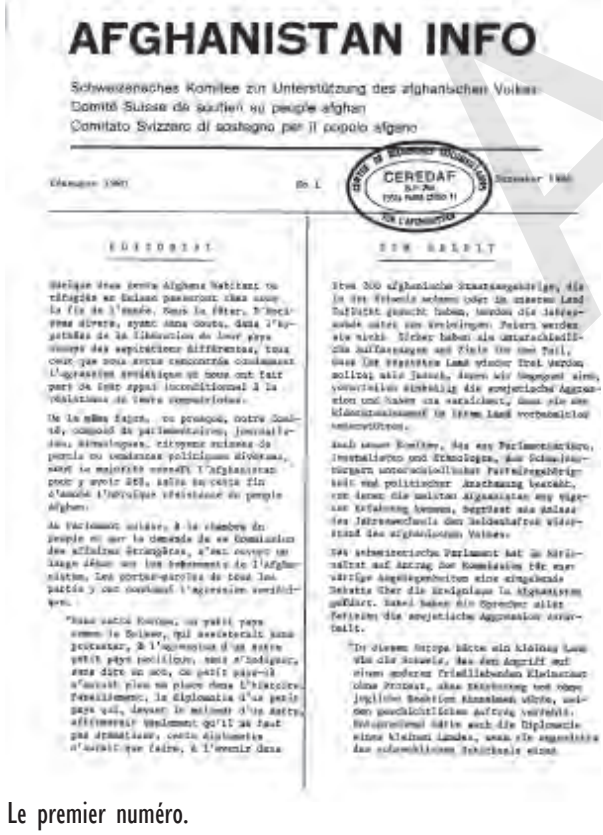
le Pakistan et bien d'autres d e s t i n a tions dans le monde. Il porte le numéro 80 et clôt une aventure éditoriale comptant près de 1920 pages, des centaines d'articles, des comptes rendus de très nombreux ouvrages et des illustrations provenant

* Ethnologue. Auteur avec Pierre Centlivres de nombreux ouvrages et articles sur l'Afghanistan. Rédacteur en chef d'Afghanistan Info de 1980 à 2017. pour beaucoup du fonds photographique de Pierre et Micheline Centlivres. Chaque numéro de 24 pages, au printemps et en automne, comportait une chronologie détaillée des événements survenus en Afghanistan depuis la parution du numéro précédent. Un réseau international d'amis, de collègues et de spécialistes possédant une connaissance exceptionnelle de l'Afghanistan a contribué en français, en anglais ou en allemand à enrichir son contenu. La consigne était simple : ne pas excéder deux à trois pages, aller à l'essentiel quel que soit le sujet. Des premiers numéros dactylographiés sur une vénérable machine à écrire, je suis passée dès 1985 à l'ordinateur qui a simplifié le travail d'écriture et la gestion du fichier d'adresses.

\section{Né après \\ l'intervention soviétique}

Afghanistan Info est né à l'initiative de quelques membres du Comité suisse de soutien au peuple afghan, une association créée au lendemain de l'intervention soviétique en 1979. Sans appui ni financement officiel, la parution du « bulletin », comme je l’appelle, a été rendue possible par le soutien de donateurs fidèles et généreux, sensibles aux malheurs soudains de l'Afghanistan, et sans doute au début préoccupés par l'avancée des troupes soviétiques vers l'Asie du Sud, puis par une crise sans fin. Les dons ont alimenté tout d'abord 
des projets d'aide à la population afghane ; ils ont servi ensuite à la couverture des frais occasionnés par la publication. Afghanistan Info était envoyé aux parlementaires et à des diplomates suisses, à des journalistes, à des organisations internationales et à tous ceux qui, d'est en ouest, étaient soucieux du sort des Afghans et de l’Afghanistan. Il est resté une publication gratuite.

\section{Décrypter}

Les quatre-vingts numéros d'Afghanistan Info témoignent de près de 40 ans de l'histoire contemporaine de l'Afghanistan dont ils décrivent et analysent les phases successives : occupation soviétique, survie du régime communiste de Kaboul, activités de résistance des moudjahidines, puis, après le retrait soviétique et la chute du régime, luttes fratricides des mouvements islamiques de résistance pour le pouvoir. Le « bulletin » a informé sur la condition de millions de réfugiés afghans au Pakistan et en Iran, analysé le contexte et les causes de l'irruption des Tâlebân, leur chute, l'espoir né avec la fin de leur régime et l'intervention étrangère, pour rendre compte enfin des résultats ambigus de cette dernière et des péripéties apparemment sans fin de la crise afghane. Au cours des années, auteurs et thématiques évoluent ; aux journalistes spécialisés, aux experts en stratégie militaire sollicités au lendemain de l'intervention soviétique, aux récits des jeunes et courageux « transborders » occidentaux, aux historiens du passé afghan, succèdent ou se joignent des spécialistes en science politique, des anthropologues, des économistes et des responsables d'ONG humanitaires. Tous contribuent à décrypter une situation complexe avec lucidité et un pessimisme fondé, mais non dénué d'espérance devant la remarquable résilience de la population afghane et les succès, modestes mais avérés dans certains domaines, tels l'infrastructure et la scolarisation.

$\mathrm{Au}$ travers du conflit et des crises qui le jalonnent, Afghanistan Info a suivi les permanences et les changements, à la fois la transformation de la société afghane et celle de la perception que les pays de la région et les grandes puissances ont d'un pays et d'une situation parfois difficiles à comprendre. Mais ce ne sont pas seulement les péripéties du conflit et les événements politiques dont le « bulletin » a voulu parler ; d'autre sujets touchant à l'actualité de l'Afghanistan y ont trouvé place : la renaissance culturelle du pays après la destruction des bouddhas de Bâmyân, en particulier les découvertes de sites nouveaux comme celui bouddhiste de Mes Aïnak et les trouvailles de Balkh, ou la contribution d'ONG françaises dans le suivi de la création de Conseils de villages ou dans l'effort pédagogique pour l'éducation des jeunes Afghans. On peut y lire aussi des récits portant sur la situation d'avant-guerre, à la fois lucides et nostalgiques, dans un temps apparemment

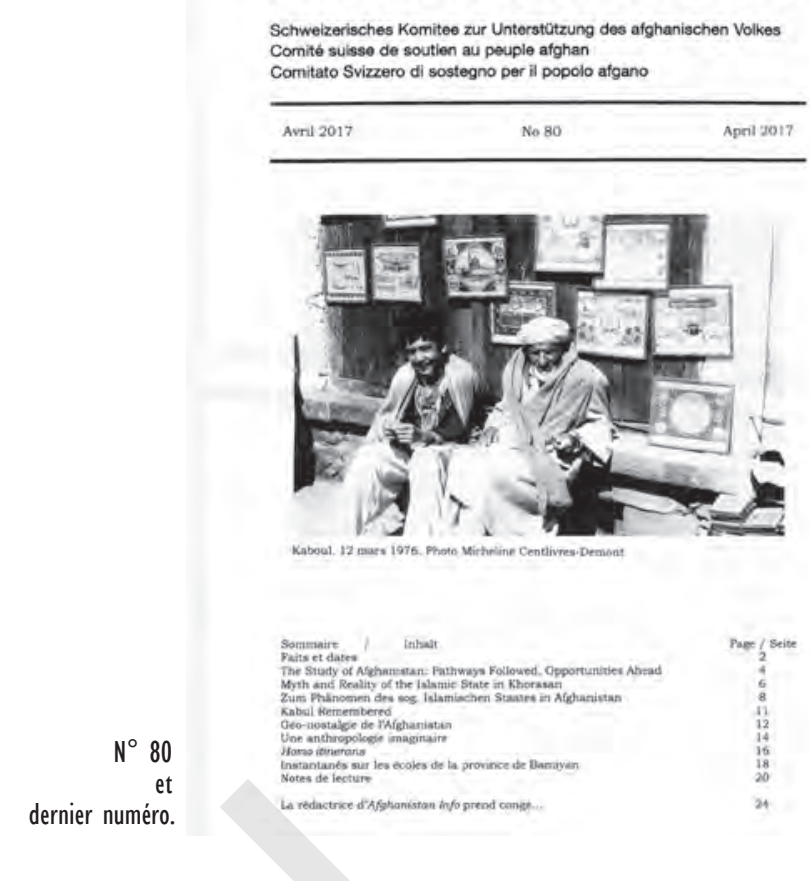

paisible, mais où l'observateur attentif pouvait déceler des fractures.

Avec la chute des Tâlebân, les ouvrages consacrés à l’Afghanistan ont été de plus en plus nombreux à parvenir au « bulletin "; des récits de guerre aux bandes dessinées, des romans aux témoignages, des ouvrages de science politique aux reportages et livres de souvenirs. Parmi eux les auteurs afghans occupent une place de plus en plus notable. Pierre et moi les avons lus avec une attention critique afin d'en faire l'objet d'un compte-rendu. Et, pourquoi ne pas le dire, la tâche de rédiger Afghanistan Info m'a valu une importante correspondance, des relations et des amitiés allant bien audelà des frontières de l'Europe. J'espère que ces liens ne disparaîtront pas avec la fin du Bulletin.

Alors qu'Afghanistan Info paraissait depuis près de 35 années, il m’a semblé utile de rassembler en un volume un « best of » réunissant certains des articles les plus pertinents, permettant de comprendre et l'évolution de la situation afghane et celle des regards portés sur elle. En février 2015 a paru à Londres un ouvrage ${ }^{1}$ comprenant 72 articles sélectionnés depuis les premiers numéros d'Afghanistan Info. Les articles qui avaient paru en français et en allemand y sont traduits en anglais.

Afghanistan Info achève sa carrière. Les Nouvelles d'Afghanistan heureusement continuent leur tâche d'information et d'analyse. La fin du « bulletin » ne signifie pas la fin de notre intérêt pour l'Afghanistan. Par le cœur et l'esprit, nous restons proches de ses habitants et de ses exilés partout dans le monde.

Mai 2017

1- Edited by Micheline Centlivres-Demont : Afghanistan. Identity, Society and Politics since 1980. London, I.B. Tauris, 2015, 317 p. ISBN 978-178453-081-5 


\section{Dernières nouvelles}

\section{Chronologie}

\section{Mars 2017}

- Le 1 : A Kaboul, deux attentats, revendiqués par les Tâlebân, font 21 morts et 104 blessés.

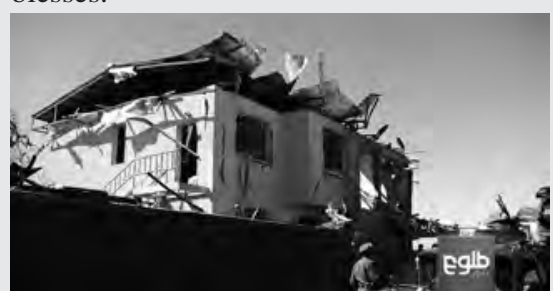

Kaboul, 21 morts, 104 blessés. Photo TN

- Le 2 : Achraf Ghani approuve la mention des mots «Afghan» et «tribu» sur les futures cartes d'identité informatisées.

- Le 3 : Une cérémonie a lieu à Machhad, en Iran, en hommage à deux hauts combattants afghans tués en Syrie.

- Le 4 : Daech décapite deux jeunes dans le district d'Atchin (Nangarhâr) puis, le 5, publie des photos de l'exécution.

- Le $5: 5$ soldats pakistanais et 10 insurgés sont tués dans des attaques terroristes menées depuis l'Afghanistan.

L'USAID lance un programme sur cing ans, d'un montant de 70 millions de dollars, pour améliorer le niveau de lecture en premier cycle primaire.

- Le 6 : Ce jour et les jours suivants, les forces pakistanaises tirent des centaines de roquettes sur la province du Kounar.

- Le 7 : Le Pakistan rouvre ses frontières pour deux jours, après 18 jours de fermeture.

Une délégation de cinq Tâlebân dirigée par Cher Abbas Stanikzai s’est récemment rendue en Chine.

- Le 8 : Des assaillants déguisés en médecins attaquent l'hôpital militaire de Kaboul, tuant 49 personnes et en blessant 63 . Daech revendique l'attentat. Les autorités suspectent en revanche les Tâlebân, qui s'en sont défendus, ou le réseau Haqqani.

- Le 9 : Accusée d'adultère une femme est lapidée après décision d'un tribunal tâleb dans le Badakhchân.

La Chine octroie une aide de 100 millions de dollars destinée aux réfugiés et aux rapatriés afghans.

- Le 11 : L'ancien chef des services de renseignement, Amrullah Saleh, est nommé ministre pour les réformes du secteur de la sécurité. Blessé dans un attentat il avait dû quitter ses fonctions il y a six ans.
Six policiers de Baghlân rejoignent les rangs des Tâlebân. Des insurgés de Daech décapitent 3 civils dans le district d'Atchin (Nangarhâr).

La Russie admet avoir avec les Tâlebân des «contacts limités» visant à assurer la sécurité des citoyens russes en Afghanistan et à créer des conditions favorables à des pourparlers directs entre le gouvernement afghan et les Tâlebân.

- Le 13 : Dans le district d’Obé (Hérat), des Tâlebân coupent une main et un pied d'un jeune homme accusé de vol.

Le ministère de la Justice annule les licences de plusieurs partis politiques pour «éviter le factionnalisme dans le pays». 60 partis politiques et 2593 groupes de la société civile ont reçu des licences. Il y avait précédemment 110 partis politiques.

Un pont de $120 \mathrm{~m}$ de long est inauguré dans le district de Matun (Khost).

32 civils et quatre policiers sont libérés d'une prison tenue par les Tâlebân dans le district de Nad-e Ali (Helmand).

Vingt-cinq personnes sont arrêtées dans la province du Pandjchir, dans le cadre d'une saisie de $700 \mathrm{~kg}$ de drogue.

- Le 15 : Des sénateurs américains proposent l'octroi de 2500 visas supplémentaires aux Afghans ayant travaillé pour les forces américaines.

L'Australienne enlevée en novembre 2016 est libérée.

24 personnes dont des généraux sont arrêtées suite à l'attaque de l'hôpital militaire à Kaboul le 8 mars. Le ministère de la Défense estime que «l'attaque a été planifiée à l'extérieur du pays».

Les autorités iraniennes remettent 200 prisonniers afghans au poste frontalier d'Islam Qala (Hérat). Ces prisonniers avaient été condamnés à mort ou à une peine de longue durée pour leur implication dans la contrebande de drogues en Iran.

Saluant la position de la Russie par rapport à ses contacts avec les Tâlebân, le ministère afghan des Affaires étrangères déclare que des efforts sincères des pays de la région peuvent s'avérer efficaces pour apporter la paix et la stabilité.

- Le 16 : L'Allemagne va injecter 20 millions d'euros dans le programme d'emploi qui sera mis en place dans les villages.

Le ministre afghan de l'Urbanisme et du Logement et l'ambassadeur de Chine signent un contrat pour la construction d'une première phase de 1400 appartements à Kaboul. Le gouvernement chinois s'est engagé à fournir 300 millions de dollars pour l'ensemble du projet couvrant 10000 appartements.

- Le 17 : Des insurgés de Daech auraient décapité trois représentants hazaras influents dans le district de Sayyad (Sar-e Pol).

Le conseiller à la sécurité nationale, Hanif Atmar, se rend à Moscou où il qualifie Moscou d'allié régional clé.

- Le 18 : Le Conseil de sécurité des Nations unies proroge d'un an le mandat de sa Mission d'assistance en Afghanistan.

- Le 19 : Un responsable d'Al-Qaïda, le Pakistanais Qari Yasin, auquel a été attribué l'attentat contre l'hôtel Marriott d'Islamabad le 20 septembre 2008, est tué par un drone américain dans la province de Paktika.

- Le 20 : Le Premier ministre pakistanais ordonne la réouverture de deux postesfrontières avec l'Afghanistan.

Lors d'une conférence au Louvre sept pays, parmi lesquels la France, l'Arabie saoudite et les Émirats arabes unis, et un donateur privé promettent 75,5 millions de dollars pour la sauvegarde du patrimoine culturel menacé par la guerre et le terrorisme. Les villes concernées sont prioritairement Palmyre, Bâmyân, Tombouctou et Mossoul.

- Le 22 : 9 policiers sont tués par un collègue sur la route Koundouz-Aliabad.

- Le 24 : La semaine dernière, 7 dirigeants tâlebân afghans ont eu des entretiens avec des responsables pakistanais. Islamabad tenterait d'encourager le groupe à participer à la conférence sur l'Afghanistan prévue à Moscou le 14 avril.

- Le 25 : Dans la province du Paktika, treize écoles qui étaient fermées pour raison d'insécurité ont rouvert.

- Le 26 : Quatre employés du ministère du Développement urbain et du Logement, accusés de détournement de 14 millions d'euros, sont condamnés à des peines de 7 à 20 ans de prison et à une amende de 27 millions d'euros.

- Le 27 : Le président du Conseil provincial de Hérat est condamné à deux ans et demi de prison pour corruption.

Achraf Ghani nomme Mme Narges Nehan au poste de ministre des Mines et du Pétrole.

Le Pakistan annonce avoir commencé la construction d'une barrière le long de sa frontière avec l'Afghanistan dans les zones tribales septentrionales de Mohmand et Bajaur afin d'entraver le déplacement des insurgés, initiative vivement critiquée par 


\section{Chronologie}

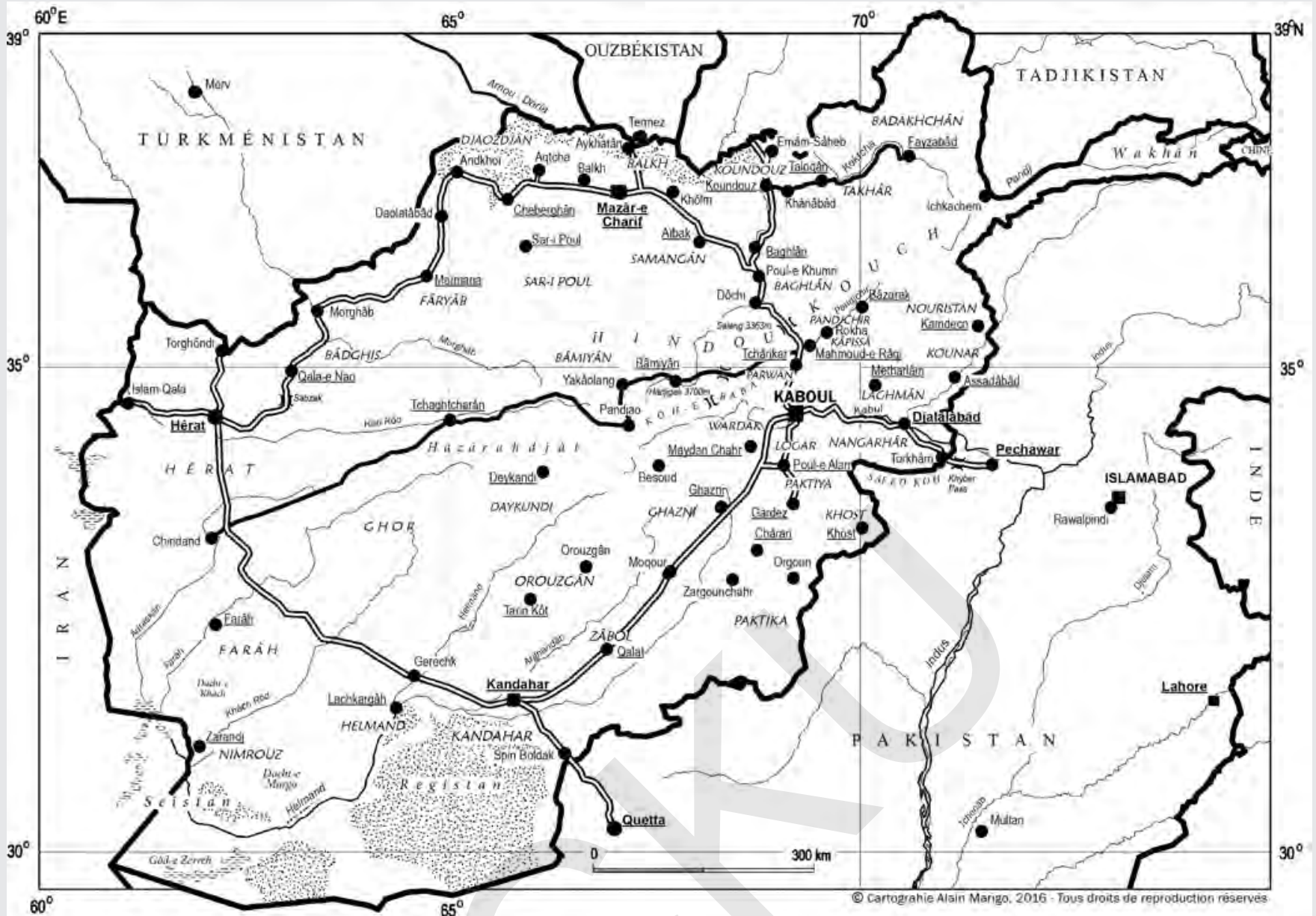

Kaboul. L’an passé, le Pakistan a achevé de creuser une tranchée de 1100 km le long de la partie méridionale de la frontière.

Les hauts responsables afghans de la sécurité obtiennent un vote de confiance après avoir été critiqués par les députés. Un Tâleb ayant rejoint le processus de paix dans le Nangarhâr avoue avoir reçu des instructions pour détruire le barrage de Darunta.

- Le 29 : Un général américain estime que la Russie fournit peut-être des armes aux Tâlebân afghans. Selon lui, «Tout comme l'Union soviétique, ils considèrent que cette partie du monde relève de leur sphère ».

\section{Avril}

- Le 1 : Latif Pedram, parlementaire et directeur général du Parti national du Congrès d'Afghanistan, déclare que son parti reconnaît la ligne Durand. Le 3, des parlementaires demandent son expulsion du Parlement.

- Le 2 : 30 tonnes d'explosifs sont saisies dans le district de Bagram (Parwân).

L'envoyé spécial de la Russie pour l'Afghanistan déclare "justifié » que les Tâlebân s’opposent à la présence militaire étrangère. Le président du Sénat demande aux États-Unis et à l'OTAN d'empêcher la Russie d'interférer en Afghanistan.

- Le 3 : Le Pakistan recommence à expulser des réfugiés afghans. Le programme avait été interrompu en décembre. Le HCR a réduit l'aide financière aux familles en partance, qui passe de 400 à 200 dollars par personne.

- Le 4 : L’Afghanistan et l'Australie signent un accord de développement d'une valeur de 320 millions de dollars concernant le secteur minier, l'agriculture et l'investissement d'entreprises australiennes. Dans les districts de Chahdjoï et de Chalamzai (Zâbol), 31 insurgés, dont 8 Pakistanais, sont tués.

- Le 5 : Un ancien chef de la police du district de Keran o Mundjân (Badakhchân) rejoint les Tâlebân.

Une délégation russe s'est rendue dans des centres de formation des Tâlebân dans le Nord-Waziristan au Pakistan.

- Le 6 : Le Pakistan a accordé 3000 bourses à des étudiants afghans.

Trois cent hectares de culture du pavot ont été éradiqués dans le Nangarhâr.

- Le 7 : Deux hauts dirigeants du réseau terroriste Haqqani sont tués dans le district de Geru (Ghazni).

Achraf Ghani achève une tournée d'une semaine en Australie, Indonésie et Singapour où plusieurs accords ont été signés. Selon le gouverneur du district de Raghistan (Badakhchân) les Tâlebân engrangent 40000 euros par mois avec l'exploitation de la mine d'or du district.

- Le 8 : Un soldat américain est tué dans la même province.

- Le 11 : Des sénateurs demandent une enquête sur la distribution par le gouverneur de la province de Koundouz d'aides à une soixantaine de familles de combattants tâlebân tués.

200 soldats américains sont déployés dans l'Helmand.

- Le 12 : L’Assemblée Nationale suspend quatre députés, dont deux femmes, pour leurs absences. Cinq autres seront suspendus le 22.

Un attentat suicide à Kaboul près du ministère de la Défense, revendiqué par Daech, fait 5 morts.

- Le 13 : La première pierre d'une école pour 60 écoliers sikhs et hindous est posée à Djalâlâbâd avec l'aide financière du Swedish Committee.

Les Etats-Unis larguent une bombe de 9,8 tonnes, la plus grosse de leur arsenal, contre Daech sur un réseau de tunnels et de grottes dans le district d'Atchin (Nangarhâr). Elle tue au moins 94 combattants 
La situation des réfugiés, internes ou provenant des pays limitrophes, constitue un élément important de préoccupation tant les risques de déstabilisation économique et sociale sont importants. Ces retours sont en lien avec la crise afghano-pakistanaise qui affecte tant les échanges entre les deux pays que les prix en Afghanistan. A noter (voir Secteur Agri-Agro) la mention de fortes précipitations cet hiver qui devraient bénéficier au secteur agricole et avoir un impact positif sur la population. R. L.

\section{Réfugiés}

Aide d'urgence: I'ONU réclame 550 millions de dollars :

Selon les données officielles des Nations unies, plus de 623000 Afghans ont été chassés de chez eux en 2016 pour trouver refuge ailleurs dans le pays, «le chiffre les plus élevé jamais enregistré», en hausse de $30 \%$ sur un an. Pour 2017, l'ONU s'attend à 450000 nouveaux déplacements dus à l'insécurité ou aux désastres naturels et à un million de retours supplémentaires des pays voisins. Selon l'ONU, 1,6 million d'Afghans souffrent de malnutrition et un million d'enfants de malnutrition sévère. (AFP 21/01)

- Le Fonds monétaire international (FMI) met en garde contre les risques d'aggravation de la situation économique et sociale en Afghanistan avec le retour massif de réfugiés dans le pays. II appelle la communauté internationale à fournir rapidement de l'aide à Kaboul. Selon un rapport du FMI, 2,5 millions réfugiés de plus devraient arriver dans les 18 mois. Cela entraînerait une augmentation rapide de $10 \%$ de la population afghane. Ces réfugiés sont confrontés à l'insécurité et à la pauvreté et leur présence augmente la pression sur les services d'éducation et de santé, tout en alimentant une hausse des loyers et des prix. (RFI 28/01)

- Au cours de la dernière année afghane (20 mars 2016-20 mars 2017), 1,1 million de réfugiés afghans sont retournés dans leur pays en provenance d'Iran et du Pakistan, selon le ministère des Réfugiés. (Tolo 20/03)

\section{Commerce extérieur}

- Le 31 janvier, le ministère afghan du Commerce et des Industries a déclaré que le commerce avec l'Iran a considérablement augmenté. Les matériaux de construction, les matières premières et les denrées alimentaires sont les principales marchandises importées d'Iran. Mais de grandes quantités de sel, de carburant et de gaz sont aussi importées illégalement, représentant près d'un milliard de dollars par an. (Tolo News 31/01)

- Le 13 mars, la Chambre de commerce et d'industrie afghane a annoncé que la contrebande de marchandises pakistanaises vers l'Afghanistan a triplé depuis la fermeture des frontières entre les deux pays. Plus de 50.000 camions chargés de marchandises pour l'Afghanistan sont bloqués au Pakistan. Aussi les marchandises pakistanaises entrent illégalement sur les marchés d'Afghanistan sans droits de douane et sont vendues jusqu'à quatre fois plus chers que la normale. (Tolo News 13/03)

Selon cette même chambre (le 18 mars), le Pakistan a perdu 3 milliards de dollars en exportations en fermant sa frontière depuis plus d'un mois. Les produits iraniens, turcs et indiens remplacent les produits pakistanais sur les marchés. Les prix ont fortement augmenté, mais cela a également entraîné un boom dans la vente de produits nationaux.

- Le 9 avril, la Chambre du Commerce et de l'Industries d'Afghanistan a déclaré que l'Iran a conservé sa position de partenaire commercial le plus important du pays en 2016.

Les échanges commerciaux entre les deux pays en 2016 étaient de 1,8 milliard de dollars. Alors que, pour la même année, ceux entre l'Afghanistan et le Pakistan sont de 1,3 milliard de dollars, 1 milliard de dollars avec la Chine, 700 millions avec le Turkménistan et 500 millions avec le Kazakhstan. (Tolo News 09/04)

Souces principales : le Bulletin du CEREDAF

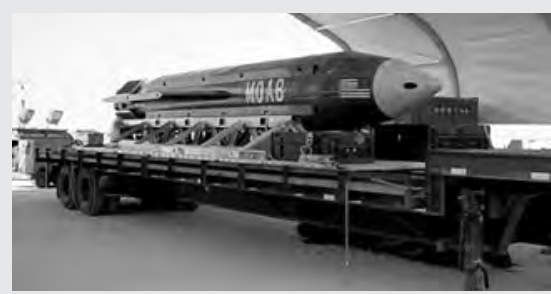

MOAB, la plus grosse bombe américaine. Photo DR

et en blesse 90, selon le gouvernement afghan. Il n’y aurait aucune toute victime civile.

- Le 14 : La Russie réunit une conférence internationale sur l'Afghanistan. Sont invités l'Afghanistan, les pays d'Asie centrale, la Chine, l'Inde, le Pakistan et l'Iran. Les Etats-Unis invités, n’ont pas répondu. Aucun ministre afghan n’est présent.
- Le 15 : Hâmed Karzaï déclare que les États-Unis ont violé la souveraineté de l'Afghanistan et insulté l'honneur des Afghans en larguant leur bombe massive. Il déclare aussi que le Pakistan avait exigé la reconnaissance par l'Afghanistan de la Ligne Durand comme frontière internationale entre les deux pays pendant sa présidence.

- Le 16 : Dans le district de Sayad (Sar-e Pol), des Tâlebân exécutent un enfant et trois femmes soupçonnés de coopération avec le gouvernement.

Le ministère de la Défense lance une opération, l'«Opération Khalid», dans les zones à forte menace terroriste (Sar-e Pol, Faryâb, Baghlân, Koundouz, Helmand, Orouzgân, Badghis et Farâh).

- Le 17 : Deux tonnes d'explosifs sont sai- sies à Kaboul.

Achraf Ghani démet de ses fonctions Ahmad Zia Massoud, représentant spécial pour les réformes et la bonne gouvernance, pour ses critiques envers le gouvernement. Un général afghan est tué par les Tâlebân dans le district de Khânâbâd (Koundouz). - Le 18 : Le ministère des Finances annonce une politique de budgétisation provinciale planifiée par les autorités locales en consultation avec les populations. Quatre ministères (Agriculture, Santé, Développement rural et Education) ont commencé à la mettre en œuvre.

Selon des habitants du district de Puchrod (Farâh), les Tâlebân ont commencé à collecter des taxes auprès des propriétaires fonciers, ce que les Tâlebân réfutent.

- Le 19 : Hâmed Karzaï déclare à Voice of America que Daech est un «outil» des États-Unis. Je ne fais pas de différence entre Daech et les Etats-Unis, dit-il, ajoutant : " Les Tâlebân sont une réalité majeure en Afghanistan aujourd'hui. Lorsqu'une force contrôle $50 \%$, les pays n'ont d'autre choix que de leur parler. Les États-Unis eux-mêmes leur parlent quand ils les rencontrent dans leur bureau du Oatar. Alors pourquoi la Russie n'aurait pas le droit de leur parler aussi ?».

Selon les autorités, 13 dirigeants de Daech auraient été tués par la bombe américaine dans le Nangarhâr. Parmi les insurgés tués, il y aurait des ressortissants indiens, pakistanais, ouzbeks, russes, indiens, philippins, bangladechis et tadjiks.

- Le 20 : Les retours de réfugiés afghans du Pakistan s'accélèrent depuis début avril, atteignant le rythme d'un millier par jour, sans aucun soutien pour se réinstaller, selon le Conseil norvégien des réfugiés.

Farouq Wardak est nommé ministre d’État aux Affaires parlementaires.

- Le 21 : 144 soldats sont tués et 65 blessés dans l'attaque de leur base par une dizaine de Tâlebân, près de de Mazar-e Charif. L'attaque a visé des hommes pendant la prière et d'autres au réfectoire. Les Tâlebân revendiquent l'opération.

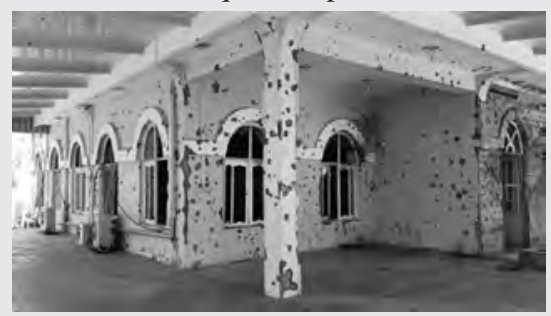

Mazar-e Charif, impacts sur un bâtiment de la base militaire. Photo DR

- Le 22 : L’Assemblée Nationale suspend cinq autres parlementaires pour leur absence durant 20 sessions d'affilée.

- Le 24 : Des insurgés attaquent une base américaine près de Khost. Quatre soldats des Forces spéciales afghanes sont tués. Selon le chef du Pentagone lors d'une visite surprise en Afghanistan, 2017 sera une 
année difficile pour les forces afghanes. Le leader du Hezb-e Islami, Gulbudin Hekmatyar, arrive à Djalâlâbâd,

Le ministre de la Défense et le chef d’État-major présentent leur démission après l'attaque sur la base de Mazar.

Le recours à la torture augmente en Afghanistan dans le cadre de la lutte contre les insurgés et vise aussi les détenus mineurs, indique un rapport de l'ONU. Parmi les 469 détenus interrogés par les enquêteurs, $39 \%$ ont fourni des récits crédibles et fiables de tortures ou autres formes de traitements inhumains et dégradants pendant leur détention.
Reçu à l'ambassade de Chine à Kaboul, Hâmed Karzaï a déclaré que les pays de la région ne doivent pas laisser les États-Unis détruire le pays en le transformant en terrain de rivalité. Le lendemain il se rend à Moscou.

- Le 26 : Une route asphaltée de $33 \mathrm{~km}$ reliant le district de Djuwayn à Farâh est mise en service.

Suite à l'attaque de la base militaire de Mazar-e Charif, 135 militaires servant sur la base sont interpellés.

- Le 27 : Abdul Hasib, considéré par le Pentagone comme le chef de l'Etat islamique en Afghanistan et le principal res- ponsable de l'attaque de l'hôpital militaire de Kaboul, a probablement été tué dans le Nangarhâr, au cours d'une attaque où deux militaires américains ont perdu la vie. L'information sera confirmée en mai. Plusieurs autres hauts responsables de Daech et 35 combattants ont été tués.

- Le 28 : A Mehterlâm, le gouverneur du Laghmân reçoit G. Hekmatyar qui appelle les Tâlebân à cesser le combat. Il met en garde les «forces étrangères qui ne peuvent gagner cette guerre». G. Hekmatyar critique aussi ces «médias qui diffusent de la musique, des danses et des séries alors que chaque jour des centaines d'Afghans meu-

\section{Hommages}

\section{Marjan KAMAL}

Marjan est née à Kaboul le 21 novembre 1976. Elle avait juste 3 mois quand ses parents, arrivés en France, s'installent à $\mathrm{Pa}$ ris, où Marjan fait toute sa scolarité et ses études supérieures. .

En 1999 elle obtient une Maitrise en Histoire du Moyen-âge à la Sorbonne et, en parallèle, une licence en Islamologie et en langue pachtou aux Langues Orientales auprès du professeur Maryam Abou-Zahab.

C'est à ce moment que j'ai fait connaissance avec la jeune Marjan. Son physique, son esprit vif, son intelligence m'ont séduite. Etant présidente du Centre de Recherches et d'Etudes documentaires sur l'Afghanistan (CEREDAF), je lui proposais

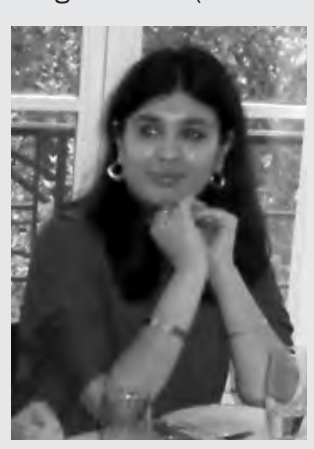
de nous aider à améliorer notre fonds documentaire et d'assurer les permanences de notre association. Elle a accepté avec enthousiasme.

En 2004 elle obtient son diplôme de DEA en Analyse Comparative des Aires Politiques - section monde musulman - à Science Po Paris sous la direction de Gilles Kepel. Son mémoire a porté sur la reconstruction de l'Etat afghan dans le contexte post guerre.

A partir de 2005 elle a entrepris des travaux de recherche pour une thèse de doctorat sur les dynamiques du conflit armé en Afghanistan qui l'ont amené à entreprendre des travaux sur le terrain et à sillonner son pays natal plusieurs années durant et à rencontrer tous les principaux protagonistes du conflit afghan. Avec son professeur de Langues orientales, elle effectue ainsi des voyages en Afghanistan et au Pakistan dans les zones tribales, où elle a l'occasion notamment de visiter des sanctuaires et des tombes de saints.

En parallèle, pour financer ses travaux de recherche, elle participe au conseil en développement(de quoi s'agit-il ?) sur différentes questions comme la reconstruction de l'Etat, le travail des ONG et l'action des forces de l'OTAN.

Infatigable jusqu'au bout de ses forces, une semaine avant de nous quitter, Marjan faisait encore des projets d'écrire et de mettre sur papier toutes ses expériences vécues.

Elle nous a quittés au moment de la plus grande fécondité de son esprit.

Le CEREDAF lui doit beaucoup, malgré la charge de ses études. Durant ces dernières années, Marjan était membre de son Conseil d'administration et collaborait à la rédaction du Bulletin du CEREDAF. Elle a également publié plusieurs articles dans Les Nouvelles d'Afghanistan où elle a essayé de décrypter pour nous l'actualité afghane.

Marjan était perfectionniste, exigeante, passionnée, altruiste. Elle a toujours voulu aller jusqu'au bout de sa recherche, le temps ne comptait pas pour elle. Elle nous a quittés dans la fleur de son âge.

Nous présentons nos condoléances et toute notre affection à sa famille.

Merci Marjan pour ce que tu as fait et que tu nous la laissé.

\section{Véra MARIGO}

\section{Mohammad FAZEL - SAHEBZADA}

Ancien Vice-ministre de l'Education $\mathrm{Na}$ tionale, ancien doyen et professeur à l'Université de Kaboul à l'époque du roi, Ministre de l'Education Nationale à l'époque des modjahedin et résistant actif contre le communisme et l'invasion soviétique, M. Fazel était aussi écrivain et traducteur de plu- sieurs livres et documents pédagogiques et éducatifs.

II appartenait à une grande famille, résistante et intellectuelle, habitant aux environs de Tcharikar à $60 \mathrm{~km}$ au nord de Kaboul. Son arrière-grand-père, Mir Masdjid Khan Sâhebzâda, un des célèbres résistants de la deuxième guerre anglo-afghane et son père, Mir Mohammad Sidiq Khan, général durant la 3ème guerre anglo-afghane, ont assuré l'indépendance de l'Afghanistan contre les envahisseurs.

Pohand Fazel, né en 1922 à Dehqâzi, près de Tcharikar, a fait ses études supérieures à la faculté de lettres de Kaboul. En 1965, il a fait un Master en psychologie éducative à l'université de Columbia aux Etats-Unis. II est décédé le 3 mars 2017 à Cergy-Pontoise et a été enterré en Afghanis$\tan$

M. Fazel a consacré toute sa vie à l'éducation des enfants afghans, à l'amélioration des conditions d'études pour les filles et les garçons. II s'est aussi invest dans la construction d'écoles dans toutes les régions d'Afghanistan sans exception. Il pensait que la seule possibilité de sorti son pays de la misère était qu'il prenne son destin en main, et que cela n'était possible que par l'éducation des jeunes.

Nous assurons sa famille de notre sympathie

\section{Anne CHAIGNON}

Adhérente d'AFRANE de la première heure, Anne Chaignon, sœur aînée de Vincent Schneiter, vient de décéder le 23 mars dernier à l'âge de 77 ans. Généreuse, fidèle en amitiés, elle a soutenu AFRANE matériellement en donnant des objets d'artisanat. Elle a aussi aidé avec son mari des réfugiés afghans.

Nous présentons nos condoléances à Vincent et à toute sa famille. 


\section{Corruption}

- Le 18 mars, l'Union européenne a exhorté le gouvernement afghan à prendre des mesures immédiates pour mettre fin à l'exploitation minière illégale dans le pays.

L'UE a suggéré au gouvernement afghan de nommer un ministre des Mines et du Pétrole capable et digne de confiance pour revitaliser et normaliser le secteur minier. Les entreprises qui ne paient pas leurs impôts volontairement devraient voir leurs licences révoquées. L'UE a demandé de réexaminer toutes les licences minières qui ne produisent pas de revenus pour le gouvernement afghan. (Pajhwok Afghan News 19/03)

- L'étude du Réseau afghan de lutte contre la corruption intitulée «Pillage des mines et scandale apparent au ministère des Mines" a dévoilé que de hauts fonctionnaires du ministère des Mines auraient attribué 217 contrats d'extraction en échange de 10 millions de dollars en pot-de-vin. (Pajhwok Afghan News 11/03)

\section{Secteur agri-agro}

- Le 6 février, l'Organisation centrale des statistiques a déclaré que les récentes fortes chutes de neige de cette année favoriseront l'agriculture. Une grande partie du développement économique de l'Afghanistan dépend de la croissance du secteur agricole qui représente $80 \%$ de l'activité dans le pays. Les agriculteurs afghans sont optimistes quant aux récoltes de cette année, les pluies et les chutes de neige ont mis fin aux pénuries d'eau dans de grandes parties du pays. (Tolo News 06/02)

Selon le ministère de l'Agriculture et de l'Irrigation, plus de 700 millions de dollars ont été investis dans des élevages de volailles et la production de graines dans le pays au cours des dernières années.

Récemment, le gouvernement a instauré un système tarifaire sur les poulets et les œufs importés dans le but de promouvoir la production locale. Cependant ces entreprises ne peuvent satisfaire que $49 \%$ des besoins en volailles du pays et 25\% des besoins en œufs. (Tolo News et Khaama Press 23/01)

\section{Divers}

- Le 8 janvier, une entreprise chinoise de construction a signé un contrat pour construire l'axe routier Dare-e-Sof (Samangân) à Yakaolang (Bâmiyân) sur 178km dans le centre de l'Afghanistan pour une valeur de 205 millions de dollars. Le premier tronçon de cet axe, qui relie Mazar-e Charif à Yakaolang, est déjà terminé. Le ministère est en train de planifier la 3ème phase, une route de $550 \mathrm{~km}$ qui reliera Bâmiyân à la province de Kandahar. (Xinhua 09/01)

- Le 27 avril, la Chambre de Commerce et de l'Industrie d'Afghanistan a déclaré que l'absence de travailleurs qualifiés dans le pays a un effet négatif sur les entreprises et affecte les investissements dans le pays. Près de $80 \%$ des travailleurs qualifiés sont des étrangers.

Le Centre de formation professionnelle afghano-coréen assure la formation à environ 1000 ouvriers dans différents domaines tels que les réparations automobiles, la maintenance générale, l'électrotechnique, et la construction. Le ministère a déclaré que 46 autres centres de formation professionnelle seront créés dans un proche avenir. (Tolo News 28/04)

Souces principales : le Bulletin du CEREDAF

rent noyés dans leur sang».

Maolawi Daoud, ancien gouverneur tâleb de Kaboul, et son chauffeur sont abattus près de Peshawar, au Pakistan.

Une délégation de haut niveau de l'armée pakistanaise se rend en Afghanistan pour des entretiens au sujet de mesures de coordination des frontières. Elle offre un traitement gratuit aux Afghans blessés le 14 avril à Mazar-e Charif.

- Le 29 : La Banque asiatique de déve- loppement fournira 300 millions de dollars pour des projets d'infrastructure en Afghanistan (routes, parc solaire dans la province du Nangarhâr...).

- Le 30 : Compte tenu de la détérioration de la situation, l'OTAN étudie une augmentation des effectifs de sa mission «Resolute Support», qui compte actuellement 13000 hommes.

Le deuxième vice-président Sarwar Danech se rend dans la province du Nangarhâr

\section{La situation des Afghans rapatriés}

Amnesty International a attiré l'attention, le 2 mai, sur la situation des Afghans rapatriés dans leur pays. Voici la substance de ce communiqué.

Les déboutés afghans du droit d'asile au sein de l'UE sont renvoyés par différents Etats-membres, dont l'Allemagne, dans leur pays alors même que celui-ci est confronté à un nombre croissant de victimes civiles, dans des zones de plus en plus nombreuses, Ce renvoi intervient au titre de l'accord de réadmission, pour le moins surprenant et dans son contenu et dans sa forme, conclu en octobre entre I'UE et le gouvernement afghan, Or, force est de constater que, jusqu'à présent, aucune ligne budgétaire afghane spécifique n'a vraiment été dédiée aux programmes d'aide et de développement des personnes déplacées et rapatriées, et le manque d'engagement et de compétences des institutions et acteurs afghans concernés conjugué à la corruption est patent.

L'Allemagne a suspendu ces rapatriements suite au terrible attentat du 30 mai. pour accueillir G. Hekmatyar.

\section{Mai}

- Le 1 : Achraf Ghani signe un décret pour la libération du premier contingent de 70 prisonniers du Hezb-e Islami.

- Le 2 : Des affrontements éclatent à la frontière entre le Pakistan et l'Afghanistan entre soldats des deux pays. L'armée pakistanaise fait état d'un mort et de 18 blessés et accuse l'armée afghane d'avoir ouvert le feu sur une équipe effectuant un recensement dans un village proche de la ville frontalière de Chaman.

- Le 3 : Le ministère de la Défense déclare avoir défini une stratégie de guerre sur quatre ans avec le soutien des Etats-Unis.

Le Hezb-e Islami reconnait que 13 prisonniers non encore libérés ont été impliqués dans la planification d'attentats suicides.

- Le 4 : Un attentat revendiqué par Daech frappe un convoi blindé de l'OTAN dans le centre de Kaboul, faisant 8 morts et 28 blessés, dont 3 soldats étrangers. Les victimes sont pour la plupart des civils.

Achraf Ghani accueille Gulbuddin Hekmatyar au Palais présidentiel. Son retour à bord d'un convoi de deux cents voitures puissamment armées, mitrailleuses en batterie, intervient sept mois après la signature de l'accord de paix. Depuis une semaine de grands portraits de Hekmatyar ont fait leur apparition, rapidement déchirés ou maculés.

Le Sénat des États-Unis approuve le financement de 2500 visas d'immigrant supplémentaires pour les interprètes afghans et le personnel qui ont aidé les troupes américaines.

Achraf Ghani s'entretient avec le chef du service d'intelligence militaire pakistanais. Il lui remet des documents concernant des attaques perpétrées en Afghanistan par des insurgés utilisant le sol pakistanais.

- Le 7 : L’armée pakistanaise déclare avoir détruit cinq postes afghans près de Chaman. Selon le gouvernement afghan, le recensement que réalisaient les Pakistanais se déroulait dans le village afghan de Loqman et non en territoire pakistanais.

- Le 9 : Une équipe afghano-pakistanaise enquêtera le long de la section contestée de la frontière à Chaman-Spin Boldak.

- Le 11 : Hekmatyar déclare que les combattants du Hezb-e Islami ne déposeront pas leurs armes, contrairement à l'accord passé avec le gouvernement. Son porte-parole estime «nécessaire» pour leurs 3500 combattants de garder leurs armes pour se protéger des Tâlebân et de Daech.

- Le 16 : Le Procureur général déclare avéré l'abus sexuel subi par Ahmad Ishchi impliquant le général Dostum.

- Le 17 : Selon le chef de la police de la province de Baghlân, des experts militaires russes, pakistanais, égyptiens et tchétchènes seraient arrivés dans la province 


\section{Principaux lieux d’affrontement}

Nous avons regroupé, province par province les événements ayant fait le plus de victimes, tels que recensés par le Bulletin du CEREDAF. Les trois quarts des provinces apparaissent dans cette liste. L'aviation américaine a largué plus de bombes sur l'Afghanistan en avril que pendant n'importe quel autre mois depuis 2012, et sept fois plus qu'en avril 2016 selon le Pentagone. La multiplication des raids aériens, essentiellement sur le Nangarhar, à l'est, s'explique en partie par la volonté de Washington d'empêcher l'implantation durable de Daech en Afghanistan.

Badakhchân : Le 28 avril le district de Zebak serait tombé aux mains des Tâlebân. 20 insurgés sont été tués dans des bombardements menés le 1er mai après la prise du district. Les Tâlebân tentent sans succès d'envahir le district voisin d'Echkachem pour avoir un accès direct au district pakistanais de Chitral. Le 10 mai, Les Tâlebân sont repoussés des districts de Zebak et d'Echkachem. Le même jour, les Tâlebân lancent une offensive sur le district de Raghistan. Le 28, selon des responsables locaux, plusieurs villages du district de Tagâb seraient tombés aux mains des Tâlebân.

Baghlân : Des Tâlebân s'emparent de Tala-o Barfak le 1er mars. L'armée reprend le district le 3. Le 19 avril, le gouverneur tâleb de la province et 25 insurgés sont tués dans un raid aérien près de Pol-e Khomri. Le 24 mai, l'axe routier Baghlân-Mazar est fermé à la circulation suite à des attaques des Tâlebân depuis la veille au soir.

Badghis : Le 27 mai, une attaque des Tâlebân fait 36 morts (22 insurgés, 6 soldats et 8 civils) et une cinquantaine de blessés parmi les Tâlebân et les civils.

Balkh : Le 21 avril 144 soldats sont tués et 65 blessés dans l'attaque de leur base par une dizaine de Tâlebân, près de Mazar-e Charif.

Djaozdjân : Le 26 avril 76 Tâlebân et 15 combattants de Daech sont tués et 56 Tâlebân son blessés lors d'affrontements entre eux dans le district de Darzâb.

Farâh : 8 civils tués et 22 blessés à Bala Boulouk par les forces afghanes le $3 / 3$.

Faryâb : Le 12, les Tâlebân occupent le district de Beltcherâgh.. Le 1er mai, les forces afghanes reprennent le district de Ghormach. Le 15 mai, une attaque des Tâlebân contre Maïmana, capitale de la province est repoussée par les forces afghanes.

Ghazni : Le 19 mai, des Tâlebân attaquent la ville de Ghazni et les districts de Waghaz et Deh Yak. Au cours des affrontements, 25 insurgés et 2 soldats sont tués.

Helmand : 20 insurgés tués à Khânnechin le 1/3 ; 27 le 11/3. Le 14 mars, 24 insurgés sont tués par des frappes afghanes et américaines. Après avoir dégagé Lachkargâh, les forces afghanes reprennent des parties du district de Nad-e Ali et rouvrent la route qui y mène, fermée depuis six mois. Le 23 mars, les Tâlebân prennent Sangin. Le 28, 66 insurgés sont tués dans des frappes aériennes. Le 9 mai, un important centre de commandement des Tâlebân est détruit dans le district de Nad-e Ali. Le 18, 23 insurgés sont tués lors de raids aériens américains dans le district de Garmser. Le 20, 33 insurgés sont tués dans le district de Nad-e Ali. Le 25, 25 agents de sécurité sont tués et de nombreux autres blessés par un kamikaz dans le district de Wachir. Le 26, 11 personnes sont libérées d'une prison tenue par les Tâlebân dans le district de Nad-e Ali. La dernière semaine de mai, 110 Tâlebân sont tués dans ce district.

Hérat : Le 21 et le 22 avril, huit Tâlebân sont tués lors de luttes intestines dans le district de Chindand.

Kandahar : 20 insurgés tués par l'OTAN à Nech le $8 / 3.16$ soldats sont tués dans le district de Khâkrez le 28/3. Le 4 avril, 30 insurgés sont tués lors de raids aériens dans le district de Chah Walikot. Le 19 mai, 22 insurgés et 8 policiers sont tués dans des attaques sur les districts de Khakrez, Arghestan et Nachin. Le 22, 11 soldats et 20 insurgés sont tués lors d'une attaque dans e district de Chah Wali Kot Le 25, 13 soldats sont tués et 8 blessés dans le district de Maïwand. Le $26: 18$ soldats sont tués, 16 blessés et 4 enlevés dans le district de Shah Walikot. Dans l'affrontement, 7 Tâlebân sont tués.

Khost : Le 27 mai, un attentat à la voiture piégée revendiqué par les Tâlebân et visant apparemment une milice financée par la CIA fait 13 morts et 8 blessés, au premier jour du ramadan. Plusieurs membres de la milice figurent parmi les victimes. Cette milice, qui serrait encadrée par la CIA, est accusée de tortures et détentions arbitraires.

Koundouz : Le 5 avril, le vice-gouverneur tâleb de la province est tué avec 13 insurgés lors d'un raid aérien. Le 17, 16 insurgés, dont le gouverneur tâleb de la province de Takhâr, sont tués à Dacht-e Artchi. Le 24, dans le district de Tchahardara, 35 insurgés sont tués et 6 arrêtés. Le 5 mai, la route Koundouz-Talogân est fermée suite à de violents affrontements dans l'est de la province. Le 6, les Tâlebân s'emparent du district de Qalae Zâl. Des centaines de civils s'enfuient. Le $7: 37$ insurgés, dont 5 commandants, sont tués dans ce district qui est repris le 16. De lourds combats ont lieu dans les alentours de Koundouz et se prolongent plusieurs jours. Le 13, 55 Tâlebân sont tués et 49 blessés dans l'opération lancée par les forces de sécurité pour libérer l'axe routier Koundouz-Khânâbâd. Le 25, cette route est à nouveau fermée suite à des attaques tâlebân contre des postes de sécurité.

Laghmân : 20 insurgés tués le 1/3. A la mi-mars 70 Tâlebân sont tués et 50 blessés en tentant de s'implanter dans la province. Le 17 mai, 45 insurgés sont tués, 20 blessés et 2 commandants arrêtés.

Logar : Au cours d'une opération de vingt jours en mars dans les districts de Mohammad Agha Barak-e-Barak et Tcharkh, 23 insurgés sont tués 28 blessés et 20 arrêtés.

Nangarhar : C'est dans cette province, plus précisément dans les districts d'Atchin, Tchaparhar que le nombre de victimes, notamment dans des bombardements visant Daech semble avoit été le plus élevé. De l'ordre d'un millier. Parmi les morts, Sayed Omar Bajawari, l'un des plus hauts responsables de Daech.. Le 13 avril, les EtatsUnis larguent une bombe de 9,8 tonnes, la plus grosse de leur arsenal, contre Daech sur un réseau de tunnels et de grottes du district d'Atchin Elle tue au moins 94 combattants et en blesse 90 , selon le gouvernement afghan. II n'y aurait aucune victime civile. Le 30 , lors d'un conflit entre Tâlebân et Daech dans le district de Tchaparhar, 21 Tâlebân et 7 combattants de Daech sont tués.

Orouzgân : Le 22 mars, 17 corps de soldats son trouvés dans une base assiégée par les Tâlebân depuis six mois. Le 2 avril, 54 Tâlebân sont tués et 32 blessés au cours d'une semaine d'opérations dans les environs de Tarinkot. Le 25 et le 26, 61 insurgés sont tués près de Tarinkot. Dans la nuit du 4 au 5 mai, 35 insurgés sont tués et 45 blessés dans le district de Dehrawoud. Le 14 113 Tâlebân sont tués et une zone stratégique de Tarinkot est libéréee des insurgés.

Paktika : Le 14 mars, 20 insurgés, dont 2 commandants du réseau Haqqani, sont tués dans le district de Zeluk. Le 20 : 33 Tâlebân sont tués par des drones américains.

Samangân : Le 9 mai, des insurgés tentent de prendre le contrôle des mines de charbon du district de Dara-e Souf Bâlâ

Takhâr : Du 2 au 4 mai, 11 agents de sécurité, 4 civils et 67 Tâlebân sont tués dans le district de Khwadja Bahauddin. Une vingtaine de villages sont libérés des Tâlebân..

Zâbol : Le 21 mai, une vingtaine de policiers sont tués et une quinzaine blessés au cours de six attaques coordonnées dans le district de Châhdjoy. Le 27, 6 policiers sont tués par un collègue dans le district de Chinkai. pour former et soutenir les Tâlebân. Les Tâlebân déclarent qu'un accord de paix avec le gouvernement afghan serait une reddition contraire à la foi islamique. - Le 18 : Selon Atta Mohamad Nour, gouverneur de la province de Balkh, les régions du Nord deviendront une deuxième Syrie si des mesures immédiates ne sont pas prises pour éliminer les menaces des groupes terroristes.

Selon le Bureau du chef de l'exécutif il n’y a pas de preuve démontrant un soutien militaire de la Russie aux Tâlebân.

- Le 19 : Les Turkmènes du nord de l'Afghanistan ont formé leur propre groupe armé pour lutter contre les Tâlebân, jugeant les forces afghanes incapables de protéger la région.

- Le 20 : Une Allemande est tuée à Kaboul et une Finlandaise de l’ONG suédoise " Operation Mercy » est enlevée.

Achraf Ghani se rend en Arabie saoudite, où il rejoint les dirigeants de vingt nations musulmanes pour un sommet islamique. Ce voyage coïncide avec la visite de Donald Trump à Riyad. Le 22, il rencontre brièvement Donald Trump.

Rachid Dostum se rend en Turquie " pour raisons de santé ». Beaucoup pensent qu'il y a été contraint.

- Le 22 : 6 tonnes de nitrate d'ammonium (utilisé pour fabriquer des explosifs) sont saisis dans un bus pakistanais dans la pro- 
vince de Nangarhâr.

Six Tâlebân du Conseil de Quetta sont arrêtés dans la province de Kandahar.

Le Centre de justice anticorruption condamne un responsable de l'Afghan United Bank à 15 ans de prison et une amende de 3 millions de dollars pour détournement de fonds.

- Le 24 : Les partis du Conseil national suprême du Djihad formé il y a deux ans annoncent avoir retiré leur soutien au gouvernement, car il n’a pas respecté ses promesses.

Hâmed Karzaï rencontre dans son bureau l'ambassadeur de Russie en Afghanistan.

Dans son premier budget annuel, D. Trump propose 5 milliards de dollars pour renforcer les forces de sécurité afghanes.

- Le 25 : La Mission d'assistance des Nations unies en Afghanistan dit avoir reçu des familles de victimes de la guerre une requête en faveur d'un procès du Hezb-e Islami, pour participation à des crimes.

Onze hauts fonctionnaires de la Défense sont condamnés à des peines de prison (de un an et demi à deux ans) par une cour militaire suite à l'attaque récente de l'hôpital militaire de Kaboul.

- Le 26 : L’Afghanistan et le Tadjikistan signent un sixième accord de coopération économique et commerciale. Suite à la fermeture des frontières avec le Pakistan, les relations commerciales entre les deux pays auraient beaucoup augmenté ces derniers

Le 31 : A Kaboul, un attentat au camion piégé tue 90 personnes et fait 380 blessés près du quartier diplomatique. Les victimes sont principalement des civils. L'explosion provoque d'énormes dégâts à la ronde. Le porte-parole des Tâlebân nie toute responsabilité. Des habitants de Kaboul reprochent au gouvernemen d’être défaillant au plan de la sécurité.

mois, faisant de l'Afghanistan l'un des principaux partenaires commerciaux du Tadjikistan. Au premier trimestre 2017, le Tadjikistan a exporté 74 millions de dollars en marchandises vers l'Afghanistan, dont 11 millions de ciment.

Les États-Unis approuvent un contrat de 76,7 millions de dollars en hélicoptères pour la Force aérienne afghane.

- Le 27 : Des combattants iraniens affiliés à Daech sont arrêtés dans le district de Haska Mina (Nangarhâr).

Le Pakistan annonce, à l'occasion du début du ramadan, la réouverture de sa frontière avec l'Afghanistan à Chaman.

- Le 28 : Achraf Ghani suspend les maires de Hérat et de Djalâlâbâd, suite à des plaintes répétées de leurs habitants.

L'Afghanistan, la Chine et le Pakistan tiennent leur premier round de dialogue trilatéral à Pékin afin de favoriser la paix.

Les représentants de douze pays, dont

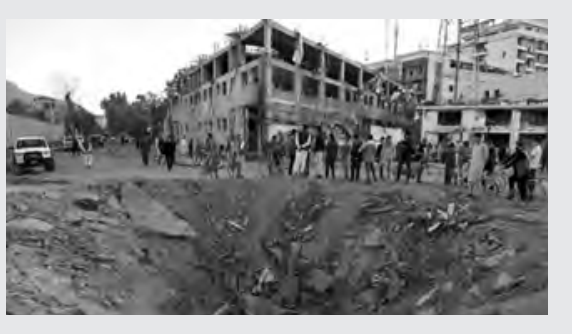

l'Afghanistan, se réunissent en Chine pour la présentation du projet chinois de la nouvelle route de la soie.

- Le 29 : 24 officiers sont licenciés suite à l'attaque le mois dernier par les Tâlebân contre la base de Mazar-e Charif.

L'Union européenne annonce 42,5 millions d'euros pour les personnes déplacées et les réfugiés afghans en Iran et au Pakistan.

- Le 30 : Le ministère russe des Affaires étrangères interroge les États-Unis et l'OTAN sur l'appartenance des avions non-identifiés qui fourniraient des armes et d'autres matériels militaires aux combattants de Daech dans la province de Djaozdjân. Des soupçons ont été par ailleurs émis sur la présence d'hélicoptères russes dans des zones tenues par les Tâlebân.

(Source principale : Bulletin du CEREDAF)

\section{DERNIERES PUBLICATIONS}

\section{Articles}

- « La France vue par les réfugiés », par Grégoire Biseau, Martin Colombet et Hans Lucas, in Libération 06/03/17

- « A Kaboul, un cinéma «familial», bol d'air pour les femmes », AFP/La Croix 16/03/17

- « L’expulsion des Afghans, une polémique qui enfle en Allemagne » par Delphine Nerbollier, La Croix 25/04/2017

- « États-Unis : l’émouvante histoire des amants de Bamiyan, menacés de mort en Afghanistan ", par Maurin Picard, Le Figaro 25/04/2017

- « Le Deuxième Sexe » fait école à Kaboul », par Julien Bouissou, $M$ le magazine du Monde, 28/04

- « Dans les salons de beauté de Kaboul, toutes les audaces sont permises », par AFP/La Croix 05/05

- « Afghanistan : dernier zoom sur Mes Aynak avant disparition », par Florence Evin et Julien Bouissou, Le Monde 05/05 - « Logiques transfrontalières et salafisme globalisé : l'Etat islamique en Afghanistan ", par Adam Baczko, Gilles Dorron- soro, Presses de Sciences Po (P.F.N.S.P.), « Critique internationale »2017/1 N 74 pages 137 à 152

- « En Afghanistan, les trésors révélés de Mes Aynak », par Florence Evin et Julien Bouissou, Le Monde 11/05

- «Avec l'État islamique nous oublions le vrai danger : al-Qaida ", par Michael Kugelman, traduit par Antoine Bourguilleau, Slate.fr 10/05

- « Ces 4 expatriées défient le chaos quotidien à Kaboul », reportage de Solène Chalvon, Grazia 30/05

- " Mémoire de Bamiyan ", 11 épisodes. Webdocs. parus dans Courrier International du 10/03 au 20/05 2017.

\section{Revues}

- Afghanistan Info, nº8, avril 2017. (dernier numéro de cette revue publiée par le Comité suisse de soutien au peuple afghan) : The study of Afghanistan : Pathways followed, opportunities ahead par William Maley, Myth and reality of the ismalic State in Khorasan par An tonio Giustozzi, Zum phänomen des sog. Islamischen staates in Afghanistan par Katja Mielke, Kabul remembered par Nancy Hatch-Dupree, Géo-nostalgie de l'Afghanistan par Olivier Roy, Une antropologie imaginaire par Gilles Dorronsoro, Homo itinerans par Alessandro Monsutti, Instantatnés sur des écoles de la province de Bamiyan par Etienne Gille.

- Afghanistan Nytt, $n^{\circ} 1,2017$, édité par le Comité suédois pour l'Afghanistan, en suédois. 28p

\section{Livres}

- Roxanne, roman d'Assem Akram, éd. Balland (Avril 2017), 228p., ISBN : 9782940556557

- Al-Qaida in Afghanistan, d'Anne Stenersen, Cambridge University Press (Mars 2017), 300p., ISBN : 978-1107427761 - Sur les routes d'Afghanistan, de PierreJean Laforêt, éd. Aéropage, 2016, 148p. -Visages de guerre - Les gueules cassées de la Grande Guerre à l'Afghanistan, de Sophie Delaporte, éd. Belin (mars 2017), 368p., ISBN: 978-2701190884

- L’Afghanistan, une aventure géopolitique 
française, de Stéphane Charles Natale, éd. L'Harmattan (mai 2017), 300p., ISBN : 978-2-343-11670-9

- L'Infanterie mécanisée allemande au combat en Afghanistan, de Marcel Bohnert et Andy Neumann, Books on Demand (Avril 2017), 104p., ISBN : 9783743151994

- Afghanistan: Preserving its Historic Heritage: The AGA Khan Historic Cities Programme, sous la direction de Philip Jodidio, Prestel Pub. (Mai 2017), 368p., ISBN : 978-3791356433

- The Hazaras and the Afghan State: Rebellion, Exclusion and the Struggle for Recognition, de Niamatullah Ibrahimi, C Hurst \& Co Publishers Ltd (Mai 2017), 224p., ISBN : 978-1849047074

- The Afghanistan Poppy Eradication Campaign: Accounts from the Black Hawk Counter-narcotics Infantry Kandak Team in Helmand Province, de Harry Spiller, McFarland \& Co Inc (Mai 2017), 129p., ISBN : 978-1476668642

- The Taliban Reader - War, Islam and Politics in their Own Words, d'Alex Strick Van Linschoten et Felix Kuehn, C Hurst \& Co Publishers Ltd. (Mai 2017, 320p., ISBN : 9781849048095

Les dates de parution des livres ne sont données qu'à titre indicatif. S'informer auprès des éditeurs. Pour une bibliographie plus complète, consulter le Bulletin du CEREDAF.

\section{Notes de lectures}

\section{La rupture de l'exil}

Une enfance heureuse dans un milieu éduqué et ouvert à Kaboul et à Estalef où la famille possède une propriété. Des études au lycée Malalaï, puis l'enseignement comme professeur dans le même établissement et ensuite comme enseignante au département de français de l'Université de Kaboul. La vie de Nasrine Nabiyâr aurait pu être un long fleuve tranquille. Mais survient en 1978 la « révolution ». Nasrine et son mari tiennent quelques années, mais les enfants ayant été traumatisés par la situation de violence et les chutes de roquettes, Nasrine part en Inde début 1989 avec seulement ses deux plus jeunes fils. La famille sera réunie une année plus tard en France où Nasrine avait fait plusieurs séjours d'étude. Une vie nouvelle commence à Rennes dans un quartier cosmopolite.

Nasrine Nabiyâr n'oublie pas son pays et dès 1998 crée l'association Malalaï pour venir en aide à des familles réfugiées et à des professeurs. Dès que la paix revient, elle se rend en Afghanistan. Voyant les besoins d'éducation à Estalef, elle conçoit le projet d'un collège pour filles. La première pierre est posée le 16 septembre 2002 pour ce collège appelé « Atéfa-e Chahid » en mémoire d'une fillette morte sous un bombardement soviétique. Nasrine fait preuve de beaucoup de ténacité en France pour trouver les financements et en Afghanistan pour obtenir les autorisations. Rapidement le collège voit le jour ; il sera agrandi par la suite.

Dans le livre qu'elle vient de publier, on suit pas à pas Nasrine de son enfance à aujourd'hui : elle se sent à l'aise dans les deux cultures, deux modes de vie. Elle a à cœur de venir en aide aux femmes afghanes. On lit avec beaucoup d'émotion les événements qu'elle raconte tant à Estalef qu'à Kaboul. Moments tragiques vécus par les habitants : bombardements soviétiques, disparitions, action des Tâlebân, exil des uns ou des autres à l'intérieur du pays ou ailleurs ; mais aussi joies : accueil chaleureux des personnes rencontrées, beauté des paysages, mode de vie afghan. L'auteure est très émue chaque fois qu'elle retourne au pays et nous fait partager ses peines et son bonheur. On ne peut qu'admirer sa détermination pour mener à bien ses projets éducatifs. Elle effectue aussi des missions d'enseignement du français à l'Université de Kaboul et accompagne le projet d'une clinique mobile initié $\mathrm{p}$ ar « Mères pour la paix ». Son livre est une leçon. Comment transformer la douleur de l'exil en dynamisme au service de son pays. Marie-Josèphe GILLE

- L'exil, la rupture, départ à contre-cœur, Nasrine Nabiyar, 226 p, 15 euros plus 4,3 euros de frais de port. Contacter nnabiyar@ hotmail.fr.

\section{Roxanne}

Bien connu des amis français de l'Afghanistan, Assem est le fils aîné de Mohamed Akram qui fut ambassadeur dans notre pays. Docteur en Histoire, auteur d'une « Histoire de la guerre en Afghanistan » et d'un roman "Ocre fatal », tous deux parus chez Balland, c'est des EtatsUnis, où il réside depuis une vingtaine d'années que l'auteur a écrit en français cette chronique de la vie quotidienne d'exilés afghans aux USA.

Rédigé par petites touches, l'ouvrage est composé de textes enchâssés qui tournent autour du personnage principal : cette Rushana devenu Roxanne, quinquagénaire au verbe cru, dépeinte par un auteur qui n'est pas tendre avec elle et beaucoup de ceux qui l'entourent, famille ou amis. Une belle galerie de personnages qui sont autant de représentations des acteurs de nos sociétés, de leurs faiblesses comme de leurs noblesses. Mais la particularité de cette comédie humaine, c'est qu'elle est constituée d'américano-afghans, et que pour vivre

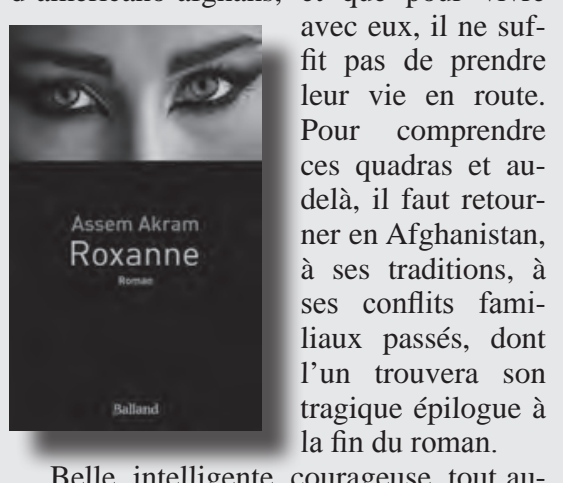

Belle, intelligente, courageuse, tout autant que colérique, envieuse, autoritaire, Roxanne navigue à vue entre Aziz, un mari faible et alcoolique, des filles rebelles, des amies snobes, de braves types comme " Chékam »; entre la vie américaine, les traditions afghanes ; entre deux cultures, deux niveaux de vie, des souvenirs et souvent un avenir incertain.

Le roman réaliste d'Assem Akram est écrit dans une langue française que l'éditeur n'a pas souhaité corriger, polir, pour nous le livrer à l'état brut, ce qui donne quelques coquilles et quelques trouvailles, mais il faut le prendre comme le charme de l'écriture d'un homme qui après vingt ans témoigne de son amour de la langue française.

\section{Jack CHABOUD}

- Roxanne, par Assem Akram. 226 pages. Editions Balland. $18 €$

\section{Suspendue à un fil}

Médecin psychiatre et pédiatre, Massouda Jalal a dû fuir son pays à la prise du pouvoir par les Tâlebân. Elle a depuis entrepris un combat pour les femmes afghanes au travers de sa « Fondation Jalal » et de ses prises de position politiques et féministes, qui l'ont conduite à se présenter à une élection présidentielle et à occuper une fonction ministérielle qui lui a permis de faire adopter un « Plan d'action nationale pour les femmes d'Afghanistan » et un décret sur « L'élimination de la violence contre les femmes afghanes ».

Dans cet ouvrage, elle retrace un historique de la position des femmes de son pays, en rappelant les rares moments où elles purent sortir de leur véritable asservissement, et en annonçant l'épouvantable sort qui les attend si les Tâlebân, comme c'est à craindre, reviennent au pouvoir. 


\section{DERNIERES PUBLICATIONS}

Après avoir décrit la lutte inégale des puissants (clans, religieux conservateurs...) contre les tentatives de réformateurs et de la communauté internationale, pour aider les Afghanes à échapper aux traditions machistes qui les ramènent parfois à la position de bêtes, quand ce n'est pas à celle d'objets inanimés. Et pourtant, souvent au péril de leurs vies, des femmes afghanes réussissent malgré tout à franchir les obstacles avec un

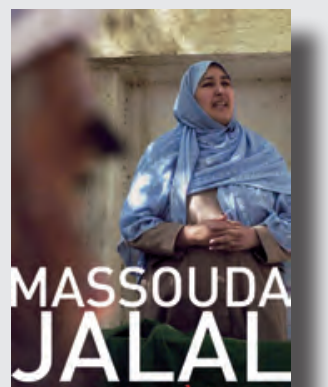
grand courage, à fréquenter les écoles, occuper des métiers aussi nouveaux pour elles que l'armée ou la police. Face à ces succès, l'ouvrage de Massouda Jalal comprend de nombreuses notes relatant les abominables circonstances de viols et meurtres de femmes afghanes. Il permet enfin au lecteur de saisir l'étendue de la misère des ces femmes battues, violées, tuées, au nom de traditions archaïques et barbares, dans leurs propres familles comme dans la société, on songe au « Syngué sabour » d’Atiq Rahimi ou au « Mille soleils splendides » de Khaled Hosseini. Elle dénonce les arguments mensongers ou fanatiques qui justifient le martyr des femmes afghanes, elle en appelle à la communauté internationale et aux progressistes de son pays pour mettre fin à la corruption, pour protéger les femmes devant un retour des Tâlebân, pour tenter d'instaurer une paix durable dans son pays. Que l'avenir puisse lui donner raison. Jack CHABOUD

- "Suspendue à un fil. La longue lutte pour les femmes afghanes ». 260 pages. Massouda Jalal. Ed. Balland. $18 €$

\section{Les forces françaises en Afghanistan}

L'engagement des forces françaises en Afghanistan a suscité de nombreux ouvrages (témoignages, stratégie, géopolitique). Près de cinq ans après leur retrait, cette floraison se poursuit. On retiendra deux livres parus récemment : « Médecin en Afghanistan-journal de marche d'un médecin militaire ordinaire en opération extérieure " par Etienne Philippon aux Editions Lavauzelle et « 1929 jours » par Nicolas Mingasson aux Editions Les belles lettres.

Rappelant en avant-propos que cinq infirmiers et brancardiers secouristes fran-

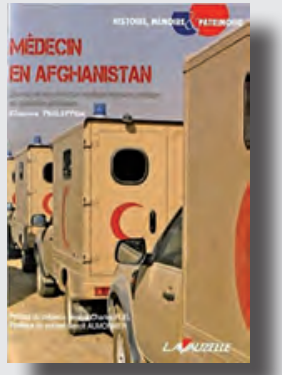

çais sont morts en Afghanistan, le médecin chef Philippon raconte avec humilité et sincérité sa mission : six mois en OMLT (operationnal mentoring and liaison team) au sein d'un kandak ${ }^{1}$ de l'armée afghane dans le district de Surobi. Une dualité apparaît entre les conditions de vie parfois un peu décalées des éléments français (séquence de préparation dans un village afghan reconstitué en Bavière, BFMTV et France 24 en continu, clips musicaux d'une chaine polonaise « pour les légionnaires », colis de charcuterie, liaison Skype quasi quotidienne) et celles nettement plus précaires des Afghans (hygiène limite) même si elles se rejoignent en opération ou dans la menace des IED (engins explosifs improvisés). Le parcours du chef afghan du kandak a épousé les sinuosités de l'histoire récente de son pays : pachtoune de Djalalabad, il a combattu les modjahedin jusqu'aux derniers jours de l'armée de Nadjibullah ; sous le régime tâleb, il devient paysan et vendeur de vêtements ; en 2007 , il rempile au sein de l'armée avec un grade de lieutenant-colonel et un Tadjik d'Herat comme garde du corps.

Si le "mentoring ${ }^{2}$ ne convainc pas pleinement l'auteur (10\% de satisfaction pour $90 \%$ de déception), il relève le courage et la discipline des soldats afghans qui fonctionnent surtout sur leurs ressorts traditionnels, au prix de quelques rigidités (caporalisme), arrangements (une corruption « culturelle ») et dérives (la compagnie compte nombre de consommateurs de drogue).

Dans la beauté des paysages de la Kapisa, le médecin chef se montre désireux de contacts et de signes de proximité avec ses « mentorés » : partage des fruits comme les pommes et les grenades " fruit sacré dans le coran et dont il est mal vu de faire tomber un grain ", lieu de prières avec ses quelques tapis et fleurs entourés de barbelés, des relations fondées sur la confiance plus que sur l'obéissance, le goût pour la blague des Afghans, leur côté mauvais perdant, leur réticence à se déshabiller pour des consultations médicales.

" Les Afghans sont des noceurs, des poètes, des chanteurs, des bons vivants. Est-ce la raison pour laquelle les Français s'entendent si bien avec eux ? " s'interroge l'auteur, attaché au succès de sa mission mais sans trop d'illusion sur sa portée.

Tout autre est le propos de Nicolas Mingasson. Journaliste, il s'est beaucoup investi dans l'engagement des forces françaises (couverture de presse pendant six mois en 2010 en Kapisa, cosignature avec Christophe Tran Van Can en 2011 du « Journal

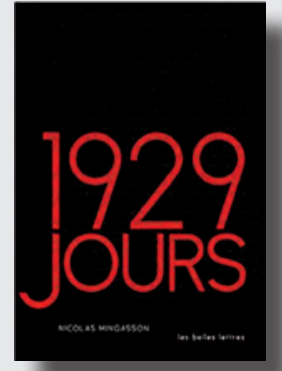

d'un soldat français en Afghanistan » aux Editions Plon). Dans « 1929 jours " - décompte effectué par la mère d'un soldat tué il aborde le douloureux chapitre des victimes de ce conflit. « De 2001 à 2011, l'on estime qu'environ 70000 soldats français ont servi en Afghanistan. Parmi ceux-ci, 90 sont morts (au combat, d'accident, de causes naturelles ou par suicide - trois ou quatre suicides sont avérés selon l'auteur) dont 85 officiellement « morts pour la France » et 700 autres sont blessés ».

Pour « l’Afgha, cette mission qu'il ne fallait pas rater » comme on disait dans les unités, il y aura un avant et un après Uzbin (embuscade faisant 10 morts en août 2008), l’année 2011 se révélant particulièrement meurtrière.

Pendant deux ans, l'auteur a accompagné les familles des victimes, parents, compagne, frères et sœurs, enfants, dans leur deuil. Un long cheminement qui passe par l'évocation du départ en opération extérieure, dans l'exaltation, le silence et la peur, le retour des missions lointaines et incertaines, l'annonce de la disparition entre sidération et déni, colère et douleur (« Tout explose, il y a un clash qui déchire votre vie en un avant et un après . vous êtes là, physiquement, mais votre esprit part ailleurs »), la reconnaissance de la Nation dans des cérémonies officielles vécues parfois avec distance. Et, au bout, ce décompte des « 1929 jours » qui ne finira jamais. Une grande dignité nourrit de part et d'autre ce témoignage.

L’Afghanistan surgit parfois au détour des pages : l'attrait de la mission et de ses risques dans un pays « béni » pour les uns, plein d'incertitude et d'angoisse pour les autres comme le relève Nicolas Mingasson, les « chouras » ces «missions de merde » compte tenu de la taille du périmètre à sécuriser, le déficit d'information pour les familles sur le contenu et le sens de cet engagement, les Tâlebân à qui on va faire « bouffer la poussière ».

Une lecture poignante où « l'Afgha » apparaît si proche et si lointain. Pour toujours. Régis KOETSCHET

- Médecin en Afghanistan-journal de marche d'un médecin militaire ordinaire en opération extérieure, par Etienne Philippon aux Editions Lavauzelle

- 1929 jours par Nicolas Mingasson aux Editions Les Belles Lettres.

1- Mot d'origine pachto utilisé dans l'armée afghane pour désigner un bataillon.

2- Relation de conseilleur (le mentor) à conseillé. 


\section{Le rêve d'Alexandre}

Jean-Pierre Perrin a repris le chemin de l'Afghanistan. Après les Kamaz (camion russe) brinquebalants, les ânes de la Chamali et l'escalade de nuit des cols du Pandjchir des « Jours de poussière » de la résistance à l'intervention soviétique (la Table Ronde 2002) , l'écrivain-journaliste - et ancien des missions humanitaires d'AFRANE - s'est, cette fois, enrôlé dans l'armée d'Alexandre, l'Iskander Kabir des Afghans. Près de 50 000 hommes dont 5000 cavaliers en compagnie d'une nuée de bâtisseurs, savants, lettrés, musiciens et prostituées aux tentes ornées de bouquets de myrrhe.

Le mépris du danger, l'hubris et la passion sont, au quotidien, du voyage. Après avoir défait Darius et s'être lancé à la poursuite de l'usurpateur Bessos, le Macédonien entre en Afghanistan. II y fonde les Alexandrie d'Arie (Herat), d'Arachosie (Kandahar) et du Caucase (Bagram). A Balkh, appelée à devenir la rivale de Babylone, la « mère de toutes les villes » (oum el balad), il épouse Roxane, la « resplendissante ».

Avec Alexandre et ses héritiers s'ouvre une histoire afghane à l'imaginaire généreux et dont témoigne « le rêve si pacifique du Gandhara ». " La suprême sagesse par la suprême beauté » écrira Malraux.

Mais l'auteur, familier des théâtres de guerre en Orient, sait que la géographie et l'histoire sont souvent injustes et cruelles. Elles le seront pour l'Afghanistan. Si Alexandre emmène sa troupe jusqu'aux rives de l'Indus, c'est en ayant à l'esprit la centralité de l'Afghanistan. Avec Aristote, il sait que des cimes de l'Hindou Kouch on peut découvrir le reste du monde et que c'est autour de ce pivot que l'on peut rêver l'Eurasie - et pour d'autres, œuvrer avec acharnement à la destruction de ce carrefour. Devant l'auteur, le commandant Massoud, «l'Afghan qui a gagné la guerre froide » pour le Wall Street Journal, dessinera ce planétaire jeu de l'oie géopolitique dont Alexandre aura occupé les premières cases.

Enivré comme tant d'autres après lui par l'air pur des montagnes afghanes, Iskander enferme sa traque de Bessos/Ben Laden dans une vengeance illimitée, confortant la malédiction afghane de la violence. L'historien Gabriel Martinez-Gros y voit désormais un désir de guerre contre l'histoire, « un djihad mené contre le passé ».

D'une plume érudite et brillante, à la fois bienveillante et sans concession, stimulante pour le lecteur, l'auteur remonte « les couloirs du temps » afghan, de « 330 avant J.-C. à 2016 », aux côtés des hordes turcomongoles qui s'annoncent par leur odeur (« II s'y mêle l'urine forte des coursiers trempés d'écume, la sueur noire imprégnée de sang séché des cavaliers, le suri du lait caillé qui les nourrit et les relents pestilentiels des charniers qu'ils trainent avec eux »), des acteurs décalés du « Grand Jeu », des tribus pachtounes et des djihadistes « sans frontières » tels le Fakir d'Ipi ou Djalal ud-din Haqqani. On y croise des "figures » d'Alexander Burnes à Dominique Vergos - aimantées, comme Alexandre, par ce pays, ses «confins » et ses tourments.

La mémoire collective en cette région du monde reste imprégnée par l'expédition d'Alexandre le Grand. Les officiers de l'OTAN s'inspirent du stratège, l'historien Worthington en fait le promoteur du « nation building ».

Jean-Pierre Perrin veut, pour sa part, retenir la poésie et le rêve qui entourent cette aventure sachant qu'ils constituent les vraies « clefs » de l'Afghanistan. Plutarque a rapporté qu'Alexandre lisait chaque soir une page de l'lliade d'Homère ou des Chroniques du « barbarophile » Hérodote. Le poète persan Nizâmi mettra en vers l'épopée du Macédonien. Quant au « rêve eurasien » fondateur, illustré par le geste d'Alexandre se dépouillant de son manteau pour y couvrir le cadavre de Darius assassiné, Nicolas Bouvier le qualifie de " si beau, perspicace, intemporel et généreux ». Mais la dite « communauté internationale » sait-elle aujourd'hui encore rêver?

Le livre vient d'obtenir le prix Joseph Kessel du Festival Étonnants Voyageurs.

A signaler également le beau livre publié à l'automne par Jean-Pierre Perrin (Menaces sur la mémoire de l'humanité - Editions Hoëbeke) et dont un chapitre est consacré à « l'Afghanistan mutilé ».

\section{Régis KOETSCHET}

- Le djihad contre le rêve d'Alexandre, de Jean-Pierre Perrin, Le Seuil 